\title{
Abstracts for the Human Genetics Society of Australasia Virtual Conference 24-25 November 2020
}

\section{Orals}

\section{Oral 1}

'We Just Wanted to Get it Done': The Parent Experience of Rapid Acute Care Genomics

Fiona Lynch ${ }^{1,2,3}$, Belinda McClaren ${ }^{1,2,3}$, Amy Nisselle ${ }^{1,2,3}$ and Clara Gaff ${ }^{1,2,3}$

${ }^{1}$ Australian Genomics Health Alliance, Melbourne, Australia, ${ }^{2}$ Genomics in Society, Murdoch Children's Research Institute, Melbourne, Australia and ${ }^{3}$ The University of Melbourne, Melbourne, Australia

Genomic technologies are showing great promise in neonatal and pediatric acute care, particularly as ultra-rapid genomic sequencing (rGS) can now facilitate test results within days for critically unwell children. Novel genetic counseling issues regarding rGS have recently been reported; it is vital that we also understand parents' experiences to inform the delivery of genetic counseling in this setting. Semistructured interviews were therefore conducted with 30 parents (19 mothers) of 20 children who had rGS while in acute care (2019-20). Audio-recorded interviews were transcribed, and data analyzed using reflexive thematic analysis. Parents received either diagnostic $(n=9,45 \%)$, negative $(n=7,35 \%)$, or uncertain $(n=$ $4,20 \%)$ results for their child. Six children (30\%) were deceased at the time of interview. Parents appreciated how fast the test was performed, and felt lucky to be offered special access to 'exciting', 'expensive' and 'cutting-edge' research. Parents readily consented to genomic testing, with seemingly little consideration for other implications of the test, in the hope it may provide information to guide treatment or understand prognosis. Although participants recognized the need for a fast turnaround, some expressed feeling rushed to consent to rGS. Further, whilst parents found information about genomic testing complex and overwhelming, they recalled receiving informational and emotional support from a variety of health professionals involved in their child's care. While there are clear clinical benefits to rGS, this data raises questions about whether a rapid turnaround is right for all families. Regardless, additional genetic counseling considerations are needed to support families impacted by this technology.
Oral 2

Mutations in the Exocyst Component EXOC2 Cause Severe Defects in Human Brain Development

Nicole Van Bergen ${ }^{1,2}$, Muktar Ahmed ${ }^{3}$, Felicity Collins ${ }^{4,5}$, Mark Cowley ${ }^{6,7,8}$, Annalisa Vetro ${ }^{9}$, Russell Dale ${ }^{10,11}$, Daniella Hock ${ }^{12}$, Christian De Caestecker ${ }^{3}$, Minal Menezes ${ }^{13}$, Sean Massey ${ }^{1}$, Gladys Ho ${ }^{4}$, Tiziana Pisano ${ }^{9}$, Seana Glover ${ }^{1,2}$, Jovanka Gusman $^{1,2}$, David Stroud ${ }^{12}$, Marcel Dinger ${ }^{6,14}$, Renzo Guerrini ${ }^{9}$, Ian Macara ${ }^{3}$ and John Christodoulou ${ }^{1,2,13,15}$

${ }^{1}$ Murdoch Childrens Research Institute, Melbourne, Australia, ${ }^{2}$ Department of Paediatrics, University of Melbourne, Melbourne, Australia, ${ }^{3}$ Department of Cell and Developmental Biology, Vanderbilt University School of Medicine, Nashville, USA, ${ }^{4}$ Western Sydney Genetics Program, Children's Hospital at Westmead, Sydney, Australia, ${ }^{5}$ Medical Genomics Department, Royal Prince Alfred Hospital, Sydney, Australia, ${ }^{6}$ Kinghorn Centre for Clinical Genomics, Garvan Institute of Medical Research, Sydney, Australia, ${ }^{7}$ St Vincent's Clinical School, UNSW Sydney, Sydney, Australia, ${ }^{8}$ Children's Cancer Institute, Kensington, Australia, ${ }^{9}$ Pediatric Neurology, Neurogenetics and Neurobiology Unit and Laboratories, Meyer Children's Hospital, University of Florence, Florence, Italy, ${ }^{10}$ Department of Paediatric Neurology, The Children's Hospital at Westmead, Sydney, Australia, ${ }^{11}$ Kids Neuroscience Centre, Faculty of Medicine and Health, The University of Sydney, Sydney, Australia,

${ }^{12}$ Department of Biochemistry and Molecular Biology and The Bio21 Molecular Science and Biotechnology Institute, The University of Melbourne, Melbourne, Australia, ${ }^{13}$ Kids Research, The Children's Hospital at Westmead, Sydney, Australia, ${ }^{14}$ School of Biotechnology and Biomolecular Sciences, University of New South Wales, Kensington Campus, Sydney, Australia and ${ }^{15}$ Victorian Clinical Genetics Services, Royal Children's Hospital, Sydney, Australia

Background: An accurate genetic diagnosis for neurodevelopmental disorders has great potential to improve patient care and quality of life. Aims: We undertook a gene discovery approach in individuals with severe developmental delay, dysmorphism, and brain abnormalities, poor motor skills and variable epilepsy. Methods: Whole exome sequencing identified candidate genes. Candidate genes were validated in patient fibroblasts by western blot, qPCR, vesicular trafficking assays, lentiviral gene rescue and cilia morphology. Results: Here, for the first time, we report pathogenic variants in an exocyst subunit, EXOC2 (Sec5). Family 1 had two offspring with a 
homozygous truncating variant that leads to nonsense-mediated decay of EXOC2 transcript, and undetectable levels of EXOC2 protein. Fibroblasts from an affected individual had a severe reduction in tsVSV-GFP exocytosis and VAMP-pHluorin vesicle fusion (reduced by $\sim 50 \%)$. The affected individual from Family 2 had compound heterozygous mutations, a milder clinical phenotype and fibroblasts had reduced tsVSV-GFP exocytosis (reduced by $\sim 40 \%$ ). Fibroblasts from both patients showed defective Arl13b localization to the primary cilium (reduced by $\sim 30 \%$ ). Discussion/Conclusion: The exocyst is an octameric protein complex essential for membrane transport, tethering and fusion of post-Golgi vesicles at the plasma membrane, prior to membrane fusion. The exocyst functions in dendritic arborization and axon elongation in neurons. The discovery of mutations that partially disable exocyst function provides valuable insight into exocyst complex in neural development. Since EXOC2 and other exocyst complex subunits are critical to neuronal function, our findings suggest that EXOC2 variants are the cause of the patients' neurological disorders.

\section{Oral 3 \\ Health Professionals' Views and Experiences Following Commencement of the Genetics and Life Insurance Moratorium}

Jane Tiller ${ }^{1,2,3}$, Louise Keogh ${ }^{4}$, Aideen Mclnerney-Leo ${ }^{5}$, Andrea Belcher ${ }^{6,7}$, Kristine Barlow-Stewart ${ }^{8}$, Tiffany Boughtwood ${ }^{3,7}$, Martin Delatycki ${ }^{2,3}$, Ingrid Winship ${ }^{9,10}$, Margaret Otlowski ${ }^{11}$ and Paul Lacaze ${ }^{1}$

${ }^{1}$ School of Public Health and Preventive Medicine, Monash University, Melbourne, Australia, ${ }^{2}$ Victorian Clinical Genetics Services, Melbourne, Australia, ${ }^{3}$ Murdoch Children's Research Institute, Melbourne, Australia, ${ }^{4}$ Centre for Health Equity, Melbourne School of Population and Global Health, The University of Melbourne, Melbourne, Australia, ${ }^{5}$ The University of Queensland Diamantina Institute, University of Queensland, Brisbane, Australia, ${ }^{6}$ Faculty of Medicine, University of Queensland, Brisbane, Australia, ${ }^{7}$ Australian Genomics Health Alliance, Melbourne, Australia, ${ }^{8}$ Sydney Medical School, University of Sydney, Sydney, Australia, ${ }^{9}$ Department of Medicine, The University of Melbourne, Melbourne, Australia, ${ }^{10} \mathrm{Genomic}$ Medicine and Family Cancer Clinic, Royal Melbourne Hospital, Melbourne, Australia and ${ }^{11}$ University of Tasmania, Hobart, Australia

Background: The industry self-regulated moratorium on genetics and life insurance, which limits life insurers' use of genetic test results in underwriting, commenced in July 2019. Methods: We surveyed health professionals (HPs) who discuss genetic testing with patients about levels of training and understanding regarding the moratorium; experiences of patient behavior and attitudes following its commencement; and views regarding regulation. Results: 166 eligible HPs participated in the online survey. $86 \%(n=142 / 166)$ of HPs were aware of the moratorium, but $<50 \%(n=70 / 166)$ had attended training or information sessions about it. Only $16 \%(n=23 / 166)$ answered all knowledge questions correctly; however, 69\% $(n=$ $114 / 166$ ) believed they have sufficient knowledge to advise patients. There was some reported reduction in patients delaying or refusing testing due to insurance concerns, but $49 \%(n=71 / 144)$ and $42 \%(n$ $=61 / 144$ ) respectively of HPs disagreed that patients are less confused, and more willing to have testing, than before the moratorium. $76 \%(n=110 / 144)$ of HPs felt the moratorium resolved some concerns about insurance discrimination, but $88 \%$ (127/144) still have concerns about insurance discrimination following its introduction. Almost half ( $n=73 / 149)$ of the HPs reported being somewhat or very dissatisfied with the moratorium as a solution to genetic discrimination. 95\% $(n=141 / 149)$ believe that an agreement between the government and insurance industry regarding the moratorium is required. $93 \%(n=139 / 149)$ also believe that the government should introduce legislation to regulate the use of genetic test results in life insurance underwriting. Conclusion: Health professionals report that while the moratorium has assuaged some concerns, it does not sufficiently address concerns with genetic discrimination in life insurance.

\section{Oral 4 \\ Diagnostic Outcomes of the Australian Genomics Brain Malformations Flagship}

Daniz Kooshavar ${ }^{1,2}$, Kate Riney ${ }^{3}$, Christopher Barnett ${ }^{4}$, Jonathon Silberstein ${ }^{5}$, Rani Sachdev $^{6}$, Shekeeb S. Mohammad ${ }^{7}$, Kathryn North ${ }^{1,8}$, Tiffany Boughtwood ${ }^{1,8}$, Michael Fahey ${ }^{9}$, Ingrid Scheffer ${ }^{10}$, Nicholas Smith ${ }^{11}$, Tyson Ware ${ }^{12}$, Paul J Lockhart ${ }^{1,2}$ and Richard J. Leventer ${ }^{1,2,13}$

${ }^{1}$ Murdoch Children's Research Institute, Melbourne, Australia, ${ }^{2}$ Department of Paediatrics, The University of Melbourne, Melbourne, Australia, ${ }^{3}$ Queensland Children's Hospital, University of Queensland, Brisbane, Australia, 'SA Clinical Genetics Service, Women's and Children's Hospital, Adelaide, Australia, ${ }^{5}$ Department of Neurology, Princess Margaret Hospital, Perth, Australia, ${ }^{6}$ Department of Medical Genetics, Sydney Children's Hospital, Sydney, Australia, ${ }^{7}$ Department of Neurology, Westmead Hospital, Sydney, Australia, ${ }^{8}$ Australian Genomics Health Alliance, Melbourne, Australia, ${ }^{9}$ Monash Medical Centre, Monash University, Melbourne, Australia, ${ }^{10} \mathrm{Florey}$ Institute, The University of Melbourne, Melbourne, Australia, ${ }^{11}$ Department of Neurology and Clinical Neurophysiology, Women's and Children's Hospital, Adelaide, Australia, ${ }^{12}$ The Royal Hobart Hospital, University of Tasmania, Hobart, Australia and ${ }^{13}$ The Royal Children's Hospital, Melbourne, Australia

Malformations of brain development (MBD) represent a large group of conditions resulting from disturbances in brain formation during pregnancy While individually rare, collectively MBD are a major cause of disability, cerebral palsy and epilepsy. This cohort project aimed to determine the genetic diagnostic yield in MBD patients using singleton clinical whole exome sequencing (WES). We performed a nation-wide multi-centre cohort study as part of the Australian Genomics Health Alliance. Patients were selected based on stringent inclusion criteria regarding age, clinical features, and prior genetic testing. Pathogenic/likely pathogenic variants identified in clinically relevant genes were reported according to the American College of Medical Genetics standards and guidelines. Genetic causes were identified in 38/102 (37.2\%) patients: $8 / 38$ (21\%) had Pathogenic/likely pathogenic variants in TUB1A1; $2 / 38(5.3 \%)$ in DCX, 2/38 (5.3\%) in TUBB2B, 2/38 (5.3\%) in DYNC1H1, 2/38 (5.3\%) in FLNA, $2 / 38$ (5.3\%) in FOXG1; and the remaining 20/38 (52.6\%) had Pathogenic/likely pathogenic variants(s) in one of the following genes: ACTB, AHI1, CASK, CEP85L, CHMP1A, COL4A1, DCHS1, DEPDC5, DHCR7, KIF1A, KIF1BP, NIPBL, NSDHL, PIGG, POMGNT1, SON, TMEM237, TSEN54, TUBB2A, and $Z B T B 18$. This study reached a genetic diagnostic rate of $37.2 \%$, which is consistent with previous smaller studies. We confirmed that using WES for genetic diagnosis of MBD is an efficient method to address the high genetic heterogeneity of these disorders. It is likely that trio WES could potentially yield a greater diagnostic rate. Benefits for patients with a genetic diagnosis included ending the diagnostic odyssey and improved prognostic and reproductive counseling. 


\section{Oral 5}

\section{Kinesin-3 Family Member 1A (KIF1A)-Associated Neurological Disorders (KAND): Past, Present and Future}

Simranpreet Kaur ${ }^{1,2}$, Nicole J. Van Bergen ${ }^{1,2}$, Alejandro Hidalgo-Gonzalez ${ }^{3}$, Holly Voges ${ }^{3}$, Kristen J. Verhey ${ }^{4}$, Cameron J. Nowell ${ }^{5}$, Breane Budaitis ${ }^{6}$, Yang Yue ${ }^{4}$, Carolyn Ellaway ${ }^{7,8}$, Nicola Brunetti-Pierri ${ }^{9,10}$, Gerarda Cappuccio ${ }^{9,10}$, Irene Bruno ${ }^{11}$, Lia Boyle ${ }^{12}$, Vincenzo Nigro ${ }^{11}$, Annalaura Torella ${ }^{11}$, Tony Roscioli ${ }^{13,14}$, Mark J. Cowley ${ }^{15}$, Sean Massey ${ }^{1}$, Matthew D. Burton ${ }^{18}$, Bitten Schonewolf-Greulich ${ }^{19}$, Zeynep Tümer ${ }^{19}$, Zornitza Stark ${ }^{2,30}$, Natasha Brown ${ }^{30}$, Di Milnes ${ }^{20}$, Jason Pinner ${ }^{21}$, Rani Sachdev ${ }^{21}$ Michael C. Kruer ${ }^{22,23}$, Somayeh Bakhtiari ${ }^{22,23}$, Bertrand Isidor ${ }^{24}$, Frédéric Bilan ${ }^{25}$, Wendy K. Chung ${ }^{26}$, Wendy A. Gold ${ }^{27,28,29}$ and John Christodoulou ${ }^{1,2,7,30}$

${ }^{1}$ Brain and Mitochondrial Research Group, Murdoch Children's Research Institute, Royal Children's Hospital, Melbourne, Australia, ${ }^{2}$ Department of Paediatrics, University of Melbourne, Melbourne, Australia, ${ }^{3}$ Disease Modelling and Drug Discovery Facility, Murdoch Children's Research Institute, Royal Children's Hospital, Melbourne, Australia, ${ }^{4}$ Department of Cell and Developmental Biology, University of Michigan Medical School, Ann Arbor, USA, ${ }^{5}$ Drug Discover Biology, Monash Institute of Pharmaceutical Sciences, Monash University, Melbourne, Australia, ${ }^{6}$ Cellular and Molecular Biology Program, University of Michigan Medical School, Ann Arbor, USA, ${ }^{7}$ Discipline of Genomic Medicine, School of Medical Sciences, Faculty of Medicine and Health, University of Sydney, Camperdown, Australia, ${ }^{8}$ Western Sydney Genetics Program, Children's Hospital at Westmead, Sydney, Australia, ${ }^{9}$ Department of Translational Medicine, University of Naples 'Federico II', Naples, Italy, ${ }^{10}$ Telethon Institute of Genetics and Medicine, Pozzuoli (NA), Italy, ${ }^{11}$ Department of Precision Medicine, University of Campania 'Luigi Vanvitelli', Campania, Italy, ${ }^{12}$ Division of Molecular Genetics, Columbia University Irving Medical Center, New York, USA, ${ }^{13}$ New South Wales Health Pathology, Sydney, Australia, ${ }^{14}$ Neuroscience Research Australia, University of New South Wales, Sydney, Australia, ${ }^{15}$ Kinghorn Centre for Clinical Genomics, Garvan Institute of Medical Research, Sydney, Australia, ${ }^{16}$ St Vincent's Clinical School, University of New South Wales, Sydney, Australia, ${ }^{17}$ Children's Cancer Institute, Lowy Cancer Research Centre, University of New South Wales, Sydney, Australia, ${ }^{18}$ Flow Cytometry and Imaging facility, Murdoch Children's Research Institute, Royal Children's Hospital, Melbourne, Australia, ${ }^{19}$ Kennedy Center, Department of Clinical Genetics, Copenhagen University Hospital, Rigshospitalet, Denmark, ${ }^{20}$ Genetic Health Queensland, Royal Brisbane \& Women's Hospital, Brisbane, Australia, ${ }^{21}$ Centre for Clinical Genetics, Sydney Children's Hospital, Randwick, Australia, ${ }^{22}$ College of Medicine, University of Arizona, Phoenix, USA, ${ }^{23}$ Barrow Neurological Institute, Phoenix Children's Hospital, Phoenix, USA, ${ }^{24}$ L'institut du Thorax, INSERM, CNRS, UNIV Nantes, CHU de Nantes, France, ${ }^{25}$ Neurovascular unit and cognitive disorders, University of Poitiers, Poitiers, France, ${ }^{26}$ Departments of Paediatrics and Medicine, Columbia University Medical Center, New York, USA, ${ }^{27}$ Molecular Neurobiology Research Laboratory, Kids Research, Children's Hospital at Westmead, and The Children's Medical Research Institute, Sydney, Australia, ${ }^{28}$ Kids Neuroscience Centre, Kids Research, Children's Hospital at Westmead, Sydney, Australia, ${ }^{29}$ School of Medical Sciences and Discipline of Child and Adolescent Health, Faculty of Medicine and Health, The University of Sydney, Australia and ${ }^{30}$ Victorian Clinical Genetics Services, Royal Children's Hospital, Melbourne, Australia

Background: Defects in the motor domain of kinesin-3 family member 1A (KIF1A), a neuron-specific ATP-dependent anterograde axonal transporter of synaptic cargo, are well recognized to cause KIF1A-Associated Neurological Disorders (KAND). Due to resemblance with other neurological disorders, a significant number of children remain either misdiagnosed or undiagnosed. Moreover, there are currently no specific therapies for KAND. Aims: Our aims are to uncover pathogenic variants in KIF1A in individuals with complex neurological disorders, to assess functional consequences of identified variants on KIF1A function and to establish tools for high-throughput screening (HTS) of small molecules to improve KIF1A function. Methods: We used next generation sequencing techniques to screen individuals with KAND and related disorders. Functional validation of variants was carried out using a range of assays including the in vitro neurite tip accumulation and microtubule gliding assay. Results: Through various collaborations, we have identified 31 KAND individuals with 18 different variants, all clustered in the critical motor domain of the protein. All of these variants showed a similar functional phenotype, namely a significant reduction in the intracellular movement of the fluorescent tagged KIF1A motor domain when expressed in SH-SY5Y cells. We have now established a robust and efficient model compatible with a HTS approach to identify small molecules that could improve the function of KIF1A. Conclusions: This study has further expanded the phenotypic spectrum associated with pathogenic variants involving the KIF1A motor domain. The ultimate outcome of the project will be to identify compounds to improve the molecular function of defective KIF1A.

\section{Oral 6 \\ Expanded Preconception Carrier Screening - Results From a Wa Pilot Program}

Samantha Edwards ${ }^{1}$, Royston Ong ${ }^{1}$, Georgina Hollingsworth ${ }^{1}$, Karen Harrop ${ }^{2}$, Sarah Moore ${ }^{3}$, Benjamin Kamien ${ }^{2,4}$, Nicholas Pachter ${ }^{2,5}$, Mark Davis ${ }^{5}$, Karen Carpenter ${ }^{5}$, John Beilby ${ }^{5}$ and Nigel Laing ${ }^{1,5}$

${ }^{1}$ Harry Perkins Institute Of Medical Research, Perth, Australia, ${ }^{2}$ Genetic Services of Western Australia, King Edward Memorial Hospital, Department of Health WA, Perth, Australia, ${ }^{3}$ Rural Clinical School, University of Western Australia, Perth, Australia, ${ }^{4}$ UWA Medical School, University of Western Australian, Perth, Australia and ${ }^{5}$ Department of Diagnostic Genomics, PathWest Laboratory Medicine, WA Department of Health, Perth, Australia

Background: Expanded preconception carrier screening (EPCS) assesses the chance a couple will have a child affected with a recessive genetic condition regardless of family history. Next generation sequencing technologies make screening hundreds of conditions simultaneously affordable. This pilot study aimed to determine the requirements for successful implementation of EPCS in the public health system of Western Australia. Methods: 225 couples planning a pregnancy were screened for 425 severe life limiting, or chronic recessive conditions, with onset in infancy or early childhood. Couples were recruited from the Perth and Busselton regions through general practitioner, clinical genetic and private genetic counseling services. Couples received a 'high-risk' or 'low-risk' result. Individual carrier-status was not reported. Thirteen known high-risk couples were analyzed as positive controls, with the data analyzed blind to validate the laboratory processes. Outcomes: In the 225 couples sequenced, 520 pathogenic variants were identified: range 0-5 variants (average 1.2) per individual. 312 of the 450 individuals were carriers of at least one condition. Of 13 known high-risk couples, 8 were correctly identified. Five were incorrectly assessed as low risk, highlighting inadequate sharing of variant data in reference databases as a major issue. Analysis identified 7 new high-risk couples, a population frequency of 1 in 32 , which is at the higher end of previous publications. 17 couples reported pregnancy following sample collection and before testing was complete, emphasising the need for a reliable service with rapid result delivery. Health professional evaluation demonstrated increased knowledge and confidence in genetic counseling with adequate support. 


\section{Oral 7 \\ Unique Genotype-Dependent Trajectories in Retinal Lesion Area Based on Ultrawide-Field Autofluorescence Evaluation in Stargardt Disease (STGD1)}

Rachael Heath Jeffery ${ }^{1}$, Jennifer Thompson ${ }^{2}$, Terri McLaren², Tina Lamey ${ }^{2}$, John De Roach $^{2}$ and Fred Chen ${ }^{1,3,4}$

${ }^{1}$ Centre for Ophthalmology and Visual Science (incorporating Lions Eye Institute), The University of Western Australia, Perth, Australia, ${ }^{2}$ Australian Inherited Retinal Disease Registry and DNA Bank, Department of Medical Technology and Physics, Sir Charles Gairdner Hospital, Perth, Australia, ${ }^{3}$ Department of Ophthalmology, Royal Perth Hospital, Perth, Australia and ${ }^{4}$ Department of Ophthalmology, Perth Children's Hospital, Perth, Australia

Background: Stargardt disease (STGD1) occurs in 1:10,000 and is the most common cause of genetic blindness in working age groups. The area of retina affected by STGD1 ranges from involvement of the fovea to widespread chorioretinal atrophy. Ultrawide-field retinal autofluorescence (UWF-AF) imaging is widely accessible for evaluating the proportion of retina affected by the disease. Aim: To correlate the area of affected retina and age on UWF-AF imaging with allele severity in genetically confirmed STGD1. Methods: Patients with biallelic STGD1 variants were recruited. Ophthalmological assessment, UWF-AF imaging and genetic analysis using MVL panels (Oregon) were performed. The proportion of retina affected was calculated after marking of lesion border in UWF-AF images. Pathogenicity was assessed according to ACMG criteria and allele severities were classified based on current literature. Lesion size (\% of total retinal area) was plotted against patients' age for the most common alleles. Results: Seventy STGD1 patients (32 male; mean age 43 ) with a mean visual acuity of $6 / 48$ were included. Patients with biallelic null/severe variants $(n=21)$ demonstrated rapid increase in lesion size during the second decade to $>50 \%$ and continued to 65 $-75 \%$ by sixth decade. Those with c.6079C $>\mathrm{T} \quad(n=10)$ and c. [2588G $>$ C; $5603 \mathrm{~A}>\mathrm{T}] \quad(n=7)$ had lesion size reaching $35-55 \%$ by the third and sixth decades, respectively. Those with c.5882G $>\mathrm{A}(n=6)$ and c.5603A $>\mathrm{T}(n=11)$ had a relatively stable lesion size reaching $1 \%$ and $13 \%$ of the total retinal area respectively. Discussion/Conclusion: UWF-AF has a role in predicting allele severity as shown by unique trajectories of lesion size for common alleles.

\section{Oral 8}

Scaling National and International Improvement in Virtual Gene Panel Curation Using a Collaborative Approach to Discrepancy Resolution

Zornitza Stark ${ }^{1,2,3}$, Rebecca Foulger ${ }^{4,5}$, Eleanor Williams ${ }^{4,5}$, Bryony Thompson ${ }^{6}$, Chirag Patel $^{7}$, Ivone U. S. Leong ${ }^{4}$, Louise C. Daugherty ${ }^{4,5}$, Sarah Leigh ${ }^{4}$, Arina Puzriakova ${ }^{4}$, Catherine Snow ${ }^{4,5}$, Chris Boustred ${ }^{4}$, Olivia Niblock ${ }^{4}$, Antonio RuedaMartin ${ }^{4}$, Oleg Gerasimenko ${ }^{4}$, Kevin Savage ${ }^{4}$, William Bellamy ${ }^{4}$, Victor San Kho Lin ${ }^{8}$, Roman Valls ${ }^{8}$, Lavinia Gordon ${ }^{8}$, Helen Brittain ${ }^{4}$, Richard H. Scott ${ }^{4}$, Ellen R. A. Thomas ${ }^{4}$ Anna Lisa Taylor Tavares ${ }^{4}$, Zerin Hyder ${ }^{4}$, Meriel McEntagart ${ }^{4}$, Elena Savva ${ }^{1}$, Crystle Lee $^{1}$, Paul De Fazio ${ }^{1}$, Ain Roesley ${ }^{1}$, Ivan Macciocca ${ }^{1}$, Susan M. White ${ }^{1,2}$, Tiong Tan ${ }^{1,2}$, Alison Yeung ${ }^{1,2}$, Lilian Downie ${ }^{1,2,6}$, Sebastian Lunke ${ }^{1,2}$, Jane Deller ${ }^{9}$, Zandra C. Deans $^{9,10}$, Sue L. Hill' ${ }^{9}$, Mark J. Caulfield ${ }^{4}$, Kathryn N. North ${ }^{1,2,3}$, Augusto Rendon ${ }^{4,5}$, Oliver Hofmann ${ }^{8}$ and Ellen M. McDonagh ${ }^{4}$

${ }^{1}$ Victorian Clinical Genetics Services, Melbourne, Australia, ${ }^{2}$ The University of Melbourne, Melbourne, Australia, ${ }^{3}$ Australian Genomics Health Alliance, Melbourne, Australia, ${ }^{4}$ Genomics England, London, United Kingdom, ${ }^{5}$ Genomics England, Biodata Innovation Centre, Cambridge, United Kingdom, ${ }^{6}$ Royal Melbourne Hospital, Melbourne, Australia, ${ }^{7}$ Genetic Health Queensland, Brisbane, Australia, ${ }^{8}$ Centre for Cancer Research, University of Melbourne, Victorian Comprehensive Cancer Centre, Melbourne, Australia, ${ }^{9} \mathrm{National}$ Health Service England, London, United Kingdom and ${ }^{10} \mathrm{National}$ Health Service Improvement, London, United Kingdom
Robust gene-disease validity assessments are the foundation of accurate variant interpretation in diagnostic genomics. Virtual gene panels for specific clinical indications improve the efficiency of genomic analysis, yet there is wide variability in the processes for panel design and maintenance, and in panel content. Harmonization efforts are hampered by siloed knowledge repositories, and lack of agreed terminology and standards. Aim: To harmonize virtual panel content across multiple diagnostic genomic services and identify areas for practice improvement. Methods: We undertook a systematic comparison of gene panel content across Genomics England PanelApp and PanelApp Australia, open platforms that capture structured gene-disease validity assessments and use the same evidence thresholds for gene inclusion in panels. The Genomics England PanelApp contains 325 panels developed for the 100,000 Genomes Project and the NHS Genomic Medicine Service, whereas PanelApp Australia contains 249 panels developed by Australian Genomics projects and diagnostic laboratories/clinical services. Results: Comparing 80 panel pairs covering comparable clinical indications identified 2016 discordant gene inclusions and ratings. Reassessment resulted in exchange of reviews to support inclusion of 921 genes in diagnostic analysis, and 1095 assessments identifying only limited evidence for diagnostic use. Common reasons for discrepancies included differences in panel scope definition, differential weighting of functional evidence, and challenges in systematic identification of new evidence establishing or refuting gene-disease associations. Conclusions: We demonstrate the utility of a scalable approach to improving gene-disease validity assessments and virtual gene panel content that highlights the benefits of open platforms for harmonized data capture and shared expert curation.

\section{Oral 9}

\section{Lower Penetrance Estimates for Neurosusceptibility Loci - an Improved Formula}

Shuxiang Goh ${ }^{1}$, Rhys Bowden ${ }^{2}$ and Edwin Kirk $^{3}$

${ }^{1}$ Liverpool Hospital, Sydney, Australia, ${ }^{2}$ Sydney Children's Hospital, Sydney, Australia and ${ }^{3}$ Monash University, Melbourne, Australia

Penetrance has been defined as the proportion of individuals with a given genetic change who display a phenotypic change. We show that this is an ambiguous definition that leads to two different interpretations. One interpretation is used by clinicians and counselors (essentially 'the likelihood that the variant will cause a phenotype'). A different mathematical interpretation has been used to calculate penetrance based on Bayes' theorem (essentially 'the likelihood the variant may been seen in association with a phenotype'). The latter definition encompasses individuals who have the variant by chance - associated with but not causal of the phenotype. The interpretations are incompatible. As a result, many of the published penetrance estimates for neurosusceptibility loci, intellectual disability, schizophrenia and autism are overestimated. Methods: We provide a mathematical rationale for a more clinically meaningful formula for penetrance. In addition to creating a more accurate formula, we also improve the data used in the formula. We justify our approach in changing the background rate of any physical or intellectual disability from $5.12 \%$ used by some authors, to $1.1 \%$ for intellectual disability. This results in penetrance estimations for neurosusceptibility loci that are approximately 5-fold lower in some instances. Results: When the two approaches are combined, we find that the penetrance for most neurosusceptibility loci are markedly decreased. Some previously low-penetrant neurosusceptibility loci are recalculated as having a penetrance of $0 \%$. Conclusion: Most 
neurosusceptibility loci have a lower penetrance than previously estimated. This has diagnostic and counseling implications in both the prenatal and postnatal settings.

\section{Oral 10 \\ The Patient With 41 Reports: Analysis of Laboratory Exome Sequencing Reporting of A 'Virtual Patient'}

Danya F. Vears ${ }^{1,2,3,4}$, Martin Elferink ${ }^{5}$, Marjolein Kriek ${ }^{6}$, Pascal Borry ${ }^{3,4}$ and Koen L. van Gassen $^{5}$

${ }^{1}$ Biomedical Ethics Research Group, Murdoch Children's Research Institute, Melbourne, Australia, ${ }^{2}$ Melbourne Law School, University of Melbourne, Melbourne, Australia, ${ }^{3}$ Center for Biomedical Ethics and Law, Department of Public Health and Primary Care, KU Leuven, Leuven, Belgium, ${ }^{4}$ Leuven Institute for Human Genetics and Society, Leuven, Belgium, ${ }^{5}$ Department of Genetics, University Medical Center Utrecht, Utrecht, the Netherlands and ${ }^{6}$ Department of Clinical Genetics, Leiden University Medical Center, Leiden, the Netherlands

Although recommendations for both variant classification and the components that should be included in genomic sequencing reports issued by laboratories exist, there has been no systematic exploration of their use in practice. To investigate the degree of variation in reporting, we created a 'virtual patient-parent trio' by in silico merging eight disease-causing variants from patients into 'normal' background exomes. Two variants related to the patient's primary phenotype: HDAC8 and BICD2. We sent sequence data and the patient's phenotypic details (developmental delay, dysmorphic features, cardiac hypertrophy), to laboratories worldwide and asked them to issue a diagnostic report. Reports were coded for the presence of reporting components using deductive content analysis. Of the 41 laboratories representing 17 countries that issued reports, $90 \%$ mentioned the transcript reference number and $78.6 \%$ used HGVS nomenclature. Reporting of quality control statistics and technical information was poor $(46.3 \%)$. Although $75.6 \%$ clearly stated the classification of all reported variants, few reports listed extensive evidence supporting the variant classification. Only $53.1 \%$ of the laboratories that reported unsolicited or secondary findings gave advice regarding health-related follow up and $20.5 \%$ gave advice regarding cascade testing for relatives. While $51.9 \%$ of the 27 laboratories that reported the BICD2 variant correctly classified it as likely pathogenic, $7.4 \%$ classified it as pathogenic and $25.9 \%$ as variant of uncertain significance. We identified considerable variability in both the components laboratories included in their reports and also their classification of variants. This suggests that existing guidelines are not being used correctly with significant implications for patient care.

\section{Oral 11 \\ Functional Studies Coupled with Detailed Clinical Phenotyping Provides New Insights Into CLCN4- Encephalopathy in a Cohort of 29 Individuals}

Elizabeth Palmer ${ }^{1,2}$, Michael Pusch $^{3}$, Alessandra Picollo ${ }^{3}$, Vanessa Suckow ${ }^{4}$, Andre Megarbane ${ }^{5}$, Mathilde Nizon ${ }^{6}$, Benjamin Cogne $^{6}$, Fowzan Alkuraya ${ }^{7}$, Aziza Chedrawi ${ }^{7}$, Hannah Stamberger ${ }^{8}$, Sarah Weckhuysen ${ }^{8}$, Berten Ceulemans ${ }^{8}$, S. Rajagopalan ${ }^{9}$, Stephanie Arpin ${ }^{10}$, Martine Raynaud ${ }^{10}$, Constance S. Motter ${ }^{11}$, Catherine Ward-Melver ${ }^{11}$, Katrien Janssens ${ }^{8}$, Marije Meuwissen ${ }^{8}$, Diane Beysen ${ }^{8}$, Marjolein Willemsen ${ }^{12}$, Nicola Dikow ${ }^{13}$, Mona Grimmel ${ }^{14}$, Tobias B. Haack ${ }^{14}$, Emma Clement ${ }^{15}$, Amy McTague ${ }^{15}$, David Hunt ${ }^{16}$, Sharron Townshend ${ }^{17}$, Michelle Ward ${ }^{17}$, Linda Richards ${ }^{18}$, Cas Simonds ${ }^{19}$, Gregory Costain ${ }^{20}$, Lucie Dupuis ${ }^{20}$, Roberto Mendoza-Londono ${ }^{20}$, Tracy Dudding-Byth ${ }^{21}$, Jackie Boyle ${ }^{21}$, Ruth Armstrong ${ }^{22}$, Carol Saunders ${ }^{31}$, Salima El Chehadeh ${ }^{23}$, Bénédicte Gerard ${ }^{24}$, Rani Sachdev ${ }^{1}$,
Rebecca Macintosh ${ }^{1}$, Melissa Carter ${ }^{25}$, Luis Rohena ${ }^{26}$, Charlotte Ockeloen ${ }^{12}$, S. D. Kroft ${ }^{27}$, Mike Field ${ }^{21}$, Jill Rosenfeld ${ }^{28}$, Christian Beetz ${ }^{30}$, Caitlin Forwood ${ }^{1}$, Matthew Nguyen ${ }^{2}$, Rolph Pfundt ${ }^{12}$, Kirsty McWalter ${ }^{29}$ and Vera Kalscheuer ${ }^{4}$

${ }^{1}$ Sydney Children's Hospital Network, Sydney, Australia, ${ }^{2}$ School of Women's and Children's Health, University of New South Wales, Sydney, Australia, ${ }^{3}$ Istituto di Biofisica CNR, Genova, Italy, ${ }^{4}$ Max Planck Institute for Molecular Genetics, Berlin, Germany, ${ }^{5}$ Department of Human Genetics, Gilbert and RoseMarie Ghagoury School of Medicine, Lebanese American University, Byblos, Lebanon, ${ }^{6} \mathrm{CHU}$ Nantes, Service de Génétique Médicale, Nantes, France, ${ }^{7}$ King Faisal Specialist Hospital and Research Center, Riyadh, Saudi Arabia,

${ }^{8}$ Antwerp University Hospital, Antwerp, Belgium, ${ }^{9}$ Liverpool Hospital, Sydney, Australia, ${ }^{10}$ Brain, Université de Tours, Tours, France, ${ }^{11}$ Akron Children's Hospital, Akron, USA, ${ }^{12}$ Radboud University Medical Center, Nijmegen, the Netherlands, ${ }^{13}$ Heidelberg University, Heidelberg, Germany, ${ }^{14}$ Institute of Medical Genetics and Applied Genomics, Tuebingen, Germany, ${ }^{15}$ Great Ormond Street Hospital for Children, London, UK, ${ }^{16}$ Princess Anne Hospital, Southampton, UK, ${ }^{17}$ King Edward Memorial Hospital, Perth, Australia, ${ }^{18}$ Queensland Brain Institute, The University of Queensland, Brisbane, Australia, ${ }^{19}$ Translational Bioinformatics group, Murdoch Children's Research Institute, Melbourne, Australia, ${ }^{20}$ Division of Clinical and Metabolic Genetics, The Hospital for Sick Children, Toronto, Canada, ${ }^{21}$ Genetics of Learning Disability Service, Newcastle, Australia, ${ }^{22}$ East Anglian Medical Genetics Service, Clinical Genetics, Addenbrooke's Treatment Centre, Addenbrooke's Hospital, Cambridge, UK, ${ }^{23}$ Service de Génétique Médicale Hôpital de Hautepierre, Strasbourg, France, ${ }^{24}$ Institut de Génétique Médicale d'Alsace (IGMA) Laboratoires de Diagnostic Génétique, Unité de génétique moléculaire Nouvel Hôpital Civil, Strasbourg, France, ${ }^{25}$ University of Ottawa, Ottawa, Canada, ${ }^{26}$ University of Texas Health Science Center at San Antonio, San Antonio, USA, ${ }^{27}$ Pluryn, Groesbeek, Netherlands, ${ }^{28}$ Baylor College of Medicine, Houston, USA, ${ }^{29}$ GeneDx, Gaithersburg, USA, ${ }^{30}$ CENTOGENE, Rostock, Germany and ${ }^{31}$ Children's Mercy Hospital and Clinics Kansas City, Kansas City, USA

Missense and loss-of-function variants in the X-chromosome-linked CLCN4 gene, resulting in reduced function of the encoded chloride/ proton ion-exchanger CIC-4, was recently demonstrated by our group to cause intellectual disability, seizures and behavior disorders in males and females. Subsequently we received multiple clinical queries regarding the pathogenicity of novel missense variants, often with pressing reproductive implications. Through international clinical matchmaking and interrogation of publicly available variant databases we assembled a database of 42 rare (absent from gnomAD), previously unreported CLCN4 missense variants of uncertain significance, and modelled the predicted effect on protein structure and electrophysiological properties of mutated CIC-4 in a Xenopus oocyte model. 55\% (23/42) of the variants resulted in a reduction of current size or a shift of voltage dependency. For 14 of these we were able to obtain detailed clinical information and found consistent segregation data/neuropsychiatric phenotype. $45 \%(19 / 42)$ did not result in a loss of ion-exchanger function, and yet for several of these variants a strong clinical suspicion of pathogenicity remained, due to compatible neuropsychiatric phenotype with concordant segregation, de novo status and/or recurrence in unrelated families. Modification of the experimental conditions demonstrated that 6 of these variants resulted in a toxic gain-of-function: a critical new pathophysiological insight guiding accurate pathogenicity assessment and advanced therapeutic development. We present detailed clinical data on, in total, 29 individuals (missense and loss-of-function variants), and broaden the phenotypic spectrum, including a de novo recurrent variant in females causing extreme growth restriction: a finding which has guided clinical management of 4 girls internationally. 


\section{Oral 12 \\ The Psychosocial Impact of Receiving Unexpected Genetic Findings From Research: Experiences Of Lifepool Participants}

Mary-Anne Young ${ }^{1}$, Rowan Forbes Shepherd ${ }^{3}$, Angela Pearce ${ }^{1}$, Erin Tutty ${ }^{4}$, Ian Campbell ${ }^{5}$, Lisa Devereux ${ }^{5}$, Paul James ${ }^{4}$ and Laura Forrest ${ }^{4}$

${ }^{1}$ Kinghorn Centre for Clinical Genomics, Garvan Institute of Medical Research, Sydney, Australia, ${ }^{2}$ University of New South Wales, Sydney, Australia, ${ }^{3}$ Sir Peter MacCallum Department of Oncology, University of Melbourne, Melbourne, Australia, ${ }^{4}$ Parkville Familial Cancer Centre, Peter MacCallum Cancer Centre and the Royal Melbourne Hospital, Melbourne, Australia and ${ }^{5}$ Sir Peter MacCallum Department of Oncology, University of Melbourne, Melbourne, Australia

Background: The LifePool research program gathers data from women undertaking population-level breast cancer screening from BreastScreen in Victoria, Australia. One aim is to determine the population prevalence of germline variants in 11 Hereditary Breast and Ovarian Cancer (HBOC) genes. Women who consent to be contacted about clinically actionable genetic information are informed via an evidence-based two-tier notification process. For these women, learning that they carry a pathogenic germline variant is likely to be unexpected and possibly distressing. However, there is limited research on how women experience, value, accept and adapt to this information. Aim: To explore how women at population-risk of breast cancer experience receiving clinically actionable genetic information identified in a research setting. Methods: A mixed-methods design was used; here we report the qualitative findings. Semistructured interviews were conducted with women who were notified of their result by lifepool. Team-based reflexive thematic analysis was used to analyze interview data. Results: Thirty-one women were interviewed (Mage 62.5 years) on average 2.3 years (range 0.3-5.1) after receiving genetic information from lifepool. Findings indicate this information was often unexpected, although not experienced as traumatic; the genetic information was viewed as valuable as it empowered women to make informed decisions about their personal and familial health. Adaptation was influenced by the availability of risk management strategies, well-received family communication and availability of the telephone genetic counseling service. Conclusion: Findings suggest being notified of clinically actionable genetic information for HBOC via a population-based genetic testing program is acceptable and the information is empowering.

\section{Oral 13 \\ Pathogenic Variants in MRPL39 as A Novel Cause of Mitochondrial Disease}

S. S. C. Amarasekera ${ }^{1,2}$, D. H. Hock ${ }^{3}$, N. J. Lake ${ }^{1,4}$, S. E. Calvo ${ }^{5,6,7}$, E. I. Krzesinski ${ }^{8,9}$, D. J. Amor ${ }^{1,2}$, M. C. Fahey ${ }^{8,9}$, C. Simons ${ }^{1,10}$, V. K. Mootha ${ }^{5,6,7}$, M. Lek ${ }^{4}$, S. Lunke ${ }^{11,12}$, Z. Stark ${ }^{2,11,12}$, D. A. Stroud ${ }^{3}$, J. Christodoulou ${ }^{1,2,11,12}$, D. R. Thorburn ${ }^{1,2,11,12}$ and A. G. Compton ${ }^{1,2}$

${ }^{1}$ Murdoch Children's Research Institute, Royal Children's Hospital, Melbourne, Australia, ${ }^{2}$ Department of Paediatrics, University of Melbourne, Melbourne, Australia, ${ }^{3}$ Department of Biochemistry and Molecular Biology, Bio21 Molecular Science and Biotechnology Institute, University of Melbourne, Melbourne, Australia, ${ }^{4}$ Department of Genetics, Yale School of Medicine, New Haven, USA, ${ }^{5}$ Broad Institute, Cambridge, USA, ${ }^{6}$ Howard Hughes Medical Institute and Department of Molecular Biology, Massachusetts General Hospital, Boston, USA, ${ }^{7}$ Department of Systems Biology, Harvard Medical
School, Boston, USA, ${ }^{8}$ Monash Genetics, Monash Health, Melbourne, Australia, ${ }^{9}$ Department of Paediatrics, Monash University, Melbourne, Australia, ${ }^{10}$ Institute for Molecular Bioscience, The University of Queensland, Brisbane, Australia, ${ }^{11}$ Victorian Clinical Genetics Services, Murdoch Children's Research Institute, Melbourne, Australia and ${ }^{12}$ Australian Genomics Health Alliance, Royal Children's Hospital, Melbourne, Australia

Background: Mitochondrial ribosomes (mitoribosomes) synthesize 13 mitochondrial DNA encoded proteins essential for cellular energy generation via oxidative phosphorylation (OXPHOS). Mitoribosomes are composed of $\sim 80$ proteins, with biallelic mutations in 11 of these thus far demonstrated to cause severe recessive multisystemic mitochondrial disorders. We have identified MRPL39, encoding a protein component of the mitoribosomal large subunit, as a novel disease gene associated with Leigh and Leigh-like syndrome. Methods and Results: Two unrelated patients with Leigh or Leigh-like syndrome each had single rare heterozygous variants (c.912+5G $>$ A and c.526delT) identified by exome sequencing in MRPL39, predicted to result in premature stop codons and nonsense mediated decay (NMD). Subsequent whole genome sequencing (WGS) uncovered a rare heterozygous deep intronic variant (c.589-924G>A) present in both patients, which created a cryptic exon and also underwent NMD. The variants were confirmed to be biallelic by segregation analysis. RNA sequencing (RNAseq) and full length cDNA analysis of patient fibroblasts established aberrant splicing and aberrant expression corresponding to each of the mutations identified. Steady state levels of large mitoribosome subunit proteins (including MRPL39) and OXPHOS complexes were shown to be decreased in patient fibroblasts by quantitative proteomics and western blotting. In contrast, small mitoribosome subunit proteins were unchanged. Conclusion: This study identified biallelic loss-of-function mutations in MRPL39 as a novel cause of mitochondrial disease via mitoribosomal large subunit instability. It highlights the utility of WGS, RNAseq and quantitative proteomics for gene prioritization plus discovery and validation of pathogenic variants beyond the exome.

\section{Oral 14}

\section{Germline and Mosaic Variants in PRKACA and PRKACB Cause A Multiple Congenital Malformation Syndrome}

Adrian Palencia-Campos ${ }^{1,2,26}$, Phillip Aoto ${ }^{3,26}$, Erik Machal ${ }^{4,26}$, Rivera-Barahona ${ }^{1,2}$, Patricia Soto-Bielicka ${ }^{1}$, Daniela Bertinetti ${ }^{4}$, Blaine Baker ${ }^{3}$, Lily $\mathrm{Vu}^{3}$, Francesca PiceciSparascio ${ }^{5}$, Isabella Torrente ${ }^{5}$, Eveline Boudin ${ }^{6}$, Silke Peeters ${ }^{6}$, Wim Van Hul ${ }^{6}$ Celine Huber ${ }^{7,8}$, Dominique Bonneau ${ }^{9,10}$, Michael Hildebrand ${ }^{11,12}$, Matthew Coleman ${ }^{11}$, Melanie Bahlo ${ }^{13,14}$, Mark Bennett ${ }^{11,13,14}$, Amy Schneider ${ }^{11}$, Ingrid Scheffer ${ }^{11,12,15}$, Maria Kibaek ${ }^{16}$, Britta Kristiansen ${ }^{17}$, Mahmoud Issa ${ }^{18}$, Mental Mehrez ${ }^{19}$, Samira Ismail ${ }^{18}$, Jair Tenorio ${ }^{2,20,21}$, Gaoyang Li ${ }^{22}$, Bjorn Skalhegg ${ }^{22}$, Ghada Otaify ${ }^{18}$, Samia Temtamy ${ }^{18}$, Mona Aglan ${ }^{18}$, Jonch ${ }^{17}$, Alessandro De Luca ${ }^{5}$, Geert Mortier ${ }^{6,23}$, Valerie Cormier-Daire ${ }^{7,8}$, Alban Ziegler ${ }^{9,10}$, Mathew Wallis ${ }^{24,25}$, Pablo Lupunzina ${ }^{2,20,21}$, Friedrich Herberg ${ }^{4}$, Susan Taylor ${ }^{3,26}$ and Victor Ruiz-Perez ${ }^{1,2,20,21}$

${ }^{1}$ Instituto de Investigaciones Biomedicas "Alberto Sols," Consejo Superior de Investigaciones Cientificas (CSIC)—Universidad Autonoma de Madrid (UAM), Madrid, Spain, ${ }^{2}$ CIBER de Enfermedades Raras (CIBERER), Instituto de Salud Carlos III (ISCIII), Madrid, Spain, ${ }^{3}$ Department of Pharmacology, University of California, San Diego, San Diego, USA, ${ }^{4}$ Institute for Biology, Department of Biochemistry, University of Kassel, Kassel, Germany, ${ }^{5}$ Medical Genetics Unit, Casa Sollievo della Sofferenza Foundation, IRCCS, San Giovanni Rotondo, Italy, ${ }^{6}$ Department of Medical Genetics, University of Antwerp, Edegem, Belgium, ${ }^{7}$ Clinical Genetics and Reference Center for Skeletal Dysplasia, APHP, Necker-Enfants Malades Hospital, Paris, France, ${ }^{8}$ Universite De Paris, INSERM UMR1163, Institut Imagine, Paris, France, ${ }^{9}$ Biochemistry and Genetics Department, Angers Hospital, Angers, France, ${ }^{10}$ UCNRS 6015-INSERM U1083, 
MitoVasc Institute, Angers University, Angers, France, ${ }^{11}$ Epilepsy Research Centre, Department of Medicine, Austin Health, University of Melbourne, Melbourne, Australia, ${ }^{12}$ Murdoch Children's Research Institute, Melbourne, Australia, ${ }^{13}$ Population Health and Immunity Division, The Walter and Eliza Hall Institute of Medical Research, Melbourne, Australia, ${ }^{14}$ Department of Medical Biology, University of Melbourne, Melbourne, Australia, ${ }^{15}$ Department of Paediatrics, University of Melbourne, Royal Children's Hospital, and Florey Institute of Neuroscience and Mental Health, Melbourne, Australia, ${ }^{16}$ Children's Hospital of H.C. Andersen, Odense University Hospital, Odense, Denmark, ${ }^{17}$ Department of Clinical Genetics, Odense University Hospital, Odense, Denmark, ${ }^{18}$ Department of Clinical Genetics, Division of Human Genetics and Genome Research, Center of Excellence for Human Genetics, National Research Centre, Cairo, Eqypt, ${ }^{19}$ Department of Oro-dental Genetics, Division of Human Genetics and Genome Research. Center of Excellence for Human Genetics, National Research Centre, Cairo, Egypt, ${ }^{20}$ Instituto de Genetica Medicay Molecular (INGEMM)-IdiPAZ, Hospital Universitario La Paz, Madrid, Spain, ${ }^{21}$ European Reference Network on Rare Congenital Malformations and Rare Intellectual Disability, Ithaca, Luxembourg, ${ }^{22}$ Division for Molecular Nutrition, Institute for Basic Medical Sciences, University of Oslo, Oslo, Norway, ${ }^{23}$ Antwerp University Hospital, Edegem, Belgium, ${ }^{24}$ School of Medicine and Menzies Institute for Medical Research, University of Tasmania, Hobart, Australia, ${ }^{25}$ Clinical Genetics Service, Austin Health, Melbourne, Australia and ${ }^{26}$ Department of Chemistry and Biochemistry, University of California, San Diego, USA

PRKACA and PRKACB code for two catalytic subunits ( $\mathrm{Ca}$ and $\mathrm{Cb}$ ) of cAMP-dependent protein kinase A (PKA), a pleiotropic holoenzyme that regulates numerous fundamental biological processes such as metabolism, development, memory, and immune response. We report seven unrelated individuals presenting with a multiple congenital malformation syndrome in whom we identified heterozygous germline or mosaic missense variants in PRKACA or PRKACB. Three affected individuals were found with the same $P R K A C A$ variant, and the other four had different $P R K A C B$ mutations. In most cases, the mutations arose de novo, and two individuals had offspring with the same condition. Nearly all affected individuals and their affected offspring shared an atrioventricular septal defect or a common atrium along with postaxial polydactyly. Additional features included skeletal abnormalities and ectodermal defects of variable severity in five individuals, cognitive deficit in two individuals, and various unusual tumors in one individual. We investigated the structural and functional consequences of the variants identified in PRKACA and PRKACB through the use of several computational and experimental approaches, and we found that they lead to PKA holoenzymes which are more sensitive to activation by cAMP than are the wild-type proteins. Furthermore, expression of PRKACA or PRKACB variants detected in the affected individuals inhibited hedgehog signaling, thereby providing an underlying mechanism for the developmental defects observed in these cases. Our findings highlight the importance of both $\mathrm{Ca}$ and $\mathrm{Cb}$ subunits of PKA during human development.

\section{Oral 15 \\ The Australian Genomics Health Alliance Mitochondrial Flagship: Searching for what Lies Beneath}

John Christodoulou ${ }^{1,2}$, Naomi Baker ${ }^{2,3}$, Shanti Balasubramaniam ${ }^{4}$, Kaustuv Bhattacharya ${ }^{4}$, Drago Bratkovic ${ }^{5}$, David Coman ${ }^{6}$, Alison Compton ${ }^{1,2}$, Martin Delatycki ${ }^{2,3}$, Carolyn Ellaway ${ }^{4}$, Michael Fahey ${ }^{7}$, Janice Fletcher ${ }^{8}$, Ann Frazier $^{1,2}$, Roula Ghaoui ${ }^{8}$, Daniella Hock ${ }^{2,9}$, Maina Kava ${ }^{10}$, Nicole Lake ${ }^{1,11}$, Phillipa Lamont ${ }^{10,12}$, Joy Lee ${ }^{2,13}$, Professor Jim McGill ${ }^{6}$, Julie Panetta ${ }^{7}$, Liza Phillips ${ }^{8}$, Rocio Rius ${ }^{1,2}$, Michael Ryan ${ }^{14}$, Nicholas Smith ${ }^{5}$, David Stroud ${ }^{2,9}$, Michel Tchan ${ }^{15}$, Matthew Wallis ${ }^{16}$, Anne Marie Welch ${ }^{1}$, Christine Wools ${ }^{7}$ and David Thorburn ${ }^{1,2}$

${ }^{1}$ Murdoch Children's Research Institute, Melbourne, Australia, ${ }^{2}$ University of Melbourne, Melbourne, Australia, ${ }^{3}$ Victorian Clinical Genetics Service,
Melbourne, Australia, ${ }^{4}$ Sydney Children's Hospitals Network, Sydney, Australia, ${ }^{5}$ Women's and Children's Hospital, Adelaide, Australia, ${ }^{6}$ Queensland Children's Hospital and Wesley Hospital, Brisbane, Australia, ${ }^{7}$ Royal Melbourne Hospital, Melbourne, Australia, ${ }^{8}$ Royal Adelaide Hospital, Adelaide, Australia, ${ }^{9} \mathrm{Bio} 21$ Molecular Science and Biotechnology Institute, Melbourne, Australia, ${ }^{10}$ Perth Children's Hospital, Perth, Australia, ${ }^{11}$ Yale School of Medicine, New Haven, USA, ${ }^{12}$ Royal Perth Hospital, Perth, Australia, ${ }^{13}$ Royal Children's Hospital, Melbourne, Australia, ${ }^{14}$ Monash Biomedicine Discovery Institute, Monash University, Clayton, Australia, ${ }^{15}$ Westmead Hospital, Sydney, Australia and ${ }^{16}$ Tasmanian Clinical Genetics Service, Hobart, Australia

Background: With the advent of genomic technologies, the diagnostic landscape for mitochondrial respiratory chain disorders (RCD), notorious for their complexity, has changed dramatically. The Australian Genomics Health Alliance aims to bring genomic technologies to the clinic, and has established a Mitochondrial Disease Flagship. This focused national network has brought together clinicians, diagnostic and research scientists with specific interest and expertise in RCD to examine the clinical utility of genomic sequencing for the efficient diagnosis of RCD. Research Question: The challenge is how to tackle the cases that remain without a diagnosis, some of which have variants of uncertain significance in known disease genes (VUS), and others which have variants in genes of uncertain significance (GUS). Methods: We are using 'multi-omic' approaches to solve these difficult cases, including enzyme analyses, western blotting, quantitative proteomics, Blue-Native PAGE and RNA analyses. Results: Of the 135 pediatric and adult individuals recruited, based on modified Nijmegen criteria, we currently have a diagnostic yield of $\sim 37 \%$ in known disease genes, mostly known RCD genes, but around a quarter in non-RCD known disease genes. Examples of solved cases will be described, including individuals with novel variants in known genes such as MT-ATP6, MRPS34, PNPT1, ACAD9, COX15 and the ATAD3 gene locus. In addition, we are studying several novel candidate disease genes. Conclusions: Through the Mitochondrial Disease Flagship, we have demonstrated the power of a national networked approach, including functional approaches, to maximize the diagnostic yield for this complex group of disorders.

\section{Oral 16 \\ Bi-Allelic Mutations in NADSYN1 Cause Multiple Organ Defects and Expand the Genotypic Spectrum of Congenital NAD Deficiency Disorders}

Justin Szot ${ }^{1}$, Carla Campagnolo ${ }^{2}$, Ye $\mathrm{CaO}^{3,4,5,6}$, Kavitha Iyer ${ }^{1}$, Hartmut Cuny ${ }^{1,7}$, Thomas Drysdale ${ }^{8,9,10}$, Josue Flores-Daboub ${ }^{11}$, Weimin $\mathrm{Bi}^{3,6}$, Lauren Westerfield ${ }^{3}$, Pengfei Liu ${ }^{3,6}$, Tse Leung ${ }^{5}$, Kwong Choy ${ }^{4,12}$, Gavin Chapman ${ }^{1,7}$, Rui Xiao ${ }^{3,6}$, Victoria Siu ${ }^{2,8}$ and Sally Dunwoodie ${ }^{1,7,13}$

${ }^{1}$ Victor Chang Cardiac Research Institute, Sydney, Australia, ${ }^{2}$ Division of Medical Genetics, Department of Pediatrics, University of Western Ontario, London, Canada, ${ }^{3}$ Department of Molecular and Human Genetics, Baylor College of Medicine, Houston, USA, ${ }^{4}$ Department of Obstetrics and Gynaecology, The Chinese University of Hong Kong, Hong Kong, China, ${ }^{5}$ Obstetrics \& Gynaecology Centre, Hong Kong Sanatorium \& Hospital, Hong Kong, China, ${ }^{6}$ Baylor Genetics, Houston, USA, ${ }^{7}$ Faculty of Medicine, University of New South Wales, Sydney, Australia, ${ }^{8}$ Children's Health Research Institute, London, Canada, ${ }^{9}$ Department of Physiology and Pharmacology, Western University, London, Canada, ${ }^{10}$ Department of Paediatrics, Western University, London, Canada, ${ }^{11}$ Division of Medical Genetics, Department of Pediatrics, University of Utah, Salt Lake City, USA, ${ }^{12}$ The Chinese University of Hong Kong-Baylor College of Medicine Joint Center For Medical Genetics, Hong Kong, China and ${ }^{13}$ Faculty of Science, University of New South Wales, Sydney, Australia

Birth defects occur in up to $3 \%$ of all live births and are the leading cause of infant death. Here we present five individuals from four 
unrelated families, individuals who share similar phenotypes with disease-causal bi-allelic variants in NADSYN1, encoding NAD synthetase 1, the final enzyme of the nicotinamide adenine dinucleotide (NAD) de novo synthesis pathway. Defects range from the isolated absence of both kidneys to multiple malformations of the vertebrae, heart, limbs, and kidney, and no affected individual survived for more than three months postnatally. NAD is an essential coenzyme for numerous cellular processes. Bi-allelic loss-of-function mutations in genes required for the de novo synthesis of NAD were previously identified in individuals with multiple congenital abnormalities affecting the heart, kidney, vertebrae, and limbs. Functional assessments of NADSYN1 missense variants, through a combination of yeast complementation and enzymatic assays, show impaired enzymatic activity and severely reduced NAD levels. Thus, NADSYN1 represents an additional gene required for NAD synthesis during embryogenesis, and NADSYN1 has bi-allelic missense variants that cause NAD deficiency-dependent malformations. Our findings expand the genotypic spectrum of congenital NAD deficiency disorders and further implicate mutation of additional genes involved in de novo NAD synthesis as potential causes of complex birth defects.

\section{e-Poster Presentations}

\section{BIOCHEMICAL GENETICS}

\section{Importance of Functional Analysis in Reclassification of Compound Heterozygous VOUS in an Intermediate MSUD, Facilitating Prenatal Testing and Family Planning}

Bea Gutierrez ${ }^{1}$, Beena Devanapalli ${ }^{1}$, Mona Sajeev ${ }^{2}$, Sharon $\mathrm{Chin}^{3}$, Gladys $\mathrm{Ho}^{4}$, Bruce Bennetts ${ }^{4}$, Veronica Wiley ${ }^{5}$, Maria Fuller ${ }^{3}$, Adviye Ayper Tolun ${ }^{1}$ and Shanti Balasubramaniam²

${ }^{1}$ NSW Biochemical Genetics Service, Western Sydney Genetics Program, The Children's Hospital at Westmead, Sydney, Australia, ${ }^{2}$ Genetic Metabolic Disorders Service, Sydney Children's Hospital Network, Sydney, Australia, ${ }^{3}$ Genetics and Molecular Pathology, SA Pathology at Women's and Children's Hospital, Adelaide, Australia, ${ }^{4}$ Department of Molecular Genetics, Western Sydney Genetics Program, The Children's Hospital at Westmead, Sydney, Australia and ${ }^{5}$ NSW Newborn Screening Programme, Western Sydney Genetics Program, The Children's Hospital at Westmead, Sydney, Australia

Maple Syrup Urine Disease (MSUD) is a disorder affecting branched-chain amino acids. It presents in classical, intermittent and intermediate forms. Intermediate MSUD may be difficult to identify by newborn screening due to partial branched-chain alpha-ketoacid dehydrogenase deficiency. Case report: A female infant of non-consanguineous Caucasian parents presented with mild gross motor delay and poor head control aged 4 months, followed by seizures and hypoglycemia at 5 months. Plasma amino acid and urine organic acid analyses were consistent with a diagnosis of MSUD. Initial newborn screening returned elevated total leucine; however, second-tier testing reported minimal alloisoleucine, inconsistent with MSUD. Brain MRI at diagnosis showed diffuse hyperintense changes involving the medulla, pons, midbrain, and basal ganglia. EEG was mildly abnormal, with intermittent slowing noted bilaterally. Repeat MRI brain at 10 months after dietary intervention showed resolution of findings. Molecular analysis of BCKDHA, BCKDHB and DBT identified two VOUS (c.434-15_434-4del) and
c.365A $>$ G (p.Tyr122Cys) in DBT. The rate of leucine decarboxylation in fibroblasts was reduced, but not to the extent observed in affected MSUD patients, supporting an intermediate form of MSUD. Studies on mRNA splicing supported a deleterious effect of the c.434-15_434-4del variant. As this variant was shown to be in trans with the p.Tyr122Cys variant, both variants were re-classified as pathogenic and likely pathogenic, respectively. Conclusion: This report highlights the difficulty of identifying intermediate MSUD by newborn screening even including alloisoleucine determination, and the importance of functional studies in this age of molecular diagnostics.

\section{Incidental Finding of Mild Mucopolysaccharidosis Type li in a Child with a Diagnosis of Late-infantile Niemann-Pick C}

Sarah Donoghue ${ }^{1,2}$, Oliver Heath ${ }^{1}$, James Pitt ${ }^{2}$ and Maria Fuller ${ }^{3}$

${ }^{1}$ Department of Metabolic Medicine, Royal Children's Hospital, Melbourne, Australia, ${ }^{2}$ Department of Biochemical Genetics, Victorian Clinical Genetic Services, Melbourne, Australia and ${ }^{3}$ Department of Genetics and Molecular Pathology, SA Pathology, Adelaide, Australia

We report the case of a 6-year old boy with dual pathology who developed gelastic cataplexy the age of 2.5 years. He developed progressive ataxia and vertical supranuclear gaze palsy. He did not have organomegaly and he had a normal brain MRI. His investigations demonstrated an increased lysosphingomyelin 509 and molecular testing confirmed two variants in the NPC1 gene. The c.3562del (p.Glu1188Lysfs*54) was a known pathogenic variant and the other NPC1 variant c.3206T >C (p.Met1069Thr) was a missense variant in a moderately conserved region with a moderate histochemical difference between the amino acids. In-silico prediction software reported conflicting predictions of pathogenicity. An incidental finding of a novel hemizygous MPS II variant c.674A $>$ G (p.Tyr225Cys) was detected in the lysosomal gene panel. This variant was in a moderately conserved region and there was a large histochemical difference with the amino acid substitution. There have been previous reports of pathogenicity with a different amino acid substitution at the same position with a less significant histochemical change. In-silico prediction software predicted this variant to be deleterious. His urine total glycosaminoglycans were normal at $11.2 \mathrm{mg} / \mathrm{mmolCr}$ (RR 0-15.4). Urine mucopolysaccharide substrate analysis performed by mass spectrometry was abnormal with a result of $0.05 \mathrm{mmol} / \mathrm{molCr}$ $(\mathrm{RR}<0.03)$ and Iduronate-2-Sulfatase activity was low 4.0 (RR 23-80) consistent with a diagnosis of MPSII. There were mild features of dysplastic hips noted on his X-rays. Urine mucopolysaccharide substrate analysis performed by mass spectrometry is a more sensitive method than measurement of total glycosaminoglycans for detecting milder forms of MPSII.

\section{Late-onset Multiple ACYL-CoA Dehydrogenase Deficiency Identified by Newborn Screening}

Carolyn Foran ${ }^{1}$, Katrina Fisk ${ }^{2}$, Veronica Wiley ${ }^{3}$, Adviye Ayper Tolun ${ }^{1}$ and Shanti Balasubramaniam ${ }^{4}$

${ }^{1}$ NSW Biochemical Genetics Service, The Children's Hospital at Westmead, Sydney, Australia, ${ }^{2}$ Department of Molecular Genetics, The Children's Hospital at Westmead, Sydney, Australia, ${ }^{3}$ NSW Newborn Screening Program, The Children's Hospital at Westmead, Sydney, Australia and ${ }^{4}$ Genetic Metabolic Disorders Service, The Children's Hospital at Westmead, Sydney, Australia 
Background: Multiple acyl-CoA dehydrogenase deficiency (MADD), a rare autosomal recessive disorder of fatty acid and amino acid oxidation, is caused by a deficiency of electron transfer flavoprotein. Three broad clinical phenotypes exist: Type I (neonatal-onset with congenital anomalies), Type II (neonatal-onset without congenital anomalies) and Type III (mild/late-onset, often riboflavin responsive). Case report: A term female infant, was noted to have raised decanoylcarnitine (C10), tetradecanoylcarnitine (C14) and tetradecenoylcarnitine (C14:1), with borderline octanoylcarnitine (C8) on Newborn Screening. Her initial riboflavin level was low, 139 (NR: 174-471 nmol/L), and urine organic acid analysis was normal. Her initial plasma acylcarnitine profile showed increased hexanoylcarnitine (C6), C8, C10, decenoylcarnitine (C10:1), dodecanoylcarnitine (C12), C14 \& C14:1, possibly indicating riboflavin deficiency (neonatal and maternal), riboflavin transporter defect or MADD. Prior to commencing riboflavin supplementation, her level had normalized (200 nmol/L). Mother's riboflavin level, plasma acylcarnitine and urine organic acid analyses were normal, excluding maternal riboflavin deficiency. Riboflavin supplementation over a few months resulted in an improvement in her plasma acylcarnitine profile, although it did not completely normalize. Molecular testing identified compound heterozygous pathogenic variants in the ETFDH gene (NM_04453.4): c.[679C>T];[1234G >T] p.[(Pro227Ser)];[(Glu412*)]. Discussion: These findings are consistent with mild/late-onset MADD which is riboflavin-responsive. This case highlights the importance of Newborn Screening in identifying even the late-onset cases.

\section{Investigation into a Rare ACAD9 Variant of Unknown Significance Identified in Nonidentical Twins}

Simone Tregoning ${ }^{1,2}$, Alison G. Compton ${ }^{1,2,3}$, Charlotte L. Alston ${ }^{4}$, Robert W. Taylor ${ }^{4}$, David Coman ${ }^{5,6}$, Shalini Thirukeswaran ${ }^{1,2,3}$, Sumudu S. C. Amarasekera ${ }^{1,3}$,

Cas Simons $^{1,2}$ and David R. Thorburn ${ }^{1,2,3}$

${ }^{1}$ Murdoch Children's Research Institute, Melbourne, Australia, ${ }^{2}$ Victorian Clinical Genetic Services, Melbourne, Australia, ${ }^{3}$ Department of Paediatrics, University of Melbourne, Melbourne, Australia, ${ }^{4}$ Welcome Trust Centre for Mitochondrial Research, Institute for Cell and Molecular Bioscience Institute of Neuroscience, University of Newcastle upon Tyne, Newcastle upon Tyne, UK, ${ }^{5}$ Department of Metabolic Medicine, Royal Children's Hospital Brisbane, Brisbane, Australia and ${ }^{6}$ Discipline of Paediatrics, The Wesley-St Andrews Clinical School, University of Queensland, Brisbane, Australia

Background: ACAD9 is a highly conserved member of the mitochondrial acyl-CoA dehydrogenase protein family involved in mitochondrial Complex I assembly via binding of co-assembly factors NDUFAF1 and ECSIT. ACAD9 mutations cause isolated Complex I deficiency. Nonidentical twins with a biochemical diagnosis of Complex I deficiency were included in a cohort of patients lacking a molecular diagnosis after exome sequencing. Aim: To identify the cause of Complex I deficiency in a pair of nonidentical twins who were unsolved after exome sequencing. Methods: Investigations included exome sequencing, western blot analysis and linked-read (10X Genomics Chromium) whole genome sequencing. Results: A single heterozygous ACAD9 variant of unknown significance (VUS) was identified by exome sequencing in both sibs. Fibroblast western blotting showed that both twins displayed a characteristic 'ACAD9 deficiency signature' of decreased ECSIT and NDUFAF1 protein levels in fibroblasts, confirming that ACAD9 was likely the correct genetic diagnosis and that the rare, novel missense variant was pathogenic. However, a second mutation still remained to be identified. Further investigations were carried out using linked-read genome sequencing. Analysis of the
ACAD9 region identified a paired deletion-inversion structural rearrangement containing exon 2 and some of the surrounding intronic sequence. Conclusion: The shift towards high throughput sequencing technologies has changed the way laboratories are able to analyze and diagnose patients. For patients with VUS in known disease genes or in genes not previously linked to disease, the results of enzyme assays and other investigations are still needed to confirm pathogenicity.

\section{Diagnostic Odyssey in a Patient with Hyperphenylalaninemia (HPA)}

Beena Devanapalli ${ }^{1}$, Adviye Tolun ${ }^{1}$, Louisa Adams ${ }^{2}$, Sushil Bandodkar ${ }^{3}$ and Shanti Balasubramaniam²

${ }^{1}$ NSW Biochemical Genetics Service, Western Sydney Genetics Program, The Children's Hospital at Westmead, Westmead, Australia, ${ }^{2}$ Genetic Metabolic Disorders Service, Sydney Children's Hospital Network, Sydney, Australia and ${ }^{3}$ Biochemistry Department, The Children's Hospital at Westmead, Westmead, Australia

This case is about a 7 year old boy who was identified on newborn screening with HPA. Follow-up testing included dihydropteridine reductase (DHPR) activity on dried blood spot and urine pterins which were normal. He was diagnosed with mild PKU and managed with a protein restricted diet and PKU supplements. Upon relocating interstate at the age of 3 , further investigations were initiated in view of incongruous clinical presentation of severe developmental delay and autism spectrum disorder despite excellent dietary phenylalanine control. Investigations included plasma amino acid (PLAA), cerebrospinal (CSF) pterin and neurotransmitter analyses, molecular testing and brain MRI. PLAA analyses showed persistently increased phenylalanine levels ranging from $323 \mu \mathrm{mol} / \mathrm{L}(30-75 \mu \mathrm{mol} / \mathrm{L})$ on presentation to $172 \mu \mathrm{mol} / \mathrm{L}(31-90 \mu \mathrm{mol} / \mathrm{L})$ currently. Analysis of CSF pterins showed slightly elevated biopterin with a normal neopterin level. CSF neurotransmitter analysis showed decreased levels of homovanillic acid (HVA) with significantly reduced 5-hydroxyindoleacetic acid (5-HIAA). MRI of the brain also showed multiple small cystic lesions in the periventricular white matter suggesting gliosis, which was not typical of PKU. Molecular analyses of PAH and known HPA genes revealed no pathogenic sequence variation. Reanalysis of sequencing data performed after the 2017 description of DNAJC12-associated HPA identified a homozygous c.78+1del variant. Variants in this gene have been associated with mild nonBH4-deficient HPA (HPANBH4). The disorder is treatable with BH4 supplementation with or without L-DOPA/5-hydroxytryptophan and shows favourable response. Hence, HPANBH4 should be considered as a differential diagnosis in cases of mild HPA with normal pterins.

\section{Broadening the Phenotype-Genotype Correlation in VLCAD Deficiency: A Common ACADVL Variant in an Adult Diagnosed with Later Onset form}

Simone Coupe ${ }^{1}$, Beena Devanapalli ${ }^{1}$, Yusof Rahman ${ }^{2}$, Adviye Tolun ${ }^{1}$ and Sulekha Rajagopalan ${ }^{3}$

${ }^{1}$ NSW Biochemcal Genetics Laboratory, Sydney, Australia, ${ }^{2}$ Adult Genetic Medicine, Westmead Hospital, Sydney, Australia and ${ }^{3}$ SWS Clinical Genetics, Liverpool Hospital, Sydney, Australia

Very long-chain acyl-CoA dehydrogenase (VLCAD) is a mitochondrial enzyme that plays an important role in oxidation of long-chain fatty acids. VLCAD deficiency (OMIM \#201475) is an autosomal recessive disorder that has three forms: severe early 
onset characterized by dilated cardiomyopathy, with hypoketotic hypoglycemia; milder delayed onset induced by infections or long fasting with similar symptoms to early onset form; and the myopathic later onset form characterized by episodic symptoms of muscle cramps, myalgia, exercise intolerance and rhabdomyolysis. Initial diagnosis is an abnormal acylcarnitine profile in plasma. Specific enzyme testing in skin fibroblasts/lymphocytes and molecular analysis of the ACADVL are available for confirmation of the diagnosis. Case: A 59-year-old female presented with limb girdle myopathy, myalgia, exercise intolerance and high creatine kinase levels. She has been symptomatic from age 30. Plasma acylcarnitine analyses demonstrated increased C14, C14:1, C14:1/C10 and C14:1/C12:1, suggestive of VLCAD deficiency. Enzyme testing for VLCAD deficiency was performed on lymphocytes and showed only $16 \%$ activity compared to within run controls, confirming the diagnosis of VLCAD deficiency. The patient had a Neuromuscular gene panel (massively parallel sequencing) which showed the presence of a homozygous pathogenic variant in the ACADVL gene c. $848 \mathrm{~T}>$ C. This variant accounts for approx. $20 \%$ of pathogenic alleles detected by newborn screening and has been associated with a milder phenotype. Conclusion: This case illustrates the clinically heterogenous nature of this disorder and the challenge associated with diagnosis. It demonstrates the importance of enzyme testing coupled with molecular analysis to broaden the understanding of genotype-phenotype correlation.

\section{Presentation of Liver Function Abnormalities in Female Patients Diagnosed with OTC Deficiency in Victoria}

Sarah Donoghue ${ }^{1,2}$, Joy Lee Le $^{1,3}$ Mark Oliver ${ }^{3,4}$, Winita Hardikar ${ }^{3,4}$ and Heidi Peters ${ }^{1,3}$

${ }^{1}$ Department of Metabolic Medicine, The Royal Children's Hospital, Melbourne, Australia, ${ }^{2}$ Department of Biochemical Genetics, Victorian Clinical Genetic Services, Melbourne, Australia, ${ }^{3}$ Department of Paediatrics, University of Melbourne, Melbourne, Australia and ${ }^{4}$ Department of Gastroenterology, The Royal Children's Hospital, Melbourne, Australia

Background: Ornithine Transcarbamylase (OTC) Deficiency is an $\mathrm{X}$-linked condition that is the most prevalent urea cycle disorder. It is characterized by recurrent episodes of hyperammonemia. Females heterozygous for OTC deficiency have been reported to present with variable liver dysfunction, but this has been thought to be a relatively rare occurrence. Methods: We retrospectively reviewed the clinical records of female patients with OTC deficiency from January 2000 to July 2020 and identified patients who presented with acute liver dysfunction and coagulopathy or developed acute liver dysfunction following their diagnosis with OTC deficiency. Results: We identified 9/16 female OTC patients who had elevated liver transaminases associated with coagulopathy. Low serum albumin was present in 5/9 of patients during their acute liver dysfunction. Liver dysfunction with coagulopathy was present on presentation in 7/9 patients and 2/9 patients developed liver dysfunction in the context of metabolic instability associated with hyperammonemia. The degree of hyperammonemia at presentation did not correlate with the degree of liver dysfunction. Liver dysfunction and coagulopathy improved in most patients with long term treatment, however hepatic fibrosis was observed in the explanted livers of 3 children who underwent transplant. Conclusion: Liver dysfunction and coagulopathy in female OTC patients was present in over $50 \%$ of our cohort. The occurrence of liver dysfunction at presentation and during periods of metabolic instability with the resolution or partial resolution of abnormalities with treatment suggests that this liver injury may relate to the metabolic effects of OTC deficiency, lyonization or by the effect of secondary pathways.

\section{Clinical Untargeted Metabolomics and its Utility in Undiagnosed Cases: The First Case in Australia}

Adviye Ayper Tolun ${ }^{1}$, Ashley Hertzog ${ }^{1}$, Beena Devanapalli ${ }^{1}$, Zahrul Ismadi ${ }^{2}$, Gladys $\mathrm{Ho}^{3}$, Carolyn Ellaway ${ }^{2}$ and Alan $\mathrm{Ma}^{4}$

${ }^{1}$ NSW Biochemical Genetics Service, The Children's Hospital at Westmead, SCHN, Sydney, Australia, ${ }^{2}$ Genetic Metabolic Disorders Service, The Children's Hospital at Westmead, SCHN, Sydney, Australia, ${ }^{3}$ Department of Molecular Genetics, The Children's Hospital at Westmead, SCHN, Sydney, Australia and ${ }^{4}$ Clinical Genetics Department, The Children's Hospital at Westmead, SCHN, Sydney, Australia

Metabolomics is an 'omic' technology that is based on biochemical and molecular characterizations of the changes in metabolites due to genetic and environmental factors. Clinical untargeted metabolomics analysis has the potential to screen for dozens of inborn errors of metabolism (IEMs). A 12-year-old male with severe global developmental delay, multiple joint dislocations, bilateral congenital talipes, horseshoe kidney, visual impairment, epilepsy, poor growth and dairy intolerance/avoidance was evaluated in clinic. Extensive biochemical (including plasma and CSF amino acids, plasma acylcarnitines, targeted urine metabolic screen, urine organic acids, VLCFA, oligosaccharides and lysosomal enzyme studies) and genetic testing (including WES-trio analysis and microarray studies) were pursued with no clear diagnostic outcome. To further understand the underlying causes to this undiagnosed case, plasma and urine samples were submitted to Baylor Genetics, TX, USA for Global MAPS ${ }^{\circledR}$ untargeted metabolomics analysis. Elevation of pipecolate and reduction of several lysophospholipids, phosphatidylcholines, and plasmalogens suggested a possible peroxisomal disorder, as well as pyridoxine dependent seizures. Further detailed examination of the genes that are involved in the pathways suggested by the Global MAPS ${ }^{\circledast}$ is underway. Untargeted metabolomics analysis is increasingly being used in the screening and management of individuals with IEMs. This approach has the potential to expedite the diagnosis, as well as increasing the possibility to identify new biomarkers, new IEMs, and/or sources of phenotypic heterogeneity within a given disorder. However, it should be noted that in its current state this analysis should be considered as a supplementary test.

\section{Early-Onset Vitamin B6-dependent Epilepsy Due to PLPBP Mutations in a Premature Infant}

Oliver Heath ${ }^{1}$, James Pitt ${ }^{2,6}$, Simone Mandelstam ${ }^{3,6,7}$, Carl Kuschel $^{4}$, Anand Vasudevan $^{5}$ and Sarah Donoghue ${ }^{1,2}$

${ }^{1}$ Department of Metabolic Medicine, Royal Children's Hospital, Melbourne, Australia, ${ }^{2}$ Victorian Clinical Genetics Service, Murdoch Children's Research Institute, Melbourne, Australia, ${ }^{3}$ Department of Medical Imaging, The Royal Children's Hospital and Murdoch Children's Research Institute, Melbourne, Australia, ${ }^{4}$ Department of Obstetrics and Gynecology, The Royal Women's Hospital, Melbourne, Australia, ${ }^{5}$ Department of Genetics, The Royal Women's Hospital, Melbourne, Australia, ${ }^{6}$ Department of Paediatrics, University of Melbourne, Melbourne, Australia and ${ }^{7}$ Department of Radiology, University of Melbourne, Melbourne, Australia 
Background: Vitamin B6-dependent epilepsies are a consequence of decreased bioavailability of pyridoxal-5'-phosphate (PLP) which is a substrate for a large number of metabolic enzymes. Biallelic mutations in ALDH7A1 and PNPO are well-known causes of vitamin B6 dependent epilepsy and have characteristic biochemical abnormalities. In 2016, biallelic mutations in PLPBP were identified as a further cause of vitamin B6-dependent epilepsy. PLPBP encodes a binding protein that is integral in the maintenance of intracellular PLP. Aim: We describe a female patient with this disorder who was born at $34+4$ weeks gestation and developed tonic and generalized tonic-clonic seizures and hypoglycemia within four hours of life. Results: Empiric pyridoxine treatment was commenced and urine screening showed increased vanillactic, decreased creatine and normal $\Delta 1$-piperideine-6-caboxylate (P6C). PLP was substituted for pyridoxine as a result of these findings. Treatment resulted in cessation of seizures and near normalization of metabolite abnormalities. Whole exome sequencing showed the patient was compound heterozygous (c.207+1G $>\mathrm{T}$; p.(?)/c.722G $>\mathrm{A}$; p.(Arg241Gln)) for pathogenic mutations in PLPBP.Discussion: To date there appear to be no specific clinical or biochemical indicators of PLBP deficiency. However, prematurity, neonatal onset seizures and biochemical markers of deficient PLP-dependent enzymes appear to be important indicators for PLPBP genetic testing.

\section{Urinary Free Sialic Acid can be Increased Secondary to Streptococcus Pneumoniae Sepsis}

James Pitt ${ }^{1}$, Sarah Donoghue ${ }^{1,2}$, Oliver Heath ${ }^{2}$, Kai Mun Hong ${ }^{1}$ and Joel Smith ${ }^{3}$

${ }^{1}$ Victorian Clinical Genetics Services, Murdoch Children's Research Institute, Melbourne, Australia, ${ }^{2}$ Department of Metabolic Medicine, Royal Children's Hospital, Melbourne, Australia and 'Laboratory Services, Royal Children's Hospital, Melbourne, Australia

Urine free sialic acid (UFSA) is an important diagnostic biomarker for sialuria (GNE mutations) and infantile sialic acid storage disease/ Salla disease (SLC17A5 mutations). Traditionally, UFSA has been measured using specific single-plex methodology in relatively small cohorts of patients with clinical symptoms suggestive of these disorders. The use of multiplex tandem mass spectrometry urine screening (UMSMS) has meant that UFSA can be measured semiquantitatively in a much larger cohort of patients being investigated for suspected metabolic disorders. We report our experiences with three cases of increased UFSA secondary to Streptococcus pneumoniae sepsis. The three cases were ascertained during diagnostic evaluation after the children presented acutely unwell and inborn errors of metabolism were considered in the differential diagnosis. All three cases were found to have significantly increased UFSA with UMSMS screening. Additional testing ruled out genetic causes of increased UFSA in the first patient. All three patients had overwhelming sepsis with multiorgan dysfunction which was fatal. Abnormalities were demonstrated in glycosylation in the serum transferrin isoforms. We hypothesize that the neuraminidase of Streptococcus pneumoniae releases free sialic acid from endogenous sialylated glycoconjugates. Septic workup should be included in the investigation of patients with unexplained increases in UFSA.

\section{Simultaneous Determination of Total Homocysteine, Methionine, Methylmalonate and 2-Methylcitrate on DBS Samples by UPLC-MS/MS}

Mr Juan Carlos Velasquez ${ }^{1}$, Adviye Ayper Tolun ${ }^{1}$ and Beena Devanapalli ${ }^{1}$

${ }^{1}$ Children's Hospital at Westmead, Sydney, Australia
Elevations of total homocysteine (tHcy), methionine (Met), methylmalonic acid (MMA), and 2-methylcitrate (2MCA) are indicative of severe disorders including homocystinuria, hypermethioninemia, methylmalonic aciduria, cobalamin defects, and propionic acidemia. The aim of this study was to develop a rapid and accurate method to detect these analytes from dried blood spots (DBS) for monitoring patients to allow for prompt therapy adjustment. Method: The chromatographic separation was achieved at room temperature on a reversed-phase high strength silica column (ACQUITY UPLC ${ }^{\circledR}$ HSS T3, $1.8 \mathrm{um}, 2.1 \times 150 \mathrm{~mm}$ ), using a gradient program (mobile phase A: $0.4 \%$ formic acid in MilliQ water and B: acetonitrile and methanol $(50: 50, \mathrm{v} / \mathrm{v}))$. Stable isotope-labeled internal standards were used for quantification. The compounds were analysed by multiple-reaction monitoring in negative (MMA and 2MCA) and positive (tHcy and Met) ionization modes. Total analysis time per sample was $6 \mathrm{~min}$. Results: The calibration curve for each analyte showed good linearity (avg r2 $\geq 0.993$ ). The intra- and inter-assay imprecision varied between $8 \%-19 \%$ for all compounds assayed, with MMA resulting in highest imprecision. The recovery of analytes was between $80 \%$ and $126 \%$. Reference ranges were determined using DBS samples prepared from blood that was received for routine biochemical genetic investigations. Analysis of DBS samples from CDC newborn screening quality assurance program showed a good correlation for all these analytes (avg r2 $\geq 0.997$ ). Conclusion: A simple and accurate UPLC-MS/MS method for the simultaneous determination of tHcy, Met, MMA, 2MCA from DBS samples has been developed and validated.

\section{Improving the Diagnostic Efficiency of Fabry Disease: Clinical and Biochemical Approach}

Yuka Kong ${ }^{1,2}$, Jennifer Saville ${ }^{2}$, Corey Markus ${ }^{2}$, Janice Fletcher ${ }^{1,2}$ and Maria Fuller ${ }^{1,2}$

${ }^{1}$ The University of Adelaide, Adelaide, Australia and ${ }^{2}$ SA Pathology, Adelaide, Australia

Fabry disease (FD) is an X-linked inherited metabolic disorder caused by variants in the alpha-galactosidase A (GLA) gene and these variants result in a deficiency of the lysosomal enzyme with a consequent pathological accumulation of glycosphingolipids in multiple organs. Due to nonspecific symptoms and variability in X-chromosome inactivation patterns, diagnosis is particularly challenging for later-onset disease and heterozygotes. There is considerable overlap between the enzyme activity of heterozygotes and unaffected patients, making identification of Fabry patients difficult. Therefore, a greater understanding of the biochemistry behind FD is urgently needed to refine current diagnostic algorithms. We proposed to improve the sensitivity of the diagnosis of FD by establishing new reference intervals for alpha-Galactosidase activity in dried blood spots, refining the existing lyso-CTH method and measuring sphingolipids of interest to create an enhanced diagnostic algorithm. We analyzed 2661 dried blood spots using the log ratio of alpha:beta galactosidase enzyme activity, using a three component Gaussian mixture model. We showed clear separation between classical Fabry patients and unaffected patients. Some overlap in distributions was observed between heterozygotes and unaffected patients. This newly determined log ratio may improve the discrimination of heterozygotes. Analysis of 97 plasma glycosphingolipids revealed 11 had significantly greater abundance in Fabry disease $(N=20)$ when compared to controls $(N=60)$. In particular, ceramide trihexoside $(16: 0$, 18:0, 22:0, 24:0, 24:1) and hydroxylated trihexosylceramide (16:0 and 24:0). Similarly, lyso-CTH and its analogues also showed higher 
concentrations in Fabry patients. These results suggest the possibility for enhancement of diagnosis of Fabry disease.

\section{CANCER GENETICS}

\section{Mapping Cancer Transcriptomes with Long-Read Sequencing}

Stephen Kazakoff ${ }^{1}$, Pamela Mukhopadhyay ${ }^{1}$, Futoshi Kawamata ${ }^{2}$, Catherine Bond ${ }^{1}$, Katia Nones ${ }^{1}$, Akinobu Takeomi ${ }^{2}$, Vicki Whitehall ${ }^{1,3,4}$ and Ann-Marie Patch ${ }^{1,3}$

${ }^{1}$ QIMR Berghofer Medical Research Institute, Herston, Australia, ${ }^{2}$ Hokkaido University Graduate School of Medicine, Sapporo, Japan, ${ }^{3}$ The University of Queensland, Brisbane, Australia and ${ }^{4}$ Pathology Queensland, Brisbane, Australia

Dysregulation of gene expression in cancer may confer a selective advantage and affect tumor treatment response. Although short-read RNASeq can provide quantitative expression data, it is limited in capacity to resolve complex isoforms whereas emerging long-read technologies can sequence full-length transcripts. Our aim was to profile cancer transcriptomes to explore cancer-specific transcript characteristics. We used the PacBio RS II and Sequel II platforms to generate long reads from RNA from primary cancer, metastasis and normal colon tissue samples from four colorectal cancer (CRC) patients. Transcript variation was assessed by comparison of splice junctions in cancer and normal datasets and verified using RNA-Seq data. An average of 31,000 and 5,000 fulllength transcripts were identified per sample using the Sequel II and RS II platforms, respectively. By comparing the RS II data to the GENCODE v31 gene model, we found an average of $73 \%$ of transcripts contained annotated splice junctions and approximately $25 \%$ of transcripts had unannotated splice boundaries. A novel spliced transcript was also identified that was detectable in $>10 \%$ of TCGA CRC, breast, endometrial and oesophageal samples. Transcripts generated using the Sequel II platform also revealed allele-specific expression and somatic driver mutation expression. We show that long-read cancer RNA sequencing can identify cancer driver mutation expression, allele-specific expression and novel cancer-specific transcripts. We also show that publicly available RNASeq data can be used to verify and identify the cohort frequency of these events. Further work could support somatic transcript splice junctions as disease biomarkers.

\section{Investigation of Mismatch Repair Gene Branchpoints}

Daffodil Canson ${ }^{1,2}$, Troy Dumenil ${ }^{1}$, Michael Parsons ${ }^{1}$, Dylan Glubb ${ }^{1,2}$ and Amanda Spurdle ${ }^{1,2}$ ${ }^{1}$ QIMR Berghofer Medical Research Institute, Brisbane, Australia and ${ }^{2}$ Faculty of Medicine, The University of Queensland, Brisbane, Australia

Recent large-scale studies have generated genomewide annotations of experimentally inferred and predicted branchpoints (BPs), with most BPs identified by genomic coordinates. We conducted an investigation of mismatch repair (MMR) gene BP motifs to assess the likely clinical impact of variation within them. Genomic BP coordinates for MLH1, MSH2, MSH6 and PMS2 were obtained from three large-scale experimental datasets, and two predicted BP datasets generated by the Branchpointer and Branchpoint Prediction (BPP) tools. Experimentally inferred and predicted BPs were manually annotated and mapped for each MMR gene intron. Variants colocalized with the BP motif were identified from the ClinVar and gnomAD public databases. Selected minigene assays were conducted to assess functional impact of variation at highly conserved positions in the BP motif: the BP nucleotide and two nucleotides upstream of the BP (i.e., BP-2nt). We annotated 125 experimentally inferred and predicted BPs in MMR genes. BP redundancy was observed in most introns. Prediction sensitivity was low for Branchpointer (31\%) and BPP (20\%). Minigene assay results showed the BP-2nt to be more important to BP motif integrity than the BP nucleotide itself. Variation at the conserved BP motif positions was more common, providing evidence against pathogenicity, for introns with BP redundancy. Our results suggest that experimental data is critical to generate comprehensive maps of BP motifs. Nevertheless, for MMR genes, $\mathrm{BP}$ redundancy in most introns suggests that there will be few high-risk alleles that impact on function via BP site usage.

\section{Dicentric Chromosomes as a Major Driver of Gene Loss in Cancer: Evidence from two Recurrent Dicentric Chromosomes Produced by Different Mechanisms}

Ruth N. MacKinnon ${ }^{1,2}$ and Joanne S. White ${ }^{2}$

${ }^{1}$ Victorian Cancer Cytogenetics Service, St Vincent's Hospital, Melbourne, Australia and ${ }^{2}$ Department of Medicine (St Vincent's), University of Melbourne, Melbourne, Australia

Unbalanced translocations can cause tumor suppressor gene loss. We demonstrated that the incidence of dicentric chromosomes in myeloid malignancy is underestimated, as many are cryptic. Some dicentrics lose a centromere. Some are functionally monocentric - having closely spaced centromeres or one inactivate centromere. All of these cryptic dicentrics appear monocentric. Dicentric chromosome formation only requires one random, selectable event: (1) telomere fusion (where chromosome instability causes copy number aberration) or (2) a reciprocal exchange producing a dicentric and an acentric product. Our hypothesis is that monocentric unbalanced translocations are uncommon because they require two random events (translocation and chromosome loss). We therefore predict that most unbalanced translocations are dicentric. We present two contrasting recurrent dicentrics in myeloid malignancy that support this. 20q12 loss is a recurrent aberration. In our published series of unbalanced chromosome 20 translocations, all were dicentric, formed by either mechanism. We demonstrated telomere fusion (identifying residual telomere sequence) in the recurrent $\operatorname{dic}(20 ; 22)$ (mechanism 1). We have now studied thirteen examples of the recurrent unbalanced 5;17 translocation. All but one were dicentric. The single exception arose via two steps. The first was a balanced $t(5 ; 17)$, present in some cells. The second step, the selectable event, was loss of the $\operatorname{der}(5)$ which carried TP53 and the $5 \mathrm{q}$ tumor suppressor gene region. In human genome studies, centromeres have largely remained a mystery. New sequencing technologies are beginning to address this. Centromeres are rarely identified in rearranged chromosomes, however, as our study demonstrates, they may hold important clues to understanding cancer.

\section{Implementation of a New Targeted Next Generation Sequencing Assay Assisting Comprehensive Genomic Profiling of Solid Tumors for the Most Study}

Julia R. Dobbins ${ }^{1,4}$, John Grady ${ }^{3}$, Rosalie Kenyon ${ }^{1,4}$, Rob King ${ }^{1,4}$, Anna L. Brown ${ }^{1,4}$, Michael P. Brown ${ }^{2}$, Eryn Dow ${ }^{2}$, Rachael Chang ${ }^{2}$, Anne Milton ${ }^{2}$, Melinda Whelan ${ }^{2}$, Deirdre Russo ${ }^{2}$, Keith Thornton ${ }^{3}$, Tharindi Kalutantiri-Ip ${ }^{3}$, Mandy L. Ballinger ${ }^{3}$, David M. Thomas ${ }^{3}$ and Hamish S. Scott ${ }^{1,4}$

${ }^{1}$ Centre for Cancer Biology, Adelaide, Australia, ${ }^{2}$ Cancer Clinical Trials Unit, Royal Adelaide Hospital, Adelaide, Australia, ${ }^{3} \mathrm{G}$ arvan Institute of Medical Research, Darlinghurst, Australia and ${ }^{4}$ SA Pathology, Genetics and Molecular Pathology, Adelaide, Australia

The Molecular Screening and Therapeutics study (MoST), run under the auspices of Omico, a network of research institutes, pathology 
providers and industry partners nationwide, delivers precision medicine to patients enrolled with rare, advanced or incurable cancers through comprehensive genomic profiling of tumors, identification of clinically actionable genetic lesions and biomarkers, and subsequently cancer precision therapies targeting these specific molecular lesions. In partnership with Omico, the South Australian team comprizing the Royal Adelaide Hospital (RAH) clinical trials unit and SA Pathology service providers including Genetics and Molecular Pathology (GMP) research, diagnostics and sequencing facilities, have supported the MoST study, providing molecular screening for patients enrolled through the study. We have implemented a new sequencing platform, the TruSight Oncology 500 assay (TSO500, Illumina), which targets 523 genes analyzed from DNA and RNA, identifies genetic lesions, performs copy number variant calling, novel fusion detection and MSI and TMB determination. Since implementing the TSO500 assay in September 2019, molecular sequencing for 240 patients has been performed, identifying clinically actionable molecular lesions which assist recommendations for therapeutic intervention. Of these, 68 were South Australian patients enrolled through the Adelaide MoST substudy. Approximately 50\% of these SA patients had clinically actionable variants identified (including mutations, amplifications, deletions and novel fusions in genes such as BRCA1/2, ERBB2, IDH1/2, KIT, PIK3CA, MAPK, and FGFR1/3/4, and also high $\mathrm{TMB} / \mathrm{MSI}$ ) resulting in therapy recommendations and enabling access to experimental therapies. Implementing the TSO500 assay has advanced clinical testing of tumors and will ultimately result in improved patient outcomes.

\section{CLINICAL GENETICS}

\section{A Rare Cause of Hyperlipidemia Identified by Genomic Testing}

Sharmila Kiss ${ }^{1}$, Joy Yaplito-Lee ${ }^{1,3}$, James Pitt ${ }^{2,3}$, Duncan MacGregor ${ }^{4}$, Jane Wallace ${ }^{2}$, Melanie Marty ${ }^{2}$ and Natasha Brown ${ }^{2,3}$

${ }^{1}$ The Royal Children's Hospital, Melbourne, Australia, ${ }^{2}$ Victorian Clinical Genetics Services, Murdoch Children's Research Institute, Melbourne, Australia, ${ }^{3}$ Department of Paediatrics, University of Melbourne, Melbourne, Australia and ${ }^{4}$ Department of Anatomical Pathology, The Royal Children's Hospital, Melbourne, Australia

Background: Sitosterolemia is an extremely rare, autosomal recessive disease characterized by accumulation of plant sterols in blood and tissues. It is caused by homozygous or compound heterozygous variants in either ABCG5 or ABCG8, which encode the subunits of sterolin, the sterol efflux transporter that pumps sterols out to the intestinal lumen or into bile. Loss of function of this transporter leads to increased intestinal absorption and decreased biliary excretion of all dietary sterols and thus to progressive accumulation of sterols. There is clinical variability in the presentation of individuals with sitosterolemia, from asymptomatic to early lethality. Clinical features may include xanthoma, arthritis, thyroid dysfunction, premature atherosclerotic disease, splenomegaly and hematologic manifestations including unexplained hemolytic anemia, macrothrombocytopenia and abnormal bleeding. Results: We report an 8-year-old girl who presented with a 2.5 year history of multiple xanthomas. In our case, the correct diagnosis was delayed for more than 2 years after initial presentation and she was treated for familial hypercholesterolemia with poor response for a year. Conclusion: The mainstay of therapy for sitosterolemia is dietary restriction of both cholesterol and plant sterols (vegetable oils, chocolate, margarine, avocado, nuts and seeds) and the use of ezetimibe (sterol absorption inhibitor). The patient was tried on a low plant sterol and cholesterol diet for 6 weeks with insignificant response in lipid profile and therefore ezetimibe at $10 \mathrm{mg}$ daily was added. After initiation of treatment, there was marked improvement in her cutaneous lesions, total cholesterol and sitosterol levels.

\section{Developing a Quality of Life Measure for Patients with Hereditary Spastic Paraplegia}

Sue-Faye Siow ${ }^{1,2,3}$, Jane Fleming ${ }^{2,4}$, Kristine Barlow-Stewart ${ }^{4}$ and Carolyn Sue ${ }^{1,4,5}$

${ }^{1}$ Northern Clinical School, University of Sydney, Sydney, Australia, ${ }^{2}$ Department of Clinical Genetics, Royal North Shore Hospital, Sydney, Australia, ${ }^{3}$ Department of Clinical Genetics, Westmead Hospital, Sydney, Australia, ${ }^{4}$ Sydney Medical School, University of Sydney, Sydney, Australia and ${ }^{5}$ Department of Neurology, Royal North Shore Hospital, Sydney, Australia

Background: Hereditary spastic paraplegia (HSP) is a neurodegenerative condition associated with lower limb spasticity and weakness. Patients with HSP have reported reduced quality of life (QoL) indices when compared to controls. There are no disease-specific QoL questionnaires for HSP to identify aspects of care most important to patients. Aim: To design an HSP-specific QoL questionnaire for use as a patient reported outcome measure (PROM) in clinic and for research. Methods: A list of potential HSP-specific QoL questions was identified from a recent literature review. These were refined by a 12-member expert panel including patients, neurologists, a carer, clinical nurse specialist, and patient support group representative, using a 2 -step modified Delphi process. An $80 \%$ consensus was the cut-off for modifying or including a question. Results: For the first round, the $24 \mathrm{HSP}$-specific QoL questions were grouped into $5 \mathrm{sec}$ tions for expert evaluation. Of these, 9 modified questions and 1 original question met the $80 \%$ consensus cut-off in the final round including: 4 items for 'Symptoms specific to HSP', 3 items for 'Genetic nature of HSP', and 1 item each for 'Progressive nature of HSP', 'Visibility of HSP', and 'Access to health care'. Discussion/Conclusion: In summary, we have designed a 10-item HSP-specific QoL questionnaire for use in piloting with a cohort of HSP patients. We aim to use the final survey as a PROM for use in standard clinical practice and future clinical trials; to inform identification of physical and psychological distress and facilitate a better health outcome for patients.

\section{The Core Outcome Development for Carrier Screening (CODECS) Study: Systematic Review of Outcomes in Studies Implementing Reproductive Genetic Carrier Screening}

Ebony Richardson ${ }^{1}$, Alison McEwen ${ }^{1}$, Toby Newton-John ${ }^{1}$, Karine Manera ${ }^{2}$, Ashley Crook ${ }^{1}$ and Chris Jacobs ${ }^{1}$

${ }^{1}$ Graduate School of Health, University of Technology Sydney, Sydney, Australia and ${ }^{2}$ Sydney School of Public Health, University of Sydney, Sydney, Australia

Background: A lack of consensus for 'what to measure' can result in different approaches to research on the same topic, termed outcome heterogeneity, which has implications for the quality and comparability of studies. Reproductive genetic carrier screening (RGCS) is one area of practice where outcome heterogeneity is observed, leading to difficulties in identifying high-quality research evidence to support its implementation into clinical practice. Aim: To conduct a systematic review to identify study designs, outcomes, and 
measures in studies implementing RGCS. Methods: MEDLINE, CINAHL, PsycINFO, and EMBASE were searched for peerreviewed, published studies that have offered RGCS. Outcomes, and where supplied, their definition, method of measurement and time point, were extracted. Results: Over 140 outcomes were measured across 53 included studies, ranging from 2-20 outcomes per study with a mean of 7 , indicating significant outcome heterogeneity. We identified diverse methods of measurement for the same or similar outcomes. Conclusions: Core outcome sets, an agreed minimum set of outcomes that should be measured and reported in all studies on a particular topic, are widely adopted across the medical field to address issues with outcomes in research. This systematic review is the first step in the development of a core outcome set for RGCS. Our results indicate that the development of a core outcome set may help to address outcome heterogeneity in this area. Further stages are underway and include a systematic review of the qualitative literature, semi-structured focus groups/interviews with patients, and a Delphi survey and consensus meeting with key stakeholders.

\section{Rapid Whole Exome Sequencing Identifies the Cause of a Fatal Neonatal Cardiomyopathy}

Noha Elserafy ${ }^{1,2}$, Sulekha Rajagopalan ${ }^{1,2}$, Morgan Rice ${ }^{1}$, Doron Shein ${ }^{1}$, Jonathan Forsey ${ }^{3}$, Michael Buckley ${ }^{4}$ and Tony Roscioli4,5

${ }^{1}$ Liverpool Hospital, Sydney, Australia, ${ }^{2}$ The University of New South Wales, Sydney, Australia, 3'Sydney Children's Hospital Network, The Children's Hospital at Westmead, Sydney, Australia, ${ }^{4}$ Randwick Genetics Laboratory, NSW Health Pathology, Sydney, Australia and ${ }^{5}$ Sydney Children's Hospital Randwick, Sydney, Australia

Pediatric cardiomyopathies are rare but serious disorders of the heart muscle. The age of onset ranges from the prenatal period, infancy or during childhood. Prognosis is usually poor with high morbidity and mortality. Some children will require a cardiac transplantation. We present a female infant with cardiomyopathy and hydrops, who was born to consanguineous parents. Rapid trio Whole Exome Sequencing (WES) identified likely pathogenic variants in the ALPK3 gene. This gene is linked to autosomal recessive cardiomyopathy with variable occurrence of extra-cardiac features. Case: Antenatal scan at $32+3 / 40$ week gestation showed fetal cardiac hypertrophy with impaired myocardial contractility, moderate ascites, pericardial and pleural effusions. She was born via planned cesarean section at 33/40 weeks. She required respiratory support and anti-failure medications to support her cardiac function with minimal effect. Echocardiogram demonstrated biventricular hypertrophy and left ventricular non-compaction with poor systolic function. Dysmorphic facial features were also noted. Rapid WES was arranged on Day 4 of life and results were available on Day 24. She had a missense homozygous likely pathogenic ALPK3 variant. The parents were confirmed carriers. Heterozygotes of pathogenic ALPK3 variants are also at risk of developing dilated/hypertrophic cardiomyopathy. Hence, cardiac screening for the parents and genetic testing for the baby's older siblings was offered. Conclusion: This case demonstrates the utility and benefit of rapid WES in reaching a timely diagnosis for a critically ill neonate. The early genetic diagnosis assisted in clinical decision-making. It also helped to identify at-risk family members who need cardiac surveillance.

\section{Compound Heterozygous ATP2B1 Variants as a Likely Cause of a Novel Neurodevelopmental Malformation Syndrome with Persistent Hypocalcemia}

Patrick Yap ${ }^{1,2}$, Polona Le Quesne Stabej ${ }^{2}$, Lisa G. Riley ${ }^{3,4}$, Adam Bournazos ${ }^{4,5}$, Purvi M. Kakadiyaa ${ }^{2,6}$, Stefan K. Bohlander ${ }^{2,6}$, Ben Curran ${ }^{2}$, Salam Alburaiky ${ }^{1}$, Ian Hayes ${ }^{1}$, Sandra T. Cooper ${ }^{4,5,7}$ and Cristin Print ${ }^{2}$

${ }^{1}$ Genetic Health Service New Zealand, Grafton, New Zealand, ${ }^{2}$ Department of Molecular Medicine and Pathology, University of Auckland, Auckland, Grafton, New Zealand, ${ }^{3}$ Rare Diseases Functional Genomics, Kids Research, The Children's Hospital at Westmead and The Children's Medical Research Institute, Sydney, Australia, ${ }^{4}$ Discipline of Child \& Adolescent Health, Sydney Medical School, University of Sydney, Sydney, Australia, ${ }^{5}$ Kids Neuroscience Centre, Kids Research, Children's Hospital at Westmead, Sydney, Australia, ${ }^{6}$ Leukaemia and Blood Cancer Research Unit, Department of Molecular Medicine and Pathology, University of Auckland, Grafton, New Zealand and ${ }^{7}$ The Children's Medical Research Institute, 214 Hawkesbury Road, Westmead, Sydney, Australia

We describe a male proband born to nonconsanguineous European couple, phenotypically characterized by global developmental delay, mild intellectual disability, postnatal-onset asymmetrical growth restriction, Pierre-Robin sequence, talipes equinovarus, pectus carinatum, and brachysyndactyly. Brain MRI showed nodular subependymal heterotopia within the left lateral ventricle. Brachymesophalangy type A2/A4 with 4/5-toe syndactyly are rarely observed in known syndromes though a salient feature of Feingold syndrome. Microcephaly and gastrointestinal atresia are absent. Sequencing of MYCN and HOXD13 was negative. The proband has persistent mild hypocalcemia and hyperphosphatemia. The mother has intermittent hypocalcemia. Parathyroid hormone levels are normal in both. Proband-parents exome sequencing identified a maternally inherited ATP2B1 splice-site (NM_001682.2: c. $3060+2 \mathrm{~T}>\mathrm{G})$ and a paternally-inherited missense variant (NM_001682.2:c.2938G>T; p.(Val980Leu)). ATP2B1 encodes plasma membrane calcium-transporting ATPase-1, involved in $\mathrm{Ca} 2+$ homeostasis with high expression in brain. Homozygous ablation of Atp2b1 in mice is embryonic lethal. ATP2B1 is intolerant to truncating $(\mathrm{pLI}=1)$ and missense variations $(z=5.29)$. PCR of cDNA synthesized from fibroblast-derived mRNA establishes that the c. $3060+2 \mathrm{~T}>\mathrm{G}$ variant induces mis-splicing with undetectable levels of normal splicing. All normally spliced ATP2B1 mRNA contains the c.2938G>T;p.(Val980Leu) variant. Val980 lies in a transmembrane domain and is highly conserved from mammals to yeast. Western blot of fibroblast lysate shows ATP2B1 levels are reduced to $\sim 20 \%$ of control, indicating reduced stability in the p.(Val980Leu) variant. The compound heterozygous ATP2B1 variants are the likely cause for the described phenotype. We seek additional cases with recessive variants in ATP2B1 to confirm and extend the phenotypic spectrum of this proposed novel disorder.

\section{Nationwide Diagnostic Utility of Clinical Genomics in Patients with Suspected Genetic Kidney Disease}

Kushani Jayasinghe ${ }^{1,2,3}$, Zornitza Stark ${ }^{4,7}$, Peter Kerr ${ }^{1,2}$, Catherine Quinlan ${ }^{3,5}$, Andrew Mallett ${ }^{5,7}$ and Renal Genetics Flagships, KidGen collaborative ${ }^{7,8}$

${ }^{1}$ Monash Health, Melbourne, Australia, ${ }^{2}$ Monash University, Melbourne, Australia, ${ }^{3}$ Murdoch Children's Research Institute, Melbourne, Australia, ${ }^{4}$ Victorian Clinical Genetics Services, Melbourne, Australia, ${ }^{5}$ Genetic Health Queensland, Royal Brisbane and Women's Hospital, Brisbane, Australia, ${ }^{6}$ Department of Paediatric Nephrology, Royal Children's Hospital, Melbourne, 
Australia, ${ }^{7}$ Australian Genomics Health Alliance, Melbourne, Australia and ${ }^{8}$ Melbourne Genomics Health Alliance, Melbourne, Australia

Background: With increased understanding of genetic kidney disease (GKD), genomic testing is translating from research to clinic. Rigorous evaluation of clinical practice and patient outcomes is required to guide value-based healthcare. Aim: To describe diagnostic outcomes of clinically accredited genomic testing delivered by nationwide multidisciplinary clinics for patients with suspected GKD. Methods: Sequential incident patients undergoing clinically indicated genomic testing for presumed GKD from 18 Australian MDT clinics 2016-19 were analyzed (HREC/16/MH/251). A molecular diagnosis constituted clinical reporting of pathogenic and/or likely pathogenic variant/s in gene/s associated with the patient's kidney phenotype with concordant inheritance. All genomic testing included restriction of variant analysis to a phenotype-derived gene list. Results: 1088 patients underwent genomic testing between $2016-2019.48 \%$ of the cohort were male, median age at the time of genomic test was 30 years (range <1year to 81). 491 patients $(45 \%)$ received a positive diagnostic on genomic sequencing. Of these, 71/448(16\%) had reclassification of their original diagnosis, $176 / 448(39 \%)$ had clarification of their diagnosis, and $187 / 448(42 \%)$ had confirmation of the suspected diagnosis following genomic testing. In addition, $14 / 448(3 \%)$ patients with a previous unknown cause of renal failure received a diagnosis. The diagnostic yield was highest in infants $<1$ year of age $(68 \%)$ and lowest $(37 \%)$ in those aged above 50 (OR 3.6, 95\% CI [19.1, 6.72], $p<.001$ ), for infants compared to age $>50$ at time of test). Conclusions: In this nationwide, pragmatic cohort, genomic testing demonstrated substantial diagnostic utility, especially in children and young adults. Clinical utility studies are required to clarify impact of these diagnostic outcomes.

\section{Clonal Hematopoiesis as a Natural Functional Assay of TP53 Germline Variant Pathogenicity}

Cristina Fortuno ${ }^{1}$, Tina Pesaran ${ }^{2}$, Jill Dolinsky ${ }^{2}$, Kelly McGoldrick ${ }^{2}$, Paul A. James ${ }^{3}$ and Amanda B. Spurdle ${ }^{1}$

${ }^{1}$ QIMR Berghofer Medical Research Institute, Brisbane, Australia, ${ }^{2}$ Ambry Genetics, Aliso Viejo, USA and ${ }^{3}$ Parkville Familial Cancer Centre, Peter MacCallum Cancer Centre and Royal Melbourne Hospital, Melbourne, Australia

Approximately $30 \%$ of TP53 variants identified in the blood of patients undergoing genetic testing for hereditary cancer are suspected to have somatic origin, mostly due to clonal hematopoiesis $(\mathrm{CH})$, and this proportion increases with lower variant allele fraction (VAF). Positive selection has been proposed to be a mechanism driving $\mathrm{CH}$, with somatic variants providing fitness advantage to a subset of blood cells. For this reason, we hypothesized that TP53 variants with low VAF are pathogenic drivers of $\mathrm{CH}$. If true, low VAF status could be used a positive predictor of TP53 variant pathogenicity. We compared VAF between 1069 TP53 variants classified as (likely) pathogenic and 35327 TP53 variants classified as (likely) benign using data from Ambry Genetics. VAF distribution was strikingly different between the groups: benign variants showed an expected normal distribution around 50:50 ratio (average 48.1, standard deviation 3.7), while pathogenic variants showed a bimodal distribution. We then compared the proportion of pathogenic and benign variants observed in different VAF bins, and determined that $\mathrm{VAF}<33.22$ provided very strong evidence towards pathogenicity (likelihood ratio $>350: 1$ ). Further, we found that this evidence type could be applied to approximately 5\% of unique TP53 germline variants of uncertain significance present in the same dataset. This data confirms that $\mathrm{CH}$ is a distinctive characteristic of pathogenic TP53 variants, and provides a new interpretation of this phenomenon in that it can be used as a natural functional assay to assist with the interpretation of TP53 germline variants.

\section{Chedda Syndrome is an Underrecognized Neurodevelopmental Disorder with a Highly Restricted ATN1 Mutation Spectrum}

Elizabeth Palmer ${ }^{1}$, Mais O Hashem ${ }^{3}$, R. D. Clark ${ }^{4}$ Subha Ramanathan ${ }^{4}$, Lois Starr ${ }^{5}$, Danita Velasco ${ }^{5}$, John Karl De Dios ${ }^{6}$, Waafa Eyaid ${ }^{7}$, Emily Singh ${ }^{8}$, Kirsty McWalter ${ }^{9}$, Valerie Cormier-Daire ${ }^{10}$, Maya Chopra ${ }^{10}$, Karin Panzer ${ }^{11}$, Alpa Sidhu ${ }^{11}$, Tracy DuddingByth $^{12}$ and Fowzan Alkuraya ${ }^{3}$

${ }^{1}$ Sydney Children's Hospital Network, Sydney, Australia, ${ }^{2}$ School of Women's and Children's Health, University of New South Wales, Sydney, Australia, ${ }^{3}$ King Faisal Specialist Hospital and Research Centre, Riyadh, Saudi Arabia, ${ }^{4}$ Loma Linda University School of Medicine, Division of Medical Genetics, Loma Linda, USA, ${ }^{5}$ Munroe-Meyer Institute, Omaho, USA, ${ }^{6}$ Dayton Children's Hospital, Dayton, USA, ${ }^{7}$ King Abdullah Specialized Children's Hospital, Riyadh, Saudi Arabia, ${ }^{8}$ Department of Pediatrics, Medical College of Wisconsin, Milwaukee, USA, ${ }^{9}$ GeneDx, Gaithersburg, USA, ${ }^{10}$ Université de Paris, Hôpital Necker-Enfants Malades, Institut IMAGINE, Paris, France, ${ }^{11}$ University of lowa Hospitals and Clinics, lowa City, USA and ${ }^{12}$ Genetics of Learning Disability Service, Newcastle, Australia

We describe the clinical features of six unrelated individuals with de novo, rare, previously unreported missense or in-frame deletions/ duplications within the 'HX motif of exon 7 of $A T N 1$. We previously proposed that individuals with de novo variants in this region of ATN1 should be considered as being affected by the syndromic condition Congenital Hypotonia, Epilepsy, Developmental delay, and Digital Anomalies (CHEDDA: MIM 618494), which is distinct from Dentatorubral-Pallidoluysian Atrophy (DRPLA: MIM 125370), secondary to expansion variants in exon 5 of ATN1. We confirm that there are universal recognizable phenotypic features of CHEDDA, including distinctive facial features, global developmental delay and infantile hypotonia. Developmental delay is highly variable and can be surprizingly mild. Abnormalities of palmar creases as well as overlapping toes and fingers are common, and individuals at the more extreme end of the spectrum can fit an arthrogryposis picture. Common comorbidities include severe feeding difficulties, often requiring gastrostomy support, due to a combination of low tone, gastroesophageal reflux and dysphagia, as well as visual and hearing impairments. Epilepsy and congenital malformations of the brain, heart and genitourinary systems are frequent but not universal. Our study confirms the clinical entity of CHEDDA secondary to a highly restricted mutational signature of missense and indel variants within 12 amino acid region of ATN1. We propose a clinical schedule for screening, surveillance and early intervention emphasizing the utility of neuroimaging for prognostication of the severity of neurological manifestations.

\section{Development and Experience of a Combined Genetic Dermatology Clinic in Western Australia}

Cathryn Poulton ${ }^{1}$

${ }^{1}$ GSWA, Perth, Australia

Background: The number of known genodermatosis has risen rapidly since 1992 and with this, our understanding of the molecular basis of both single gene and mosaic conditions has progressed. Being able to accurately phenotype patients is essential to guide genetic testing and 
Abstracts for the Human Genetics Society of Australasia Virtual Conference 24-25 November 2020

interpret results. The setting up of a multidisciplinary clinic enables the clinical geneticist and dermatologist to see the families in the same appointment and maximize use of time, resources and expertise and optimize patient care. Aim: To describe the cohort of patients attending a combined genetic-dermatology clinic and insights gained. Methods: We performed a retrospective chart review and database exploration of patients seen in combined genetic dermatology clinic during 5 years at Princess Margaret Hospital. We reviewed data on demographics, referral source, initial diagnosis, investigations performed including uptake of genetic testing and skin biopsy, final diagnosis and clinical impact. Results: A total of 52 patients, from 48 families were seen for 56 appointments for between 1st January 2013 and 31st December 2018. 26 were male and 26 female with an age range of 13 months to 38 years (median age $7 \mathrm{yr} 6$ months). We present, using illustrative cases how the combined clinic has made or changed a patient's diagnosis and impacted management. Conclusion: This study describes the WA experience in running a combined dermatology-genetics clinic. We believe that this model has benefited patients and their relatives in providing individualized specialist care.

\section{Multimodal Imaging Reveals Novel Clinical Features in CRB1-associated Retinopathy: The Asymptomatic Fenestrated Slit Maculopathy}

Danial Roshandel ${ }^{1}$, Jennifer A. Thompson ${ }^{2}$, Rachael C. Heath Jeffery ${ }^{1}$, Enid Chelva ${ }^{2}$, Terri L. McLaren ${ }^{1,2}$, Tina M. Lamey ${ }^{1,2}$, John N. De Roach ${ }^{1,2}$, Shane R. Durkin ${ }^{3,4}$ and Fred K. Chen ${ }^{1,5,6}$

${ }^{1}$ Centre for Ophthalmology and Visual Science (incorporating Lions Eye Institute), The University of Western Australia, Perth, Australia, ${ }^{2}$ Australian Inherited Retinal Disease Registry and DNA Bank, Department of Medical Technology and Physics, Sir Charles Gairdner Hospital, Perth, Australia, ${ }^{3}$ Discipline of Ophthalmology and Visual Science, The University of Adelaide, Adelaide, Australia, ${ }^{4}$ Department of Ophthalmology, The Royal Adelaide and Queen Elizabeth Hospital, Adelaide, Australia, ${ }^{5}$ Department of Ophthalmology, Royal Perth Hospital, Perth, Australia and ${ }^{6}$ Department of Ophthalmology, Perth Children's Hospital, Perth, Australia

Background: Biallelic CRB1 mutations can present as Leber congenital amaurosis (LCA), retinitis pigmentosa (RP) or cystic maculopathy. Aim: This study describes the novel multimodal imaging features of and expands on the varied CRB1-associated retinal disease phenotypes including the new entity of asymptomatic fenestrated slit maculopathy (AFSM). Methods: Twelve patients from nine families with CRB1-associated retinopathy were recruited. All patients were examined clinically and underwent ultra-widefield (UWF) colour fundus photography, fundus autofluorescence (FAF), spectral-domain optical coherence tomography (SDOCT), microperimetry and adaptive optics (AO) imaging to ascertain phenotype. Genotype and phase was determined using the Vision Panel and targeted Sanger sequencing, respectively (MVL, Oregon, US). Pathogenicity was assessed based on ACMG criteria. Results: Two broad phenotypes were observed on UWF FAF imaging: a diffuse retinopathy (1 LCA and $5 \mathrm{RP}$ ) and a localized maculopathy (4 symptomatic and 2 asymptomatic). FAF demonstrated the pathognomonic preserved para-arteriolar retinal pigment epithelium (PPRPE) sign in all RP patients. A unique macular volume profile on OCT scan was noted in patients with RP or symptomatic maculopathy. Microperimetry documented preserved foveal function in 6 patients. Two asymptomatic siblings with CRB1 variants c. [2843G $>$ A];[498_506del] had AFSM, characterized by localized outer retinal disruption and parafoveal cone abnormalities on AO imaging despite normal visual acuity and foveal sensitivity.
Conclusions: UWF imaging modality is useful in detecting the PPRPE sign which assists clinical genetics evaluation. Microperimetry and macular volume profiles may be useful endpoints for monitoring CRB1-associated retinal disease. AFSM is a new phenotype associated with the in-frame deletion c.498_506del variant.

\section{Are They Incidental, Secondary or Additional Findings? A Survey of Opinions and Practices Across Australian Genetic Testing Laboratories}

Emma Tudini ${ }^{1,2}$, Matilda Haas ${ }^{2,3}$, Tessa Mattiske ${ }^{2,3}$, Amanda Spurdle ${ }^{1}$ and on behalf of Australian Genomics

${ }^{1}$ QIMR Berghofer Medical Research Institute, Brisbane, Australia, ${ }^{2}$ Australian Genomics Health Alliance, Australia and ${ }^{3}$ Murdoch Children's Research Institute, Melbourne, Australia

As the use of large panels, whole exome and whole genome sequencing increases, so arises the potential to uncover clinically relevant genetic variants unrelated to the genetic test request - variously termed 'incidental', 'secondary' or 'additional' findings. In 2017, we conducted a survey of Australian clinically accredited genetic testing laboratories to evaluate practices around annotation, storage, curation and reporting of such findings. Laboratories were re-surveyed in April 2020 to seek updated opinions. Responses were analyzed for 17 laboratories performing single gene to whole genome sequencing. Findings include: (1) Consistent with the previous survey, there is no Australian consensus on a term. Further, 8/17 laboratories indicated that they use multiple terms: $2 / 8$ alternated depending on the context of the finding, and $3 / 8$ used two or more terms interchangeably. (2) Services performing panel testing were more likely to indicate that they would report-back findings outside of testing indication in relation to carrier status, pharmacogenetic markers, and adult-onset disorders in adult and pediatric cases.(3) $13 / 17$ laboratories are notified via the consent/test request form about whether a patient has consented to return of these findings. (4) $15 / 17$ laboratories store findings in laboratory databases, even if not reported. (5) All 9 WES/WGS services do not routinely analyze a list of medically actionable genes outside of the patient's indication for testing (e.g. ACMG 59). Thematic analysis of written policies from seven laboratories indicated little consistency in their content. These findings provide rationale to explore development of consistent policies around report-back of incidental/secondary/additional findings in Australia.

\section{Cryptic Splice Site Mutations in the ANO10 Gene Cause Autosomal Recessive Cerebellar Ataxia}

Sean Massey ${ }^{1}$, Yiran Guo ${ }^{2}$, Lisa G. Riley ${ }^{3,4}$, Nicole J. Van Bergen ${ }^{1,10}$, Sandra

A. Sandaradura ${ }^{4,5}$, Elizabeth McCusker ${ }^{6}$, Michel Tchan ${ }^{7}$, Mark Davis ${ }^{8}$, Daphne Smits ${ }^{9}$, Grazia M. S. Mancini ${ }^{9}$, Hakon Harkonarson ${ }^{2}$, Sandra Cooper ${ }^{4}$ and

John Christodoulou ${ }^{1,10}$

${ }^{1}$ Brain and Mitochondrial Research, Murdoch Children's Research Institute, Melbourne, Australia, ${ }^{2}$ Center for Applied Genomics, Children's Hospital of Philadelphia, Pennsylvania, USA, ${ }^{3}$ Rare Diseases Functional Genomics, Kids Research, The Children's Hospital at Westmead and The Children's Medical Research Institute, Sydney, Australia, ${ }^{4}$ Discipline of Child and Adolescent Health, University of Sydney, Sydney, Australia, ${ }^{5}$ Sydney Children's Hospitals Network, Sydney University, Sydney, Australia, ${ }^{6}$ Department of Neurology, Westmead Hospital, Sydney University, Sydney, Australia, ${ }^{7}$ Department of genetic Medicine, Children's Hospital at Westmead, Sydney University, Sydney, Australia, ${ }^{8}$ Diagnostics Genomics Pathwest Laboratory Medicine, Perth, Australia, ${ }^{9}$ Department of Clinical Genetics, Erasmus University Medical Center, Rotterdam, the Netherlands and ${ }^{10}$ Department of Paediatrics, Melbourne University, Melbourne, Australia 
Background: Spinocerebellar ataxia, autosomal recessive 10 (SCAR10) is a distinct classification of cerebellar ataxia caused by biallelic variants in the $A N O 10$ gene. Despite its pathophysiological relevance, $A N O 10$ is poorly characterized, but thought to be an ER scramblase aiding the regulation of $\mathrm{Ca} 2+$ homeostasis. Determining the clinical and functional significance of novel variants in $A N O 10$ is essential for accurate genetic diagnosis and therapeutics. Aims: Our aim was to assess the clinical significance of novel disease variants in the ANO10 gene. Methods: Patients diagnosed with spinocerebellar ataxia underwent massively parallel sequencing. ANO10 (NM_018075.3) variants were identified and validated in patient fibroblast by western blot and cDNA studies. Results: Two unrelated patients were identified with a homozygous intronic variant (c.1163$9 \mathrm{~A}>\mathrm{G}$ ) producing a leaky cryptic splice site which produces two alternative transcription products. RT-PCR showed one transcript (p.(Glu388Valfs*3)) has an inclusion of 8 base pairs of intronic sequence, and the other (p.(Glu388Val*69)) with complete skipping of exon 6. A third patient with atypical onset at 9 months old is potentially the youngest individual reported to-date with an expanded SCAR10 phenotype. The homozygous single nucleotide variant (c.1864A>G; p.(Met622Val)) within exon 11 leads to a new splice site, resulting in skipping of exon 12. Conclusions: We present two novel ANO10 variants to add to the growing list of ANO10 variants associated with a SCAR10 phenotype, which potentially includes the youngest individual reported to date. Together, these findings highlight the importance of ANO10 in human disease, providing invaluable information on the clinical spectrum of ANO10-related SCAR10 pathology.

\section{A Rare Case of Isolated Fetal Pericardial Effusion, Congenital Nephrotic Syndrome and Adrenal Calcification}

Kaitlin McGinnis ${ }^{1}$ and Fiona McKenzie ${ }^{1}$

${ }^{1}$ Genetic Services WA, Perth, Australia

Isolated fetal pericardial effusion is an uncommon occurrence. Reported associations include Down syndrome, RASopathies, congenital heart disease and other causes of evolving hydrops fetalis. In this case, an otherwise healthy fetus had a pericardial effusion and underwent frequent fetal anatomy and wellbeing scans, with no additional hydropic or anatomical abnormalities detected. Karyotype, chromosomal microarray and RAS gene panel testing were inconclusive on amniocyte sample. Unexpected postnatal findings included hypogonadism, facial dysmorphism, mild ichthyosis and an episode of hypoglycemia with depressed cortisol response. Further investigation revealed a structurally normal heart with complete resolution of pericardial effusion by the 5 th day of life. Repeat ultrasound imaging of the abdomen showed progressive adrenal calcification and ACTH stimulation testing provoked no response. Massive proteinuria and electrolyte imbalance developed, consistent with congenital nephrotic syndrome. Renal function continued to decline and suitability for dialysis had been debated. The leading differential in this case is sphingosine-1-phosphate lyase 1 (SGPL-1) mutation and preliminary biochemical testing suggests deficiency in this pathway. Genetic testing of the SGPL-1 gene is ongoing at present. This unusual condition should be considered in the context of fetal pericardial effusion, recognizing that other phenotypic features may develop later in the neonatal period. Adrenal and renal function should be closely monitored.

\section{The Expanding Phenotypic Spectrum of Verheij Syndrome}

Andrew Paul Fennell ${ }^{1,2}$, Smitha Kumble $^{3}$, Colina McKeown ${ }^{4}$, Gemma Poke ${ }^{4}$, Zornitza Stark ${ }^{3,5}$, Chloe Alice Stutterd ${ }^{3}$, Tiona Yang Tan ${ }^{3,5}$, Alison Yeung ${ }^{3,5}$, Ella Jane Wilkins $^{3}$ and Matthew Frank Hunter ${ }^{1,2}$

${ }^{1}$ Monash Genetics, Monash Health, Melbourne, Australia, ${ }^{2}$ Department of Paediatrics, Monash University, Melbourne, Australia, ${ }^{3}$ Victorian Clinical Genetics Services, Murdoch Children's Research Institute, Melbourne, Australia, ${ }^{4}$ Genetic Health Service New Zealand, Wellington Hospital, Wellington, New Zealand and ${ }^{5}$ Department of Paediatrics, The University of Melbourne, Melbourne, Australia

Verheij syndrome is a rare craniofacial spliceosomopathy presenting with craniofacial dysmorphism, multiple congenital anomalies and variable neurodevelopmental delay. It is caused by SNVs in PUF60 or interstitial deletions of the chromosome 8q24.3 region. PUF60 encodes a splicing factor which forms part of the spliceosome. To date, 27 patients with Verheij syndrome due to disease-causing PUF60 SNVs have been detailed substantively in peer-reviewed publications. Although the depth of their phenotyping has varied greatly, they exhibit marked phenotypic heterogeneity. We report five additional unrelated patients, including the first described patients of Khmer and Indian ethnicities and the eldest patient to date, with five heterozygous PUF60 variants identified through exome sequencing, four previously unreported. These included three frameshift (p.(Pro385Leufs*12), p.(Gly268Alafs*18) and p.(Met287Ilefs*5)), one missense (p.(Arg146Cys)) and one inframe deletion variant (p.(Ala150_Phe152del)). All patients underwent deep phenotyping identifying variable dysmorphism, growth delay, neurodevelopmental delay, and multiple congenital anomalies, including several unique features. Our adult patient is the only individual reported not to have had either neurodevelopmental delay or intellectual disability. In combining these detailed phenotypic data with the previously reported patients we further refine the known frequencies of features associated with Verheij syndrome. These include neurodevelopmental delay/Intellectual disability (97\%), short stature (60\%), limb anomalies (66\%), axial skeletal anomalies (57\%), cardiac anomalies (61\%), brain malformation (57\%), hearing loss (48\%), colobomata (32\%) and other ocular anomalies (61\%). This case series, incorporating two patients from previously unreported ethnic backgrounds, further delineates the mutational spectrum and unpredictable pleiotropy of PUF60 pathogenic variants.

\section{Spondylocostal Dysostosis with Anal Atresia and Urogenital Anomalies in an Infant: First Case Entity of Casamassimae Mortone Nance Syndrome from Asia}

Prashant Kumar Verma ${ }^{1}$, Radhapyari Lourembam ${ }^{1}$, Swathi Chacham ${ }^{1}$, Vyas Rathaur ${ }^{1}$, Raksha Ranjan ${ }^{1}$, Nowneet Kumar Bhat ${ }^{1}$ and Poonam Sherwani ${ }^{2}$

${ }^{1}$ Department of Pediatrics, All India Institute of Medical Science, Rishikesh, India and ${ }^{2}$ Department of Radiodiagnosis, All India Institute of Medical Science, Rishikesh, India

Casamassimae Mortone Nance syndrome (CMNS) includes a heterogenous group of spondylocostal dysostoses along with anal atresia and genitourinary abnormalities. In 1981, Casamassima et al. first described the syndrome in a fetus, and since then only seven such cases have been reported so far in the literature. There is significant phenotypic variability within the individual cases published so far. Causative genes of this rare syndrome are not yet known. Here, we report on a 3month-old female infant born out of nonconsanguineous marriage with normal karyotype and spondylocostal dysostosis, and anal and 
genitourinary malformations suggesting of CMNS. The additional features of sacral agenesis and bilateral clubfoot have not been documented earlier in the literature. Our case is the eighth reported case of CMNS, expanding the phenotypic spectrum and giving new insight into the severity of expression of malformations in CMNS.

\section{CYTOGENETICS}

\section{The Importance of Further Investigation for 10;11 Rearrangements in Acute Monoblastic/Monocytic Leukemia}

Eleanor Chiu ${ }^{1}$, Joanne White ${ }^{1}$, Adrian Zordan ${ }^{1}$, Lee Harrison ${ }^{1}$, Nicole Hatzopoulos ${ }^{1}$ and Slavisa Ninkovic ${ }^{1,2,3}$

${ }^{1}$ Victorian Cancer Cytogenetics Service, Melbourne, Australia, ${ }^{2}$ Department of Haematology, St Vincent's Hospital, Melbourne, Australia and ${ }^{3}$ Department of Medicine, University of Melbourne, Melbourne, Australia

Presented is the case of a 63-year-old male with new acute monocytic leukemia. Conventional cytogenetics showed an apparently straightforward reciprocal 10;11 translocation with breakpoints at 10p?12 and 11q13. An investigation with fluorescence in situ hybridization (FISH) on metaphases with the KMT2A (11q23) break apart probe revealed the involvement of KMT2A on the derivative 10 chromosome. Multicolour band (M-Band) FISH, for chromosomes 10 and 11 , further demonstrated the complex nature of the $t(10 ; 11)$ and confirmed the breakpoint on 10 at p12, the location of MMLT10. Rearrangements leading to a 5'KMT2A-3'MLLT10 fusion gene are a well recognized entity in acute monoblastic/monocytic leukemia and invariably require a complex rearrangement to be formed. In order to confirm the presence of such, this case demonstrates the importance of further investigation in cases of acute monoblastic/ monocytic leukemia with abnormalities involving 10p and 11q.

\section{Application of a Magnetic Cell Sorting Method to Cultured Cells for Fish Analysis of CD138-positive Cells in Plasma Cell Myeloma}

Rebecca Bowen ${ }^{1}$, Bruce Mercer ${ }^{1}$, Kathleen Rayeroux ${ }^{1}$, Karen Dunn ${ }^{1}$ and Pooi Yoke Chin ${ }^{1}$

${ }^{1}$ Victorian Cancer Cytogenetics Service, Melbourne, Australia

The Victorian Cancer Cytogenetics Service receives around 800 myeloma samples per annum. With the introduction of an expanded FISH panel for myeloma patients, the continuation of cytoplasmic immunoglobulin (cIg) staining as the method of plasma cell (PC) identification would result in FISH analysis becoming very time intensive and thereby increase turn-around times. Magnetic sorting (MCS) of CD138-positive (CD138+) cells was proposed as a solution. Introducing CD138+ MCS to the laboratory, would potentially reduce FISH analysis time but added complexities to the laboratory's workflow: a recommended $24 \mathrm{hr}$ window for MCS from the time of sample collection, as well as a higher financial cost per patient - both required mitigation. Shin et al. (2012) showed that samples may be cultured for up to 96 hours before magnetic cell sorting is performed with results comparable to those of samples sorted directly. FISH analysis was performed comparing fixed cIg stained cell preparations and cultured CD138+ MCS preparations on 15 patients received between 28 May and 16 June 2020. We found a comparable abnormality rate, a very high percentage of abnormal cells in the cell sorted cohort and a significantly reduced scoring time on FISH slides prepared from the MCS suspensions. Culturing cells for between 24 and 96 hours has allowed for effective 'triage' of new and follow-up myeloma samples arriving in the laboratory. This has given the flexibility to only use the more expensive MCS method on patients with a suitable PC percentage in the bone marrow and with a final diagnosis of myeloma.

\section{Education, Ethics and Social Issues}

\section{Molecular Pathology Integration and Delivery in an Innovative Medical Laboratory Science Course}

Wouter Kalle ${ }^{1}$ and Noelia Roman ${ }^{1}$

${ }^{1}$ School of Biomedical Sciences, Charles Sturt Universty, Locked Bag 588, Bathurst, Australia

The Bachelor of Medical Science (Pathology) is an Australian Institute of Medical Scientists (AIMS) accredited degree that has been taught at Charles Sturt University since 1987 (with constant review and modifications). With the increasing use of genetic testing in pathology laboratories a 'Molecular Pathology' subject was added as a capstone during a course review in 2012. Over the years, student feedback on this subject focussed on students 'feeling unprepared for the complexity of the content', and 'struggling with the amount of information'. Students were positive about the extensive literature review assessment, including the ethics component, which they thought brought the subject together and improved their understanding of the complexity of molecular pathology. In 2018 a scheduled course review provided the opportunity to innovate course delivery and achieve better integration and scaffolding of Molecular Pathology throughout the course. This was achieved by composing a list of Blooms taxonomy and applying it to strict scaffolding of knowledge and constructive alignment in learning outcomes and assessment between foundation subjects, and the development of a connected molecular and histopathology cluster. Through the addition of a specialist textbook and linking connections between foundational knowledge focussed on human genetics and human biochemistry, students will be much more prepared for the complexities of Molecular Pathology. Linking Molecular Pathology to other pathology specializations will ensure our graduates will be much more thoroughly prepared for the increasing use of genetic technology application in the pathology laboratory to support clinicians in the diagnostication, prognostication and treatment of disease.

\section{Content Analysis of the Instances of Inclusivity of LGBTQI+ People in the Language and Images of Australasian Genetic Services Websites}

Louise Cilento ${ }^{1}$, Chris Jacobs ${ }^{1}$, Sarah Long ${ }^{1}$ and Alison McEwen ${ }^{1}$

${ }^{1}$ Graduate School of Health, University of Technology Sydney, Sydney, Australia

Background: Lesbian, Gay, Bisexual, Transgender, Queer, Intersex and associated people (LGBTQI+) encounter verbal, behavioral, and environmental 'microaggressions' that subtly reinforce negative assumptions, stereotypes, and stigmas and communicate hostility and derogation for their identities. Microaggressions can contribute to disparate health care access and experience. In contrast, visibility, inclusion, and recognition of gender identities and relationships contribute to equitable health care. We aimed to examine the inclusivity of Australasian genetic services websites. Methods: We conducted 
content analysis of websites identified through the Human Genetics Society of Australasia 'Find a Genetic Counselor' directory and subsequent search strategy that mimicked potential clients' actions. We analyzed website content using a coding framework of microaggressions adapted from the literature and classified content as instances of inclusivity or non-nclusivity. Results: We analyzed 63 websites representing 78 public and private genetic services. Majority of websites $(61.9 \%, n=39)$ displayed noninclusive instances while $30.2 \%$ of websites $(n=19)$ displayed inclusive statements. Noninclusive instances outnumbered inclusive instances 13 to 1 . Instances occurred over two of the nine microaggression categories, Endorsement of Heteronormative or Gender Normative Culture and Environmental Microaggressions. Discussion: Inclusivity and non-inclusivity were not mutually exclusive, instead every website that displayed inclusivity also displayed at least one instance of noninclusivity. Our findings suggest that Australasian genetic services websites should be revised and informed by the views of LGBTQI+ people to promote effective engagement and inclusivity.

\section{Visibility of Transgender and Gender Nonconforming People and Relationships in Healthcare and Genetic Counseling: A Scoping Review}

Lucas Mitchell $^{1}$, Chris Jacobs ${ }^{1}$ and Alison McEwen ${ }^{1}$

${ }^{1}$ Graduate School of Health, University of Technology Sydney, Sydney, Australia

Background: Healthcare services may be ill-equipped to assist people who are Transgender or Gender Non-conforming (TG/GNC), resulting in microaggressions (unconscious subtle discriminatory actions and communication) and delays in accessing healthcare, further contributing to health disparities. We aimed to identify and map existing literature regarding the visibility of TG/GNC people and their relationships in healthcare to inform genetic counseling research. Method: We conducted a search of published and grey literature from five databases. Titles, abstracts and articles were independently screened by two reviewers until a good rate of inter-rater reliability was reached using Cohen's Kappa statistic. We conducted a narrative synthesis and followed the PRISMA reporting guidelines. Results: We included 18 articles of which three were related to genetic counseling. We mapped six themes. Four themes were about the experiences of TG/GNC people: (1) experiencing stigma and discrimination through multiple microaggressions, (2) researching clinics by seeking recommendations via social networks, the internet and befriending healthcare staff, (3) adopting an educator role to combat providers' lack of knowledge and openness and (4) remaining vigilant of providers' behavior after disclosing TG/GNC identity. Two further themes were about the lack of inclusivity in the clinical environment: (5) little representation of TG/GNC people and (6) minimal acknowledgement and normalization of relationships and support networks. Conclusion: Despite raised awareness of TG/GNC issues over the last decade, visibility is lacking in the healthcare environment generally and in genetic counseling specifically. Further research is needed to develop appropriate resources, visibly welcoming clinical spaces, and training for clinicians.

\section{Genetic Literacy In Multicultural Queensland: Design, Delivery and Evaluation of Medical Interpreter Training in Genetic Terminology}

Lindsay Fowles ${ }^{1}$, Miranda Vidgen ${ }^{2}$, Satrio Nindyo Istiko ${ }^{3}$, Katrina Cutler ${ }^{3}$, Kate Sullivan ${ }^{3}$, Erin Evans ${ }^{3}$, Stephanie Best ${ }^{4,5}$, Keri Finlay ${ }^{5,6}$, Priya Ramarao-Milne ${ }^{2}$ and Nicola Waddell
${ }^{1}$ Genetic Health Queensland, Brisbane, Australia, ${ }^{2}$ QIMR Berghofer Medical Research Institute, Brsibane, Australia, ${ }^{3}$ Queensland Genomics, Brisbane, Australia, ${ }^{4}$ Macquarie University, Sydney, Australia, ${ }^{5}$ Australian Genomics, Melbourne, Australia and ${ }^{6}$ Genetic Support Network Victoria, Melbourne, Australia

Background: Medical interpreters provide a vital service within the health system for patients with limited or no English language skills. Medical terms related to genetics are not universally part of medical interpreters' specialist training in Australia. Language skills in specialist areas tend to come from work-based practise or post-certification professional development. Aim: To develop and evaluate an online training program for Medical Interpreters on genetic concepts. Methods: In July/August 2020, three 2-hour interactive, online training sessions on genetic concepts were held in English with a mixed language cohort of medical interpreters. The training program included presentations on basic and applied genetics, two interactive activities using group reflection, and case studies. Evaluation was undertaken with pre/post-event surveys that explored changes in participant knowledge, self-efficacy, attitudes and behavior, and session experience. Results: 118 Medical Interpreters attended the training sessions with 33 and 43 participants completing the pre- and post-surveys, respectively. Training sessions improved participant knowledge $(p<.01)$. While data trends indicated improved self-efficacy and attitudes, these were not statistically significant observations. The majority of participants agreed that training was useful (93\%) and relevant $(79 \%)$ to their work, and more participants agreed that the case study activity (86\%) benefitted learning more than the reflective activity (58\%). Discussion: This online training program was well received by Medical Interpreters, and demonstrated improved knowledge in genetic concepts. Further work is needed to determine if this format of training for Medical Interpreters results in changes to practise behavior or improved patient outcomes.

\section{The National Strategic Action Plan for Rare Diseases: A Collaborative Multi-stakeholder Approach to Effective Rare Disease Policy Reform}

Nicole Millis ${ }^{1}$

${ }^{1}$ Rare Voices Australia, Melbourne, Australia

Rare Voices Australia (RVA) led the collaborative development of the National Strategic Action Plan for Rare Diseases (Action Plan) - the first coordinated effort to address rare diseases (RD) in Australia. The Action Plan addresses the many commonalities of RD within: awareness and education; care and support; and research and data. It outlines a comprehensive, collaborative and evidencebased approach built on 3 principles: person-centred; equity of access; and, sustainable systems and workforce. Aim: A review of strengths and learnings from the collaborative multi-stakeholder development of the Action Plan and how that has impacted RD policy reform. Methods: (1) Case study of national policy development which used multiple methods of qualitative data collection/analysis through a national process of stakeholder consultation. (2) Case studies of how this policy development has impacted RD policy reform. Results: National stakeholder consultation resulted in a significant amount of information from various perspectives. While there was diversity, there were many commonalities among RD stakeholders. The commonalities identified through the stakeholder consultations guided the Action Plan. A review of this policy development process highlights the importance of collaboration and multi-stakeholder engagement — both similarly important for policy reform 
that has begun in response to the Action Plan. Conclusion: The Action Plan is an effective case study of collaborative and multistakeholder policy development. Stakeholder consultation was central to its development and continues to be important through implementation. Case studies demonstrate the opportunity for the RD sector to drive implementation of the Action Plan and to bring positive systemic change.

\section{Impact of Rapid Exome Sequencing of Critically Ill Newborns on the Parent-Child Relationship}

Hilary Bowman-Smart ${ }^{1,2}$, Danya Vears ${ }^{1,4}$, Zornitza Stark ${ }^{1,2,3,5}$ and Christopher Gyngell ${ }^{1,2}$

${ }^{1}$ Murdoch Children's Research Institute, Melbourne, Australia, ${ }^{2}$ Department of Paediatrics, University of Melbourne, Melbourne, Australia, ${ }^{3}$ Victorian Clinical Genetics Services, Melbourne, Australia, ${ }^{4}$ Melbourne Law School, University of Melbourne, Melbourne, Australia and ${ }^{5}$ Australian Genomics Health Alliance, Melbourne, Australia

Rapid exome sequencing (rES) of critically ill infants provides diagnoses much more quickly than traditional genetic testing, impacting treatment decisions and clinical management. Yet rES raises complex ethical issues, such as how a diagnosis in such early stages of life can impact the parent-child relationship. Between 2018 and 2019, 108 critically ill infants underwent rES as part of a multisite Australian feasibility study, with $51 \%$ receiving a diagnosis. We explored the attitudes and experiences of 61 parents involved in the study using a mixed methods survey. Quantitative data was analyzed using the t-test and Fisher's exact test. Qualitative data was analyzed using content analysis. Receiving a diagnosis or a possible associated variant was correlated with parents having altered thinking about their child $(p=.018)$. Using the PedsQL 2.0 Family Impact Module, we found that the sample had a mean family functioning score of 50.4 out of 100. Parents expressed that having testing for their child had several benefits, such as allowing them to feel informed, accept the diagnosis, and prepare for challenges ahead. Parents mentioned that the information helped them make decisions about the most appropriate care for their child. However, parents also reported grief and fear for their child's future, with some finding the result difficult to accept. As the potential for implementing rES for infants in the Australian healthcare system increases, these results can help inform how genetic health professionals can best support parents and families, both when the test is offered and after result delivery.

\section{Genetic Counseling Education in Australasia: A Response to COVID}

Jan Hodgson ${ }^{1}$, Helen Mountain ${ }^{2}$, Melody Menezes ${ }^{1}$, Chris Jacobs ${ }^{2}$ and Alison McEwen ${ }^{2}$

${ }^{1}$ Department of Paediatrics, University of Melbourne, Melbourne, Australia and

${ }^{2}$ Graduate School of Health, University of Technology Sydney, Sydney, Australia

Background: Genetic counselor education in Australasia is currently provided by two universities. The University of Melbourne has a well-established Masters program delivered on campus, with students living in Melbourne for most of their studies. The Masters program at University of Technology Sydney is offered primarily online, with students completing the program from their home state/country. COVID19 impacted allied health education globally, forcing development of innovative solutions to teach and assess clinical skills. UTS and UoM have worked together, with the HGSA Accreditation Committee, to ensure that graduating genetic counselors receive sufficient, quality, practical experiences to become competent and fit to practice. Methods: Frequent and on-going communication between the programs and the HGSA Accreditation Committee to trial and embed creative solutions to a complex problem. Results: We designed a range of authentic initiatives to develop quality clinical skills in students during a year in which attendance at clinical placement sites was restricted by the pandemic including: (1) extended simulation activities; (2) virtual clinical placement/virtual clinics; (3) role plays with practicing genetic counselors; (4) role plays between students from both programs. Conclusion: COVID-19 challenged educators to respond in creative and meaningful ways to ensure graduates are confident, competent and fit for practice. Emerging evidence from students, clinical placement supervisors and employers suggests these initiatives are valued by students and effectively prepare them for the workplace. Participating in role plays provided students with opportunities for connection, insight and reflection while helping build the capacity of practicing Australasian genetic counselors in student education.

\section{Genomics Education for Medical Specialists - A Blended Learning Approach}

Fran Maher ${ }^{1,2,3}$, Taryn Charles ${ }^{1,2,3,5}$, Elly Lynch ${ }^{1,2,5}$, Melissa Martyn ${ }^{1,2,3}$, Callum McEwan ${ }^{1,3}$, Amy Nisselle ${ }^{1,2,3,4}$ and Clara Gaff ${ }^{1,2,3}$

${ }^{1}$ Melbourne Genomics Health Alliance, Melbourne, Australia, ${ }^{2}$ Murdoch Children's Research Institute, Melbourne, Australia, ${ }^{3}$ The University of Melbourne, Melbourne, Australia, ${ }^{4}$ Australian Genomic Health Alliance, Melbourne, Australia and ${ }^{5}$ Victorian Clinical Genetics Services, Melbourne, Australia

Background: Genomics has a potential role in many areas of healthcare, but many medical specialists lack specific knowledge, skills and confidence to order and interpret genomic tests. Melbourne Genomics' workforce development strategy includes a blended learning course in clinical genomics for these specialists. Methods: We developed four self-directed, interactive online modules, e-book and infographic that introduced foundational genetics content and clinically-relevant concepts, processes and skills for germline and somatic genomics. Workshops (pediatric, adult and somatic/cancer) then focussed on clinical cases presented by clinicians with genomics expertise and small group discussions. Participants completed evaluation surveys at baseline, post-online modules and post-workshops. Results: 85 clinicians registered for the course (71\% Consultants/ Fellows, 23\% Registrars/Trainees; 16 specialties; 5-20+y experience). 61 registrants accessed online modules (29 completed all modules) and 71 attended workshops (32 pediatric; 23 adult; 27 cancer). $34 \%$ had previously ordered an exome/genome test. Self-rated and objective measures of knowledge, skills and confidence in genomic processes and interpreting test reports improved incrementally; for example, proportions of self-rated 'good-excellent' ability to interpret a test report more than doubled (36\% baseline, 68\% post-online modules, $88 \%$ post-workshops). Participants rated online modules helpful for learning more foundational and pragmatic concepts, and facilitated workshops more helpful for learning about clinical aspects such as identifying the right test for the right patient, interpreting reports and genetic counseling, yet the major gains in knowledge and confidence were seen after online modules. Conclusion: Blended learning offers a flexible and adaptable approach to clinical genomics education for medical specialists.

\section{Adapting to the Challenges of the Global Pandemic on Genetic Counselor Education: Evaluating Students' Satisfaction with Virtual Clinical Experiences}

Chris Jacobs ${ }^{1}$ and Alison McEwen ${ }^{1}$

${ }^{1}$ University of Technology Sydney, Sydney, Australia 
Background: Travel restrictions, physical distancing and limits to clinical placements due to the global pandemic have raised challenges for genetic counseling education. In response, we created authentic virtual experiences mimicking clinical practice: virtual clinical placements (VCP), including intake calls, consultations, teamwork and time management, and genetic counseling via virtual simulation (VS) with standardized clients. Aim: To evaluate students' satisfaction with learning from virtual clinical experiences. Methods: Virtual clinical experiences conducted since March 2020 involve online pre-brief, simulation and debrief. We anonymously evaluated student satisfaction with the experience using a validated satisfaction with simulation scale and free text comments. We analyzed the combined responses from one first year VCP and one second year VS using descriptive statistics and content analysis. Evaluation of a further VCP and two VS are underway. Results: To date, 36 students $(75 \%)$ completed the survey for two virtual clinical experiences. For $94.3 \%$ of students $(n=33)$ the experience created a sense of reality, $97 \%(n=35)$ were able to reflect on their clinical ability and recognize their clinical strengths and weaknesses, and $100 \%(n=36)$ considered the experience to be valuable for clinical learning. Comments indicated the VCP increased first year students' confidence about clinical placement and the VS prepared second year students for telehealth. Conclusion: Preliminary findings suggest that participation in authentic virtual clinical experiences enhances students' learning and prepares them for practice. Analysis of three further datasets will provide evidence from which to determine whether virtual clinical experiences should be included in genetic counselor education beyond the pandemic.

\section{'I Would Like to Think it's not Biased': How Community- Based Healthcare Practitioners in Australia are Learning about Consumer Genomics}

Chriselle Hickerton ${ }^{1,2}$, Bronwyn Terrill ${ }^{1,3,4}$, Amy Nisselle ${ }^{1,2,5}$, Belinda McClaren ${ }^{1,2,5}$ Debra Graves ${ }^{1,6}$, Kate Dunlop ${ }^{1,7}$, Marie Cusack ${ }^{1,8}$, Clara Gaff ${ }^{1,2,5}$ and Sylvia Metcalfe ${ }^{1,2,5}$

${ }^{1}$ Australian Genomics Health Alliance, Melbourne, Australia, ${ }^{2}$ Murdoch Children's Research Institute, Melbourne, Australia, ${ }^{3}$ Kinghorn Centre for Clinical Genomics, Garvan Institute of Medical Research, Sydney, Australia, ${ }^{4}$ UNSW Sydney, St Vincent's Clinical School, Sydney, Australia, ${ }^{5}$ The University of Melbourne, Melbourne, Australia, ${ }^{6}$ Royal College of Pathologists

Australasia, Sydney, Australia, ${ }^{7}$ Sydney School of Public Health, Faculty of Medicine and Health, University of Sydney, Sydney, Australia and ${ }^{8}$ Centre for Genetics Education, NSW Health, Sydney, Australia

Background: Interpretation of consumer genomic tests (CGT) is provided by some practitioners outside of clinical genetics settings despite questionable clinical validity and utility of these tests. Little is known about current CGT practice or education/training received by these practitioners. Aim: To explore education/training available to community-based practitioners relating to commercially available nutrigenomic and pharmacogenomic tests. Methods: Medical practitioners with an interest in integrative medicine (mostly GPs), private genetic specialists, pharmacists, dieticians, naturopaths, nutritionists, educators/trainers (online courses, mentors, tertiary, Colleges) and commercial CGT company representatives were recruited via purposive, convenience and snowball sampling. In-depth semi-structured interviews were recorded, transcribed and inductively co-coded. Categories were identified using a constant comparative approach. Results: Overall, 44 participants were interviewed: 25 practitioners; 13 educators; 6 CGT company representatives. Practitioner support for patients regarding CGT varied. Practitioner motivations for learning about CGT were largely a result of marketing to by a CGT company and/or patients/clients bringing test results to an appointment. Practitioner education was variable but mostly self-driven or delivered by CGT companies.
A perceived gap in education on CGT drove some educators to introduce it into courses. Views on training provided by CGT companies were mixed: 'better than nothing', 'a conflict of interest', need mix of training provided by companies and other avenues. Individuals from CGT companies believed they provide bias-free, evidence-based training. Overall, there was a perception Colleges and Societies should oversee training. Conclusion: Practitioners would benefit from clear guidelines and evaluation of education/training focussing on how CGT may benefit patients and test limitations.

\section{GENETIC COUNSELING}

\section{Multidisciplinary Renal Genetics Clinics: Family Perspectives and Preferences}

Ella Wilkins ${ }^{1,2}$, Catherine Quinlan ${ }^{3}$, Andrew Mallett ${ }^{4}$ and Zornitza Stark ${ }^{1,2}$

${ }^{1}$ Victorian Clinical Genetics Services, Melbourne, Australia, ${ }^{2}$ Murdoch Children's Research Institute, Melbourne, Australia, ${ }^{3}$ The Royal Children's Hospital, Melbourne, Australia and ${ }^{4}$ Royal Brisbane and Women's Hospital, Brisbane, Australia

Multidisciplinary renal genetics clinics (RGC) comprising nephrologists, clinical geneticists, and genetic counselors operate in 15 public hospitals across Australia with the goal of providing family-centred care and definitive molecular diagnoses to patients. However, little is known about family perspectives of multidisciplinary clinics or of undergoing genomic testing in this context. Patients having genomic testing were surveyed following initial RGC attendance and after results disclosure. We explored patient experiences of the clinic, perceived impact of the disease on the family, understanding of the test, and hopes and expectations relating to testing. Surveys included the Decision Regret, and Genetic Counseling Outcome scales. Response rates for survey one and survey two were $72 \%$ and $53 \%$ respectively. Most respondents preferred the multidisciplinary clinic model to seeing specialists in separate clinics $(n=123,70 \%)$. The majority of respondents understood a genomic test analyses many genes $(n=171,58 \%)$, causative variant(s) may $(n=157,91 \%)$ or may not be identified ( $n=126,73 \%)$, results may be of uncertain significance ( $n=126,74 \%$ ), and identified variant(s) may be passed down in the family $(n=157,91 \%)$. Following results disclosure, $81 \%$ $(n=71)$ of respondents correctly described their result. Respondents agreed they received enough information during preand post-test counseling $(n=160,94 \%$ and $n=81,91 \%)$ and had the opportunity to ask questions before consenting to testing $(n$ $=160,95 \%)$. Understanding patient and family experiences and opinions, and the short- and long-term impacts on families will guide the design and delivery of RGCs and associated genomic testing programs. A full author list is available online at www.kidgen.org.au.

\section{Telegenetics Literature Review}

Anita Gorrie ${ }^{1}$, Judy Gold ${ }^{2}$, Carolyn Cameron ${ }^{1}$, Molly Krause ${ }^{1,3}$ and Helen Kincaid ${ }^{1}$

${ }^{1}$ Monash Health, Melbourne, Australia, ${ }^{2}$ Independent Consultant, Melbourne, Australia and ${ }^{3}$ University of Melbourne, Melbourne, Australia

Telegenetics involves the use of technology (generally video conferencing) to remotely provide genetic services. A telegenetics platform is critical for those with limitations compromising their ability to attend in-person clinics. As the demand for remote genetics services increases, and amidst the COVID-19 pandemic with social distancing practices in place, we conducted a literature review to examine the benefits and limitations of telegenetics and explore the views 
of patients and health professionals utilizing telegenetics. Searches of the PubMed database identified 21 relevant primary studies for inclusion. Most studies found acceptability of telegenetics to be high among patients and health professionals and that telegenetics provided access to genetics services for underserved communities. The main benefits cited include cost-effectiveness and reduction in travel time for genetics services providing outreach clinics and patients who would otherwise travel long distances to access genetics. Patients appreciated the convenience of telegenetics including reduced wait times, although a minority of patients reported their psychosocial needs were not adequately met. Five studies compared outcomes between telegenetics and in-person services and findings suggested telegenetics patients had a similar level of knowledge and understanding of genetics and similar psychological outcomes following their appointments. Some studies reported challenges related to establishing rapport and reading and responding to verbal cues via telegenetics, while technical issues were not generally found to be a major limitation. Further research may be necessary to gather and examine data on how telegenetics outcomes compare to that of in-person genetic counseling and adapt services accordingly.

\section{Genetic Counseling and Client Mental Wellbeing: What is the Role of Australian-Based Genetic Counselors?}

Madeleine Harris ${ }^{1,2}$, Michelle de Silva ${ }^{1,2,3}$, Stephanie Best ${ }^{2,4,5}$ and Keri Finlay ${ }^{2,3,6}$

${ }^{1}$ The University of Melbourne, Melbourne, Australia, ${ }^{2}$ Murdoch Children's Research Institute, Melbourne, Australia, ${ }^{3}$ Victorian Clinical Genetics Services, Melbourne, Australia, ${ }^{4}$ Australian Institute of Health Innovation, Sydney, Australia, ${ }^{5}$ Australian Genomics Health Alliance, Melbourne, Australia and ${ }^{6}$ Genetic Support Network of Victoria, Melbourne, Australia

Background: The role of a genetic counselor is to assist individuals and their families to adapt to genetic information. However, a genetic counselor's role in client mental wellbeing is unclear. Mental wellbeing is an important component of overall health and may be affected during the adaptation to genetic information. It is essential to consider how mental wellbeing is viewed in genetic counseling practice. Aim: To investigate Australian-based genetic counselors' perceptions of client mental wellbeing, with the intention to inform future practice. Methods: Participants were recruited through the Human Genetics Society of Australasia and Australasian Society of Genetic Counsellors. Semi-structured in-depth interviews have been completed. Interviews were transcribed verbatim and analyzed thematically. Our analysis was framed by the capability, opportunity, motivation, behavior (COM-B) model, a framework used to understand an individual's behavior. Results: Twelve participants were interviewed. Analysis is underway, with early insights showing that GCs believe that mental wellbeing is a crucial part of their practice, and many state they would like more training and professional development in the area of mental wellbeing. Findings suggest that Capability plays a key role for common barriers, such as limited ongoing training, faced by genetic counselors when assisting clients' mental wellbeing, whereas Opportunity is an important factor enabling genetic counselors to support clients' mental wellbeing. Conclusion: This study provides an insight into how genetic counselors' perceive their role in client mental wellbeing, establishes strategies and resources that genetic counselors' use, and determines barriers and enablers in supporting the mental wellbeing of clients.

\section{Parents' Experience of Receiving Genomic Testing Results for their Child Through the Victorian Undiagnosed Disease Programme}

Jo Martinussen ${ }^{1}$, Michal Chalk ${ }^{1}$, Justine Elliott ${ }^{1,2}$ and Lyndon Gallacher ${ }^{1,2}$

${ }^{1}$ Department of Paediatrics, University of Melbourne, Melbourne, Australia and ${ }^{2}$ Victorian Clinical Genetics Services, Murdoch Children's Research Institute, Melbourne, Australia

Background: The Victorian Undiagnosed Disease Programme (UDP-Vic) utilizes deep phenotyping, advanced genomic sequencing and functional studies to diagnose children with rare disease where previous clinical testing has been nondiagnostic. Of 167 families analyzed, 62 (37\%) have received a diagnosis or strong candidate for diagnosis. 21 of these children have variants in novel or emerging genes. The impact of genomic technologies on Australian families remains largely unknown. Aim: To explore the experience of parents receiving genomic test results for their child through the UDP-Vic. Methods and recruitment: Semistructured interviews ranging in length from 25 to 105 minutes were conducted with 21 parents of children in the programme. Ten participants were parents of children who received a diagnosis through the programme; 11 were parents of children who remain undiagnosed. Interviews were audio recorded and transcribed verbatim with thematic analysis conducted on the transcripts. Results: While the experiences of families were varied, five shared themes emerged from the data. All parents described in detail searching for a diagnosis, the varied impact of receiving a result and experiencing mixed feelings, including relief and disappointment. Parents of both diagnosed and undiagnosed children were seeking connection with other families, and talked about hope as well as moving towards acceptance of their child. Conclusion: The findings demonstrate the shared experience of parents of children with rare disease both before and after a genomic test result. This has implications for genetic counselors and clinicians offering genomic sequencing and supporting families of children with rare disease.

\section{Impact of Direct-to-Consumer Genetic Testing on Australian Clinical Genetics Services}

Michael Millward ${ }^{1}$

${ }^{1}$ Tasmanian Clinical Genetics Service, Hobart, Australia

The increasing popularity of direct-to-consumer genetic testing (DTCGT) is thought to be creating a burden on clinical genetic services worldwide. However, no Australian studies have collected recent evidence regarding this impact. Anecdotal evidence and previous studies in this area have demonstrated that DTCGT-related referrals are being seen in public services. However, the impact of these referrals on services has not yet been quantified. The aim of this study was to assess the impact of DTCGT on Australian public genetic services. We contacted every publicly funded genetic service in Australia $(n=19)$ to be surveyed regarding DTCGT-related referrals over the past 10 years. This data was collected in RedCAP and analyzed using STATA. Eleven services reported over 100 DTCGTrelated referrals. Most (83\%) involved general practitioners seeking interpretation of DTCGT results. More than 30\% involved imputed risk estimates from third-party software tools. Services reported low validation rates for DTCGT results $(<10 \%)$, and variable procedures 
for managing DTCGT referrals, with most (8/11) lacking specific procedures. Our study helps quantify the impact of DTCGT on clinical genetics services and highlights the impact of imputed risk estimates.

\section{Uncovering the Psychosocial Impact of Undergoing Genetic Testing for Rare and Recently Discovered Genetic Epilepsies}

S. M. Nevin ${ }^{1,2}$, K. Barlow-Stewart ${ }^{1,5}$, C. E. Wakefield ${ }^{1,2}$, B. C. McGill ${ }^{1,2}$, F. LeMarne ${ }^{1,3}$, E. Beavis ${ }^{3}$, R. Macintosh ${ }^{4}$, R. C. Dale ${ }^{3}$, D. Gill ${ }^{3}$, K. Kothur ${ }^{3}$, R. Sachdev ${ }^{4}$, A. Bye ${ }^{1,3}$ and E. E. Palmer ${ }^{1,4}$

${ }^{1}$ School of Women's and Children's Health, UNSW Medicine, Sydney, Australia, ${ }^{2}$ Behavioural Sciences Unit, Kids Cancer Centre, Sydney Children's Hospital, Sydney, Australia, ${ }^{3}$ Department of Neurology, Sydney Children's Hospital Network, Sydney, Australia, ${ }^{4}$ Centre for Clinical Genetics, Sydney Children's Hospital, Australia and ${ }^{5}$ Discipline of Genomic Medicine, University of Sydney, Sydney, Australia

Genetic early-onset epilepsies represent a heterogeneous group of neurodevelopmental conditions associated with drug-resistant seizures and complex psychiatric and behavioral comorbidities. With over 400 genetic loci identified, genetic testing has become standard care and a genetic diagnosis is increasingly being reached in the acute care setting. In-depth interviews with parents $(N=26)$ of children with genetic epilepsies, recruited from the Sydney Children's Hospital Network, explored their information needs and the psychosocial impact of genetic testing. Interviews were transcribed, deidentified, line-by-line coded and thematically analyzed by three coders, using an inductive approach. Thematic saturation was reached. Parents relayed that supportive genetic counseling from the outset influenced their capacity to adapt to their child's genetic diagnosis. Beyond this, parents described the impact of chronic unpredictable seizures combined with limited prognostic information, as contributing factors to physical and emotional burnout, especially in acute care settings. A lack of societal recognition regarding the severity of their child's genetic epilepsy reportedly impeded access to appropriate supports. However, perceived support from healthcare professionals and peers was associated with greater capacity to reframe the genetic diagnosis and more positive adaptation to their child's condition. Our research has identified important clinical implications and provides compelling insight into parents' unmet psychosocial support needs. These include the critical need for early access to supportive timely genetic counseling, customized resources that empower parents to communicate their child's diagnosis and healthcare requirements, and access to specialized psychological supports. Findings can be translated to support clinicians in providing genetic care for high-need populations.

\section{'You don't know whats ahead': Genetic Counselors' Views of Genetic Testing, Newborn Screening and Preconception Carrier Screening for Adrenoleukodystrophy}

Natasha Luboya ${ }^{1}$, Beata Bajorek ${ }^{2}$ and Chris Jacobs ${ }^{1}$

${ }^{1}$ Graduate School of Health, Discipline of Genetic Counselling, University of Technology Sydney, Sydney, Australia and ${ }^{2}$ Graduate School of Health, Discipline of Pharmacy, University of Technology Sydney, Sydney, Australia

Background: Adrenoleukodystrophy (ALD) is a rare, X-linked recessive condition with variable onset and severity, presenting healthcare professionals with challenges around diagnosis and management.
This study aimed to explore the experiences of genetic counselors (GCs) in providing care to people with ALD and examining views towards genetic testing; specifically, newborn and preconception carrier screening. Methods: Australasian participants were recruited via snowball sampling and interviewed online using a semi-structured interview guide. Interviews were transcribed and thematically analyzed using manual-inductive coding and consensus agreement was reached by the research team. Results: Six GCs from various specialities participated (mean 16 years' clinical experience; having counselled between 1-20 persons with ALD). Three themes were related to GCs' views towards newborn and preconception carrier screening: (1) genetic screening provides options for families, (2) questioning the appropriateness of newborn screening, and (3) ethical and practical concerns around preconception carrier screening. Discussion/Conclusion: GCs' views towards ALD on newborn and preconception carrier screens encompassed their considerations of the benefits and disadvantages of each. Despite the potential benefits of newborn screening, lack of certainty associated with the variability of ALD led to questions about appropriateness. Although there were ethical and practical concerns around equity, psychosocial impacts and logistics of preconception carrier screening, screening does provide families with options. Despite the potential benefits of newborn and preconception carrier screening, there is caution among GCs about introducing these tests to families with increased risk of ALD. This warrants further investigation.

\section{Family Dyads and Decision Making in Genetic Health Conditions: A Systematic Literature Review}

Wai Ki Law, Ebony Richardson and Erin Turbitt

The Discipline of Genetic Counselling, The University of Technology Sydney, Sydney, Australia

Background: Through interactions in daily life, people influence each other's behaviors and mental processes, including decision-making about their health. Such 'dyadic' decision-making is increasingly being studied in healthcare research, with supportive evidence of spousal, health professional-patient and parent-child interactions and influences. Compared to other health information, genetic information can directly impact biologically related individuals. As such, it is important to understand dyadic decision-making about genetic health conditions among family members, though the extent to which this has been studied is unknown. Aim: To gather and summarize current evidence about dyadic decision-making in genetics, including identification of which decisions and under what conditions related dyads influence each other. Methods: A systematic literature review following PRISMA guidelines was conducted. Peerreviewed publications were included if they reported quantitative primary research about dyadic decision-making in genetics, published between August 1998 and August 2020 and written in English. Results: The search returned 3167 articles, of which 15 met the inclusion criteria. Most studies were in the context of cancer genetic testing $(n=7)$, or reproductive testing or screening $(n=5)$. Studies reported two broad categories of decisions with dyadic influence: undergoing screening or testing and learning risk information or sharing information with family. A variety of other factors were correlated between dyads such as attitudes, knowledge, behaviors and psychological wellbeing. Conclusion: Emerging evidence shows that dyads influence each other when making decisions relating to genetic testing and genetic information. Our findings emphasize the importance of considering both members of a dyad in the process of genetic counseling. 


\section{Additional Genomic Findings: Towards a Feasible, Effective Model of Care}

Melissa Martyn ${ }^{1,2,4}$, Elly Lynch ${ }^{1,2,3}$, Ling Lee ${ }^{1,2,4}$, Rona Weerasuriya ${ }^{1,2,4}$, Anaita KangaParabia $^{1,3}$, Rigan Tytherleigh ${ }^{1,2}$ and Clara Gaff ${ }^{1,2,3}$

${ }^{1}$ Murdoch Children's Research Institute, Melbourne, Australia, ${ }^{2}$ Melbourne Genomics Health Alliance, Melbourne, Australia, ${ }^{3}$ Victorian Clinical Genetics Services, Melbourne, Australia and ${ }^{4}$ Department of Paediatrics, University of Melbourne, Melbourne, Australia

Genomic sequencing for diagnostic purposes also enables the deliberate search for variants predictive of future health risk ('additional findings'). $\mathrm{AF}$ are not routinely offered in Australasia and evidence is lacking to guide development of a feasible, effective model of care. To specifically examine AF decision-making and preferences of Australians who have had genomic testing, we offered AF to 200 adults at a separate genetic counseling appointment after return of diagnostic results. Patients completed surveys after AF genetic counseling, after AF result return and 6 months following last contact. Transcripts of recorded consultations were analyzed to develop themes using a data-driven inductive method, while a theory-informed deductive method identified techniques applied to support patient decision-making processes. Kappa scores of independently coded transcripts indicated strong interrater reliability. Eighty-three $(83 / 200,42 \%)$ patients attended face-to-face pretest counseling with $98 \%$ accepting AF. Patients felt supported in decision-making: 95\% agreed the level of information received was 'about right' and (94\%) reported counseling helpful. Nonetheless, 34\% of participants mistakenly believed the test was for all known genetic conditions or would provide information about their primary condition. All 49 respondents to the post-result survey correctly recalled their AF result but confusion about its implications was evident. Transcript analysis revealed potential modifiable reasons for misconceptions, including genetic counselor assumptions and nonmodifiable reasons including impaired cognitive state. There was evidence of unmet support needs relating to diagnostic results. Provision of a feasible, effective AF service requires evidence-based strategies that address common AF misconceptions and are achievable in public health systems.

\section{The Morbidities Associated With ARID1B, ANKRD11, KMT2A, $D D X 3 X$ and $A D N P$-Related Conditions: A Systematic Review}

Cassie (Christina) Greer ${ }^{1}$, Christina Cassie Greer $^{1}$, Melanie Leffler ${ }^{2}$, Elizabeth Emma Palmer ${ }^{3}$ and Erin Turbitt ${ }^{1}$

${ }^{1}$ The Discipline of Genetic Counselling, University of Technology Sydney, Sydney, Australia, ${ }^{2}$ NSW Genetics of Learning Disability Service, Newcastle, Australia and 'School of Women's and Children's Health, UNSW Medicine, University of New South Wales, Sydney, Australia

Background: Adults with intellectual disability (ID) have reduced life expectancy and higher incidence of preventable deaths compared to the general population. Little is known about morbidities experienced by adults with a diagnosis of recently described single-gene causes of ID. Aim: To evaluate what is currently known about the medical and psychiatric morbidities associated with adulthood for the five commonest single-gene causes of ID diagnosed in the genomic sequencing era (ARID1B, ANKRD11, KMT2A, DDX3X and $A D N P$-related conditions). A secondary aim was to gather information needs of those affected and management recommendations. Methods: We conducted a systematic search of Medline, EMBASE, PsycINFO, CINAHL and Scopus. Titles, abstracts and articles were independently screened by two reviewers until good agreement was reached, then screened by CG. Quality assessments using the Joanna Briggs Institute Critical Appraisal tool and the QualSyst tool were performed. A narrative synthesis was conducted grouping human phenotype ontology terms, PRISMA reporting guidelines were followed. Results: Twenty-three articles were included. The medical morbidities associated with cardiovascular, neurological, endocrine and skeletal systems were frequently recorded for the four commonest conditions. Neuropsychiatric morbidities including autism spectrum, anxiety and attention deficit hyperactivity disorders were reported. There is a gap in the literature around ADNP-related conditions in adulthood, limited information on life expectancy, information needs and management guidelines for later adulthood for all conditions. Conclusion: Detailed natural history data for this group of conditions, particularly ADNP-related conditions, over the entire lifespan is required to inform accurate genetic counseling, improve health surveillance and potentially reduce premature mortality.

\section{Using Co-Design to Develop an Online Intervention for Families After Sudden Cardiac Death in the Young}

Laura Yeates ${ }^{1,3,4}$, Karen Gardner ${ }^{5}$, Judy Do ${ }^{1}$, Lieke van den Heuvel ${ }^{6,7}$, Gabrielle Fleming ${ }^{8}$, Christopher Semsarian ${ }^{2,3,4}$, Alison McEwen ${ }^{8}$, Leesa Adlard ${ }^{4}$ and Jodie Ingles ${ }^{1,3,4}$

${ }^{1}$ Cardio Genomics Program, Centenary Institute, Sydney, Australia, ${ }^{2}$ Agnes Ginges Centre for Molecular Cardiology, Centenary Institute, Sydney, Australia, ${ }^{3}$ Sydney Medical School, Faculty of Medicine and Health, The University of Sydney, Sydney, Australia, ${ }^{4}$ Department of Cardiology, Royal Prince Alfred Hospital, Sydney, Australia, ${ }^{5}$ Public Service Research Group, School of Business, UNSW Canberra, Canberra, Australia, ${ }^{6}$ Department of Clinical Genetics Amsterdam UMC, Amsterdam, the Netherlands, ${ }^{7}$ Department of Genetics, University Medical Centre Utrecht, Utrecht, the Netherlands and ${ }^{8}$ Graduate School of Health, University of Technology Sydney, Sydney, Australia

Sudden cardiac death (SCD) is a devastating complication of many genetic heart diseases. The psychological impact on surviving family members is significant and lifelong. Previous needs analysis found medical and psychological needs are of high importance but remain unmet in some cases. Co-design is an increasingly popular research method for the development of interventions and services that include users as members of the research team. Previous research has shown co-design leads to innovative ideas that better address user needs. Aim: To use co-design to develop an online support intervention for families after SCD. Methods: Semi-structured moderated online focus groups were held with key stakeholders, i.e. family members who had experienced young SCD, healthcare professionals and researchers. Guided discussions were used to co-design an online support intervention. Thematic analysis of discussions and iterative feedback on draft materials guided content development. Results: Four focus groups were held (average 11 participants per group). Stakeholder involvement facilitated development of highlevel ideas and priority issues. Key benefits included creative content and materials developed based on user preference for stories, narratives and information reflecting everyday experience of families navigating the legal and medical processes surrounding SCD, normalizing and supporting grief responses in the context of family relationships, and fostering hope. Conclusion: Co-design supported creative presentation, tailoring of information and development of psychosocial interventions to meet the needs of SCD families. Further work is needed to pilot and trial the intervention, prior to it becoming a key resource in the genetic counseling of these families. 


\section{Genetics and Genomics in Palliative Care: Exploring the Views and Experiences of Palliative Care Clinicians}

Stephanie White ${ }^{1,2}$, Chris Jacobs ${ }^{3}$ and Jane Phillips ${ }^{1}$

${ }^{1}$ Faculty of Health, University of Technology Sydney, Sydney, Australia, ${ }^{2}$ Department of Clinical Genetics, Royal North Shore Hospital, Sydney, Australia and ${ }^{3}$ Graduate School of Health, University of Technology Sydney, Sydney, Australia

Background: Palliative care (PC) represents the final opportunity to address genetics with a patient, and if required, collect a DNA sample that could provide life-changing information for their relatives. To support clinicians to address this potentially difficult topic with families, we must understand the factors helping and hindering the provision of genetic information to patients receiving PC. Aim: To explore the views and experiences of PC clinicians in addressing genetics with their patients and families. Methods: An interpretive descriptive qualitative study, utilizing semistructured interviews and focus groups with PC doctors and nurses. Data has been, and will continue to be, subjected to thematic analysis. Results: Early data from six interviews with PC doctors suggest that while, in most cases, other clinicians have raised genetics with patients before they enter PC, some have not been approached. Deciding to raise genetics with dying patients involves careful contemplation of the potential harms and benefits to the patient and relatives. PC doctors feel unequipped to discuss genetics in detail or organize testing and suggest incorporating genetics education into PC physician training. Discussion: Studies exploring the integration of genetics in Australasian PC are lacking. PC presents unique challenges to address genetics with patients, but initial and ongoing education may instil relevancy and capability while improving comfort. Although the medical aspects of genetics may not directly impact the patient's PC provision, increasing clinicians' abilities may help address psychological care and provide families with medically relevant information to guide screening or management and optimize their health.

\section{The Importance of Genetic Counseling to Prevent Patients' Doctor's Misinterpreting High Risk NIPT Screening Results}

Leslie Sheffield $^{1}$, Kathryn Visser ${ }^{1}$ and Edith Sheffield ${ }^{1}$

${ }^{1}$ MyDNA Life Australia Limited, South Yarra, Australia

Background: The advent of Non-Invasive Prenatal Testing (NIPT) has produced a number of providers that offer NIPT with varying counseling support. Aim and research question: To illustrate that there is still confusion among medical practitioners about the need and availability of genetic counseling and the positive predictive value of the test. The aim is to show that genetic counseling can lead to a more informed outcome. Hypothesis: Confusing information about the test often leads to false information being given. Methods: Telehealth counseling of patients referred for counseling about high risk NIPT results was provided. A case report reflective of many cases counselled is described. Results. A 25-year-old woman had positive results on NIPT for trisomy 18 (T18). The referring doctor informed her that the result showed T18 with 99\% accuracy. Termination was advised without discussion of the option of further diagnosis and genetic counseling, even though genetic counseling was freely available through the NIPT service. However, she was unhappy with this advice and eventually contacted the genetic counseling service. This counseling indicated that the chance the baby had T18 was $20 \%$, taking her age into account. A chorionic villus test confirmed T18. Discussion: The confusion of many practitioners about the high sensitivity and specificity compared with the positive predictive value often leads to gross overestimates of the predictive value given to patients. High sensitivity seems to limit the practitioners' understanding of the need for referral for genetic counseling. Genetic counseling can improve the information given to the patient.

\section{GENETIC EDUCATION}

\section{Co-Developing Psychological Resources for Families with Genetic Epilepsy}

S. M. Nevin ${ }^{1,2}$, C. E. Wakefield ${ }^{1,2}$, F. Le Marne ${ }^{3}$, E. Beavis ${ }^{3}$, R. Macintosh ${ }^{4}$, R. Sachdev ${ }^{1,4}$, Palmer ${ }^{1,4}$, A. Bye ${ }^{1,3}$ and K. Nunn ${ }^{5}$

${ }^{1}$ School of Women's and Children's Health, UNSW Medicine, Sydney, Australia, ${ }^{2}$ Behavioural Sciences Unit, Kids Cancer Centre, Sydney Children's Hospital, Sydney, Australia, ${ }^{3}$ Department of Neurology, Sydney Children's Hospital, Sydney, Australia, ${ }^{4}$ Centre for Clinical Genetics, Sydney Children's Hospital, Sydney Australia and ${ }^{5}$ Department of Psychological Medicine, Children's Hospital at Westmead, Sydney, Australia

Parents of children with genetic epilepsy have high rates of psychological morbidity and report a sparsity of accessible and acceptable psychological supports. Optimal methods of providing parents psychological support tailored to their child's complex disease trajectory remain unknown. We hypothesized that collaborating with parents to co-develop customized psychological resources will have a positive emotional impact on parents of a child with a rare genetic condition. The primary aim of this study was to co-design a customized and empirically informed digital resource. Second, we aimed to assess the acceptability and the perceived emotional impact of the resource. The content of the resources was iteratively codeveloped with parents, using in-depth interviews $(N=26)$ and focus group methodology $(N=7)$. Phase two (in progress) will assess the acceptability and the perceived emotional impact of the resources, using validated questionnaires. Parents reported that the content of the resources presented an accurate representation of their caregiving experiences and psychological support needs. Second, parents advised that the resources could be enhanced by; emphasizing the benefit that lies in embracing acceptance, reframing expectations to celebrate the joy of their child and striving towards empowerment in the face of ongoing challenges. Feedback was incorporated to inform further resource modifications. Six digital, thematically informed video resources were produced, with the titles; 'The uncertainty of a profoundly unwell child', 'Social isolation and accepting help', 'Relieving the struggle and embracing what is possible', 'Putting the pieces together and moving forward', 'The grieving process and freedom to embrace happiness', and 'Building hope and resilience'.

\section{GENOMICS}

\section{Proof-of-Principle: CRISPR-Cas9 Gene Editing for the Treatment of Immune Disorders}

Esther Elliott ${ }^{1}$

${ }^{1}$ Genomics Research Centre, IHBI, QUT, Brisbane, Australia

Background: Immune disorders such as immunodeficiency disorders often require lengthy treatments and are even fatal in some cases, presenting a significant clinical burden on Australian health care. 
Abstracts for the Human Genetics Society of Australasia Virtual Conference 24-25 November 2020

To pave the way for future interventions, further understanding of the utility of genome and transcriptome editing for the treatment of immune disorders will assist in establishing CRISPR-Cas gene therapy as a viable treatment option for patients with primary immunodeficiency disorder (PID). Research Problem: To date no established gene editing models are available to restore functional Moesin (MSN) expression in MSN-deficient patients. As such, long-term treatment to restore normal immune cell functioning to prevent disease in immune disorders is still lacking in current clinical practise. CRISPR-Cas9 editing may provide a solution to alleviate or cure specific immune disorders. Aims: (1) To undertake focused functional studies on MSN and TET2 implicated genes by designing and optimizing CRISPR-Cas9 gene editing approaches and analyses, including to specifically correct a mutation of an MSN, c.511C>T, p.Arg171Trp mutation, causally implicated in an X-linked PID. (2) To provide a proof-of-principle for the use of gene editing to restore MSN protein production in lymphocytes and rescue the lymphopaenic phenotype. Results and Discussion: CRISPR-Cas9 gene editing has successfully produced the desired MSN single base edit in a Raji B-cell line. Single-stranded DNA editing such as Prime Editing may potentially overcome low editing efficiency and the introduction of indels seen in some Cas9 applications. Further work is underway to establish single-stranded MSN editing to restore normal $\mathrm{T}$ and $\mathrm{B}$ cell functioning.

\section{Exome Sequencing for Infants Presenting With Isolated Congenital Hearing Loss: A Cost-Effectiveness Analysis}

Lilian Downie $^{1,2,3}$, David Amor ${ }^{1,3,4,5}$, Jane Halliday ${ }^{1,3}$, Sharon Lewis $^{1,3}$, Melissa Martyn ${ }^{1,3}$ and Ilias Goranitis ${ }^{1,3,6}$

${ }^{1}$ Murdoch Children's Research Institute, Melbourne, Australia, ${ }^{2}$ Royal Melbourne Hospital, Melbourne, Australia, ${ }^{3}$ Department of Paediatrics, University of Melbourne, Melbourne, Australia, ${ }^{4}$ Victorian Clinical Genetics Service, Melbourne, Australia, ${ }^{5}$ Royal Children's Hospital, Melbourne, Australia and ${ }^{6}$ Centre for Health Policy, University of Melbourne, Melbourne, Australia

Background: Exome sequencing is the gold standard investigation for hearing loss. Access to exome sequencing is limited due to the high cost of testing. Emerging evidence has demonstrated the clinical and personal utility of exome sequencing in early aetiological diagnosis of hearing loss. To ensure that the additional cost of performing exome sequencing is justified, a cost-effectiveness analysis is required. Aim: To assess the relative cost-effectiveness of exome sequencing for isolated congenital deafness compared with standard care. Methods: A decision tree was used to model the costs and outcomes associated with exome sequencing and standard care for infants presenting with isolated congenital deafness. Incremental cost-effectiveness and cost-benefit analyses were undertaken from the perspective of the Australian healthcare system using an 18-year time horizon. Results: Exome sequencing resulted in an incremental cost of AU $\$ 1000$ per child and an additional 30 diagnoses per 100 children tested. The incremental cost-effectiveness ratio was AU\$3333 per additional diagnosis. The mean societal willingness to pay for exome sequencing was estimated at AU $\$ 4600$ per child tested relative to standard care, resulting in a positive net benefit of AU\$3600. Deterministic and probabilistic sensitivity analyses confirmed the cost-effectiveness of exome sequencing. Discussion/Conclusion: Our findings demonstrate the cost-effectiveness of exome sequencing in congenital hearing loss, through increased diagnostic rate and consequent improved process of care by reducing or ceasing diagnostic investigation or facilitating targeted further investigation. We recommend equitable funding for exome sequencing in infants presenting with isolated congenital hearing loss.

\section{Single Molecule Analysis Enabled by DNA Co-Barcoding Technology}

Ou Wang ${ }^{1}$

${ }^{1} \mathrm{BGI}$-Shenzhen, Shenzhen, China

The advent of massively parallel sequencing has revolutionized life science research and brought forward a myriad of applications. Present mainstream methods for generating nucleic acid sequence information include: (1) highly-accurate and low-cost sequencingby-synthesis solution, albeit with relatively short read lengths (<500 bases); (2) single-molecule sequencing solution that enables sequence reads into the hundreds of kilobases in length, although less cost-effective and highly-erroneous. Specialized library preparation techniques such as DNA co-barcoding provide the best of both worlds as they enable long-read information up to hundreds of kilobases, using sequencing-by-synthesis systems. Nonetheless, these strategies present an intrinsic limitation in assembling the original molecule as it is virtually impossible to achieve $100 \%$ coverage of each DNA co-barcoded fragment. To overcome this limitation, we have developed a system that combines rolling circle amplification with the co-barcoding strategy. This approach increases the coverage per fragment above 1X, enabling a comprehensive single-molecule full-length analysis of fragments up to $20 \mathrm{~kb}$, and ultimately allows for highly-accurate single-molecule analyses of RNA, long amplicons, or selectively captured regions at the sequencing-by-synthesis cost and throughput.

\section{Detection and Reporting of Disease-Causing Repeat Expansions in a Clinical Whole Genome Sequencing Test}

Tanner Hagelstrom ${ }^{1}$, Aditi Chawla ${ }^{1}$, Egor Dolzhenko ${ }^{1}$, Vani Rajan ${ }^{1}$, Subramanian Ajay ${ }^{1}$, Michael Eberle ${ }^{1}$, David Bentley ${ }^{1}$, Denise Perry and Ryan Taft ${ }^{1}$

${ }^{1}$ Illumina, Inc., San Diego, United States

The CLIA-certified, CAP-accredited laboratory Illumina Clinical Services Laboratory (ICSL) performs clinical whole genome sequencing (cWGS) for patients with suspected genetic disease. Here we report on the validation and reporting of short tandem repeat (STR) expansion detection by cWGS. cWGS has the ability to test variant types across the genome, and a class-based variant validation strategy has been successfully applied to different variant types. We extended this practice to be able to detect STR expansion using the Expansion Hunter (EH) software package across nine loci representative of four different STR sequences (CGG, GAA, CAG and CTG). Analysis workflow involved a rubric that defined a given STR as 'expanded' if it showed sequencing read support beyond the pathogenic range, as well as a quality control step involving visual inspection of each variant call using sequence graph pileups. cWGS analysis of 168 patient previously tested samples with clinical diagnosis of Fragile X, myotonic dystrophy, spinocerebellar ataxia (SCA1, 2, 3, 6, and 7), spinal bulbar muscular atrophy, and Friedreich ataxia, revealed $97.9 \%$ sensitivity and $>99 \%$ specificity. The validation of STRs has allowed inclusion of twenty one STR loci as part of the cWGStest definition since November 2019. Of the 280 cases tested to date, 4 cases were identified with STR expansions, 2 myotonic dystrophy and 2 cases with Friedreich ataxia. 
These data demonstrate that WGS is able to detect STR expansions; further expanding the potential of WGS to serve as a comprehensive first-line test for patients with a suspected genetic disease.

\section{Comparing Reporting Outcomes for Proband-Only, DUO, and TRIO Cases in a Clinical Whole Genome Sequencing Test}

Amanda Buchanan ${ }^{1}$, Erin Thorpe ${ }^{1}$, John Belmont ${ }^{1}$, Carolyn Brown ${ }^{1}$,

Tanner Hagelstrom ${ }^{1}$, Denise Perry ${ }^{1}$ and Ryan Taft ${ }^{1}$

${ }^{1}$ lllumina, Inc., San Diego, United States

The Illumina Clinical Services Laboratory offers clinical whole genome sequencing for families with suspected genetic disease. Test outcomes are defined as: 'positive', 'negative', and 'see below'. Findings that may warrant a 'see below' report include the reporting of non-diagnostic variants or incidental findings. We assessed the impact of family structure on $630 \mathrm{cWGS}$ test outcomes between September 2017 and May 2020. Family structures included one sexto, five quint, 46 quad, 454 trio, 77 duo, and 47 proband-only cases. Among trios, $46 \%$ received 'positive' reports and $22 \%$ received 'see below' reports. In this group, de novo variants were identified in $62 \%$ with a 'positive' report and $18 \%$ with a 'see below' report. Among duos, $35 \%$ had 'positive' reports and $34 \%$ had 'see below' reports. Among probandonly cases, $36 \%$ had 'positive' reports and $34 \%$ had 'see below' reports. Among the proband-only cases with a 'see below' report, $88 \%$ included VUS variants in genes with phenotypic overlap with the proband. In this cohort, family structure significantly affected reporting outcome (Pearson's chi-squared test, $\chi^{2}=7.7553, d f=2$, $p=.02$ ). Trios were more likely to yield a 'positive' report, while proband-only cases were more likely to yield a 'see below' report $(\mathrm{OR}=1.97$, 95CI 1.21-3.19). Furthermore, de novo variants accounted for more than half of trios with a 'positive' report. When possible, submitting samples for the proband's family members to accompany the proband's sample should be considered to increase likelihood of diagnosis and reduce time in the diagnostic odyssey.

\section{Pediatric Mitochondrial OXPHOS Disorders: What Can a Retrospective 10-Year Birth Cohort Tell Us?}

Alison Compton ${ }^{1,2}$, Rocio Rius ${ }^{1,2}$, Hayley Mountford ${ }^{1,2,3}$, Sumudu Amarasekera ${ }^{1,2}$ Daniella Hock ${ }^{4}$, Nicole Lake ${ }^{1,2,5}$, AnneMarie Welch ${ }^{1}$, Ann Frazier ${ }^{1,2}$, Sarah Calvo ${ }^{6,7,8}$, Cas Simons $^{1,9}$, David Stroud ${ }^{4}$, Monkol Lek ${ }^{5}$, John Christodoulou ${ }^{1,2,10}$, Vamsi Mootha ${ }^{6,7,8}$ and David Thorburn ${ }^{1,2,10}$

${ }^{1}$ Murdoch Children's Research Institute, Melbourne, Australia, ${ }^{2}$ Department of Paediatrics, University of Melbourne, Melbourne, Australia, ${ }^{3}$ Department of Biological and Medical Sciences, Oxford Brookes University, Oxford, UK, ${ }^{4}$ Department of Biochemistry and Molecular Biology and Bio21 Molecular Science and Biotechnology Institute, University of Melbourne, Melbourne, Australia, ${ }^{5}$ Department of Genetics, Yale School of Medicine, New Haven, USA, ${ }^{6}$ Howard Hughes Medical Institute and Department of Molecular Biology, Massachusetts General Hospital, Boston, USA, ${ }^{7}$ Department of Systems Biology, Harvard Medical School, Boston, USA, ${ }^{8}$ Broad Institute, Cambridge, USA, ${ }^{9}$ Institute for Molecular Bioscience, The University of Queensland, Brisbane, Australia and ${ }^{10}$ Victorian Clinical Genetics Services, Murdoch Children's Research Institute, Royal Children's Hospital, Melbourne, Australia

Background: Mitochondrial OXPHOS disorders are clinically heterogeneous and comprise over 350 different genetic disorders. Aims: To obtain molecular diagnoses for all patients in a defined retrospective cohort of pediatric patients with OXPHOS disorders. Methods: Established retrospective cohort of 111 patients with OXPHOS disorders and onset of symptoms by 16 years, born between 1987-1996 in south-eastern Australia. The cohort represents all known children with a definite diagnosis of OXPHOS disease (according to Bernier criteria) in this region and time period. Molecular diagnoses were determined through numerous technologies as they have evolved; in the last 5 years these comprised whole exome or genome sequencing of all patients lacking a genetic diagnosis. Results: Currently, 97 patients (87\%) have a molecular diagnosis in 40 different genetic loci. Inheritance patterns were as expected with $63 \%$ autosomal recessive, $30 \%$ mtDNA-related, $5 \% \mathrm{X}$-linked and $2 \%$ de novo dominant. A molecular diagnosis was reached in all 16 patients with hepatic presentations with $81 \%$ caused by autosomal recessive POLG mutations. Cardiac patients have the lowest diagnostic rate at $67 \%$, however this has improved in the last year (from $<50 \%$ ) with the identification of de novo ATAD3 duplications. Fourteen patients still lack a genomic diagnosis and will undergo further 'omic' investigations (RNA-Seq and quantitative proteomics). Conclusion: Our study illustrates the increased ability to obtain molecular diagnoses in well-defined cohorts and the genetic complexity of mitochondrial disorders. It will allow us (with continued diagnoses) to better determine retrospective estimates of disease prevalence and natural history.

\section{Fatal Perinatal Mitochondrial Cardiac Failure Caused by Recurrent De Novo Duplications in the ATAD3 Locus}

Ann Frazier ${ }^{1,2}$, Alison G. Compton ${ }^{1,2}$, Yoshihito Kishita ${ }^{3}$, Daniella H. Hock ${ }^{4}$, AnneMarie E. Welch ${ }^{1}$, Sumudu S. C. Amarasekera ${ }^{1,2}$, Rocio Rius ${ }^{1,2}$, Luke E. Formosa ${ }^{5}$, Atsuko ImaiOkazaki $^{3,6}$, David Francis ${ }^{7}$, Min Wang ${ }^{1}$, Nicole J. Lake ${ }^{1,2,8}$, Simone Tregoning ${ }^{1,7}$, Belinda Chong ${ }^{7}$, Simone M. Rowley ${ }^{7}$, Jafar S. Jabbari ${ }^{9}$, Alexis Lucattini ${ }^{9}$, Kazuhiro R. Nitta ${ }^{3}$, Akira Ohtake ${ }^{10}$, Kei Murayama ${ }^{11}$, David J. Amor ${ }^{1,2}$, George McGillivray ${ }^{7}$, Flora Y. Wong ${ }^{12}$, Marjo S. van der Knaap ${ }^{13,14}$, R. Jeroen Vermeulen ${ }^{15}$, Esko J. Wiltshire ${ }^{16}$, Janice M. Fletcher ${ }^{17}$, Barry Lewis ${ }^{18}$, Gareth Baynam ${ }^{19,20}$, Carolyn Ellaway ${ }^{21,22}$, Shanti Balasubramaniam ${ }^{21}$, Kaustuv Bhattacharya ${ }^{21,22}$, Mary-Louise Freckmann ${ }^{23}$ Susan Arbuckle ${ }^{24}$, Michael Rodriguez ${ }^{25}$, Ryan J. Taft ${ }^{26}$, Simon Sadedin ${ }^{1,7}$, Mark J. Cowley ${ }^{27,28,29}$, André E. Minoche ${ }^{29}$, Sarah E. Calvo ${ }^{30,31,32}$, Vamsi K. Mootha ${ }^{30,31,32}$, Michael T. Ryan ${ }^{5}$, Yasushi Okazaki ${ }^{3}$, David A. Stroud ${ }^{4}$, Cas Simons ${ }^{1,33}$, John Christodoulou ${ }^{1,2,7,22}$ and David R. Thorburn ${ }^{1,2,7}$

${ }^{1}$ Murdoch Children's Research Institute, Melbourne, Australia, ${ }^{2}$ Department of Paediatrics, University of Melbourne, Melbourne, Australia, ${ }^{3}$ Diagnostics and Therapeutics of Intractable Diseases, Intractable Disease Research Center, Juntendo University, Graduate School of Medicine, Tokyo, Japan, ${ }^{4}$ Department of Biochemistry and Molecular Biology and Bio21 Molecular Science and Biotechnology Institute, University of Melbourne, Melbourne, Australia, ${ }^{5}$ Department of Biochemistry and Molecular Biology, Monash Biomedicine Discovery Institute, Monash University, Melbourne, Australia, ${ }^{6}$ Division of Genomic Medicine Research, Medical Genomics Center, National Center for Global Health and Medicine, Tokyo, Japan, ${ }^{7}$ Victorian Clinical Genetics Services, Melbourne, Australia, ${ }^{8}$ Department of Genetics, Yale School of Medicine, New Haven, USA, ${ }^{9}$ Australian Genome Research Facility Ltd, Melbourne, Australia, ${ }^{10}$ Department of Pediatrics \& Clinical Genomics, Saitama Medical University Hospital, Saitama, Japan, ${ }^{11}$ Department of Metabolism, Chiba Children's Hospital, Chiba, Japan, ${ }^{12}$ Ritchie Centre, Hudson Institute of Medical Research and Department of Paediatrics, Monash University; and Monash Newborn, Monash Children's Hospital, Melbourne, Australia, ${ }^{13}$ Child Neurology, Emma Children's Hospital, Amsterdam University Medical Centers, Vrije Universiteit and Amsterdam Neuroscience, Amsterdam, the Netherlands, ${ }^{14}$ Functional Genomics, Center for Neurogenomics and Cognitive Research, Vrije Universiteit and Amsterdam Neuroscience, Amsterdam, the Netherlands, ${ }^{15}$ Department of Neurology, Maastricht University Medical Center, Maastricht, the Netherlands, ${ }^{16}$ Department of Paediatrics and Child Health, University of Otago Wellington and Capital and Coast District Health Board, Wellington, New Zealand, ${ }^{17}$ Department of 
Genetics and Molecular Pathology, SA Pathology, Adelaide, Australia, ${ }^{18}$ Department of Clinical Biochemistry, PathWest Laboratory Medicine Western Australia, Perth, Australia, ${ }^{19}$ Western Australian Register of Developmental Anomalies and Genetic Services of Western Australia and King Edward Memorial Hospital for Women Perth, Perth, Australia, ${ }^{20}$ Telethon Kids Institute and School of Paediatrics and Child Health, The University of Western Australia, Perth, Australia, ${ }^{21}$ Genetic Metabolic Disorders Service, Sydney Children's Hospital Network, The Children's Hospital at Westmead, Sydney, Australia, ${ }^{22}$ Disciplines of Genomic Medicine and Child and Adolescent Health, Sydney Medical School, University of Sydney, Sydney, Australia, ${ }^{23}$ Clinical Genetics, The Canberra Hospital, Canberra, Australia, ${ }^{24}$ Department of Histopathology, The Children's Hospital at Westmead, Sydney Children's Hospital Network, Sydney, Australia, ${ }^{25}$ Discipline of Pathology, School of Medical Sciences, The University of Sydney, Sydney, Australia, ${ }^{26}{ }^{2}$ llumina, Inc, San Diego, USA, ${ }^{27}$ Children's Cancer Institute, Kensington, Australia, ${ }^{28} \mathrm{St}$ Vincent's Clinical School, UNSW Sydney, Sydney, Australia, ${ }^{29}$ Kinghorn Centre for Clinical Genomics, Garvan Institute of Medical Research, Sydney, Australia, ${ }^{30}$ Broad Institute, Cambridge, USA, ${ }^{31}$ Howard Hughes Medical Institute and Department of Molecular Biology, Massachusetts General Hospital, Boston, USA, ${ }^{32}$ Harvard Medical School, Boston, USA and ${ }^{33}$ Institute for Molecular Bioscience, The University of Queensland, Brisbane, Australia

Background: Mitochondrial disorders comprise $>350$ genetic conditions. In $\sim 50 \%$ of suspected cases, no molecular diagnosis is obtained, partly due to some chromosomal regions being refractory to standard genomic analyses. Aims: One challenging region is the ATAD3 locus, comprising 3 highly homologous tandemly arrayed genes (ATAD3C, $A T A D 3 B$ and $A T A D 3 A$ ), encoding mitochondrial proteins implicated in cholesterol metabolism, mitochondrial replication and morphology. Recessive deletions or dominant duplications cause lethal perinatal mitochondrial disorders characterized by pontocerebellar hypoplasia or cardiomyopathy, respectively. We therefore considered ATAD3 in unsolved cases referred for diagnostic mitochondrial investigations. Methods: Whole genome and exome sequencing; microarrays; long read sequencing; RNA studies; quantitative proteomics; biochemical analyses. Results: We identified 17 individuals from 16 unrelated families with cardiomyopathy, persistent lactic acidemia, and frequently corneal clouding or cataracts due to recurrent de novo ATAD3 duplications (Frazier et al., Med, June 2020). The six different $68 \mathrm{~Kb}$ duplications were identifiable by genomic sequencing, but usually missed on microarray. Long read genome sequencing confirmed the genomic architecture. The duplications all generated an identical chimeric $A T A D 3 A / A T A D 3 C$ protein, confirmed by RNA studies and quantitative proteomics. This chimeric protein appeared to act dominantly, causing altered $A T A D 3$ complexes and a striking reduction in mitochondrial complex I in heart tissue. Since publication, development of targeted clinical testing for ATAD3 duplications has identified three further affected individuals. Conclusion: In our experience, the ATAD3 locus has emerged as the most common cause of lethal perinatal mitochondrial disease but the repetitive sequence at the locus means diagnoses may be missed by current genomic strategies.

\section{Using Implementation Science Strategies to Promote the Appropriate Offering of Reproductive Genetic Carrier Screening: Mackenzie's Mission}

Stephanie Best ${ }^{1,2}$, Janet C Long ${ }^{1}$, Tahlia Theodorou ${ }^{1}$, Sarah Hatem ${ }^{1}$, Samantha Edwards ${ }^{3,4}$, Kirsten Boggs ${ }^{2,5,6}$ and Jeffrey Braithwaite ${ }^{1}$

${ }^{1} \mathrm{AlHI}$, Macquarie University, Melbourne, Australia, ${ }^{2}$ Australian Genomics Health Alliance, Australia, ${ }^{3}$ Centre for Medical Research, University of Western Australia, Perth, Australia, ${ }^{4}$ Harry Perkins Institute for Medical Research, Perth, Australia, ${ }^{5}$ Sydney Children's Hospital Network — Randwick, Sydney, Australia and ${ }^{6}$ Sydney Children's Hospital Network — Westmead, Sydney, Australia
To date there has been little attention paid to healthcare practitioners offering reproductive genetic carrier screening (RGCS). This group is essential to consider; without their engagement consumers will not readily be able to gain access to screening. Aim: To apply an implementation science theoretical approach in order to design strategies to promote appropriate offering of RGCS. We used a mixed methods strategy: 1) the validated Influences on Patient Safety Behaviours Questionnaire (IPSBQ) that uses behavior change theory (Theoretical Domains Framework; TDF) for healthcare practitioners (GPs, genetic specialists, midwives) undertaken at the programme onboarding education, to identify perceived barriers to offering RGCS, followed by; 2$)$ a focus group $(n=1)$ and interviews $(n=3)$ with all the programme genetic counselors $(n=8)$ to identify potential interventions and measures. Survey data were analyzed using the pre-designed IPSBQ tool. Qualitative data were analyzed using content analysis. In total, 274 healthcare practitioners completed the survey (response rate of 70\%). Dominant TDF domains identified were skills, environment and resources, and motivation. Genetic counselors identified a range of potential interventions, drawing on their experience with healthcare practitioners in the programme, including an education video (skills), group sessions (motivation/social influences) and waiting room posters (consumer-led/environment and resources) that we matched to the TDF domains identified. The identified codesigned strategies are currently being trialled. Using theory to match barriers to implementation with strategies to overcome them promotes a targeting of interventions. A theory informed process leads to the avoidance of a scatter-gun approach to promoting implementation, potentially reducing wasting of resources.

\section{Ultra-Rapid Diagnosis of Focal Congenital Hyperinsulinism in a Critically Ill Infant}

Crystle Lee ${ }^{1}$, Amber Boys ${ }^{1}$, Anna Le Fevre ${ }^{1}$, Michelle G. de Silva ${ }^{1,2,3}$, Belinda Chong ${ }^{1}$, Sebastian Lunke $\mathrm{k}^{1,3}$ and Zornitza Stark ${ }^{1,2,3}$

${ }^{1}$ Victorian Clinical Genetics Services, Melbourne, Australia, ${ }^{2}$ Australian Genomics Health Alliance, Melbourne, Australia and ${ }^{3}$ The University of Melbourne, Melbourne, Australia

Background: The Acute Care Genomics study provides ultra-rapid genomic testing for critically ill pediatric patients with suspected monogenic disorders, with time to result of less than 5 calendar days and the aim of improving clinical outcomes through early diagnosis and guiding treatment. Methods: Ultra-rapid trio WGS was performed on DNA extracted from blood in a critically ill neonate who presented with hypoglycemic seizures within hours of birth and a clinical diagnosis of hyperinsulinaemic hypoglycemia, unresponsive to treatment with diazoxide. Subsequent SNP-microarray analysis was performed on resected pancreatic tissue. Results: A paternally inherited heterozygous pathogenic loss-of-function variant in ABCC8, c.3776dup; p.(Val1260Glyfs*147), was identified within 71 hours of sample receipt, consistent with a molecular diagnosis of focal congenital hyperinsulinism (CHI), and directing management towards surgical resection. SNP-microarray analysis on resected pancreatic tissue detected mosaic segmental uniparental isodisomy (UPiD) of the $11 \mathrm{p} 15$ region, encompassing ABCC8. Discussion: The genetic basis of ABCC8-related congenital hyperinsulinism is complex, with both dominant and recessive modes of inheritance observed in families. The rapid identification of a loss-of-function variant inherited from an asymptomatic parent promptly directed management towards surgical resection of focal pancreatic lesion, and subsequent confirmation of loss of 
heterozygosity in the 11 p15 region, consistent with somatic bi-allelic disease. As focal CHI results from two genetic events occurring independently, the overall recurrence risk is estimated to be 1 in 1200 . This report highlights the value of ultra-rapid genomic testing in facilitating early diagnosis and enabling precision management in critically ill babies with suspected monogenic diseases.

\section{'Work That Will Make You Proud'. Clinicians' and Scientists' Experiences Two Years Into a Precision Medicine Trial for Childhood Cancer Patients}

Emily Hazell $^{1}$, Kate Hetherington ${ }^{2,3}$, Claire Elizabeth Wakefield ${ }^{2,3}$ and Chris Jacobs ${ }^{1}$

${ }^{1}$ Graduate School of Health, Discipline of Genetic Counselling, University of Technology, Sydney, Ultimo, Australia, 'School of Women's and Children's Health, UNSW, Sydney, Australia and ${ }^{3}$ Behavioural Sciences Unit, Kids Cancer Centre, Sydney Children's Hospital, Sydney Australia

Background: The PRecISion Medicine for children with cancer (PRISM) trial is the first Australia-wide precision medicine trial for children and adolescents with high-risk cancer. PRISMIMPACT, a psychosocial sub-study of PRISM, explored clinicians' and scientists' experiences two years into the trial. Clinicians and scientists work closely to determine whether treatment recommendations can be made for each PRISM patient on the basis of the data collected about each child's tumor. Understanding their views and experiences of the trial is therefore crucial for implementation. Methods: We interviewed 29 clinicians (oncologists, geneticists/ genetic counselors) and 10 scientists (bioinformaticians, research assistants/officers and laboratory scientists) who were part of PRISM. We transcribed interviews verbatim and analyzed the data using thematic analysis. Results: Three main themes were identified: personal growth and satisfaction, feeling the pressure of responsibility and balancing hopes and expectations. Participants were excited about making a direct impact on families, the opportunity to learn, and collaborations formed through this new way of working. The main challenge reported was the urgency around turnaround times to deliver results. Scientists reported emotional pressures they had not previously experienced, and clinicians described challenges regarding deciding whether or not to recommend certain treatments to patients with a limited evidence-base. Discussion/conclusion: Clinicians and scientists are excited about the potential for precision medicine. Addressing challenges by providing scientists with emotional support and streamlining the reporting of results from the laboratory to the clinic would help to ensure these groups are well prepared for this new way of working.

\section{Network-Based Nonparametric Analysis to Identify Genetic Modifiers of Niemann-Pick Type C Disease}

Eliatan Niktab ${ }^{1}$, Stephen Sturley ${ }^{2}$, Ingrid Winship ${ }^{3,4}$, Andrew Munkacsi ${ }^{1}$ and Mark Walterfang 3,4

${ }^{1}$ Victoria University of Wellington, New Zealand, ${ }^{2}$ Barnard College at Columbia University, New York, USA, ${ }^{3}$ Royal Melbourne Hospital, Melbourne, Australia and ${ }^{4}$ University of Melbourne, Melbourne, Australia

Relative to the extensive success of genome and exome sequencing in identifying high-penetrance disease-causing variants, there has been little success identifying variants that modify these disease-causing genes and subsequently the onset and progression of Mendelian diseases. Identifying modifiers is particularly difficult since modifiers, unlike causal variants, are not necessarily rare variants given their action is epistatic with the disease-causing gene. Investigation of epistasis has only recently become feasible by utilizing convolutional neural networks and high-dimensional datasets comprised of genome sequences and clinical records. Niemann-Pick type $\mathrm{C}$ (NP-C) disease, a rare monogenic disease, is one of more than 6,000 Mendelian diseases for which there is no cure. Most NP-C patients with the NPC1 gene mutation are diagnosed as late infants and die before or during adolescence suffering visceral and neurodegenerative symptoms, yet survival of some to adulthood provides a testbed for elucidating genes that alleviate the primary mutation. Here we report investigation of genetic variants that modify the causal gene of NP-C disease and ultimately regulate its onset and progression in individuals. We sequenced a cohort of early-onset discordant affected NP-C siblings, a cohort of late-onset NP-C patients, and trained our deep learning model on a dataset comprised of genome sequences of $>100,000$ persons (healthy or diagnosed with other diseases). Then we developed an analytical tool that identifies genome-wide interactions via scripts that control for confounding factors using data harmonization and modularity-based clustering. This tool mitigates the statistical challenge of sample sizes inherent to current GWAS methodology.

\section{Warts and All: Lessons from Scaling Up Implementation of Ultra-Rapid Genomic Testing in Critically Ill Children}

Stephanie Best ${ }^{1,2}$, Helen Brown ${ }^{3}$, Sebastian Lunke ${ }^{4,5}$, Chirag Patel ${ }^{6}$, Jason Pinner ${ }^{7}$, Christopher Barnett ${ }^{8}$, Meredith Wilson ${ }^{9,10}$, Sarah A Sandaradura ${ }^{9,11}$, Belinda MaClaren ${ }^{2,5}$, Gemma Brett ${ }^{4,5}$, Jeffrey Braithwaite ${ }^{1}$ and Zornitza Stark ${ }^{2,4,5}$

${ }^{1} \mathrm{AlHI}$, Macquarie University, Melbourne, Australia, ${ }^{2}$ Australian Genomics Health Alliance, Australia, ${ }^{3}$ Deakin University, Melbourne, Australia, ${ }^{4}$ Victorian Clinical Genetics Services, Melbourne, Australia, ${ }^{5}$ The University of Melbourne, Melbourne, Australia, ${ }^{6}$ Genetic Health Queensland, Royal Brisbane and Women's Hospital, Brisbane, Australia, ${ }^{7}$ Sydney Children's Hospitals Network, Sydney, Australia, ${ }^{8}$ Paediatric and Reproductive Genetics Unit, South Australian Clinical Genetics Service, Adelaide, Australia, ${ }^{9}$ Sydney Children's Hospitals Network, Sydney, Australia, ${ }^{10}$ Discipline of Genomic Medicine, University of Sydney, Sydney, Australia and ${ }^{11}$ Discipline of Child and Adolescent Health, University of Sydney, Sydney, Australia

Rapid genomic testing can influence the management of critically ill children with genetic conditions. Despite growing evidence of efficacy, implementation to date has been mostly limited to single academic centres, and most of the reported outcomes restricted to diagnostic yield and clinical utility. Aim: To apply implementation science principles and theory to examine major influences on scaling up an ultra-rapid genomics program to national level, identifying future implementation strategies for sustainable services. Key professionals delivering a multisite ultra-rapid genomics program in pediatrics (clinical geneticists, genetic counselors, intensivists, laboratory scientists) were invited $(N=61)$ to participate in semi-structured interviews by the lead researcher. Transcripts were analyzed by two researchers using the Consolidated Framework for Implementation Research. Participants $(n=32)$ reported common themes including the importance of networks and relationship building, leadership, culture and the relative advantage afforded by ultrarapid genomic testing in the care of critically ill children. While clinical geneticists focused on intervention characteristics and the fit with patient-centred care, intensivists emphasized the importance of access to knowledge, in particular from clinical geneticists. The relative advantage of ultra-rapid genomics and trust in consistent and transparent delivery were significant in creating engagement at initial implementation, with appropriate resourcing highlighted as important for longer term sustainability. We have used theory informed approaches to identify intervention strategies to support 
the wider implementation of rapid genomic testing. While some approaches may be universal, there is a need to tailor support by professional role and implementation phase, to maximize the potential of ultra-rapid genomics to improve patient care.

\section{Studying Chromosome Conformation in Health and Disease With Arima Genomics Technologies}

Frank Boellmann ${ }^{1}$ and Anthony Schmitt ${ }^{1}$

${ }^{1}$ Arima Genomics, San Diego, USA

Chromosome conformation capture technologies, such as $\mathrm{Hi}-\mathrm{C}$, HiChIP, and Capture-HiC, are powerful approaches for profiling $3 \mathrm{D}$ genome structure and providing valuable insights into the mechanisms of gene regulation in human disease. Disease-specific chromosome folding patterns have been implicated across numerous human pathologies, such as cancer and have been valuable for the functional interpretation of non-coding disease associated variants (GWAS). The ability to physically connect extremely distant regions of the same DNA molecule can also be leveraged for applications such as scaffolding de novo assemblies of human genomes or the discovery and phasing of sequence and structural variants. Our reproducible and flexible kit platform for the study of genome-wide (Hi-C) and targeted (HiChIP, Capture-HiC) genome structures is based on our core proximity ligation chemistry. The 6-hour protocol improves analytical sensitivity through rapid, multiple restriction enzyme Hi$\mathrm{C}$ chemistry. This significantly improved technology detects more chromatin folding features, such as chromatin loops, from significantly reduced sequencing depth. The Arima-HiC kit has been widely validated through scientific publications across research domains including oncology, cardiology, neurobiology, and immunology. The optimized targeted chromosome conformation capture technologies from Arima Genomics significantly enhance the ability of clinical and translational researchers to study pathological mis regulation of chromosome conformation in fine detail and at reduced costs. We will present our customer validated $\mathrm{H} 3 \mathrm{~K} 27 \mathrm{ac}$ and $\mathrm{H} 3 \mathrm{~K} 4 \mathrm{me} 3 \mathrm{HiChIP}$ protocols, demonstrating reproducible detection of long-range interactions at active promoters as well as data from high-resolution (500bp) Capture-HiC experiments targeting oncogenes and tumor suppressors in a panel of 10 cancer and non-cancer samples.

\section{Variant Atlas: A Scalable Cohort Analysis and Data Sharing Platform for the Australian Genomics Clinical Flagships}

Dmitry Degrave ${ }^{3}$, Andre Hermanto ${ }^{3}$, Shyamsundar Ravishankar ${ }^{3}$, Joe Copty ${ }^{3}$, Shane Husson ${ }^{3}$, Marie-Jo Brion ${ }^{1,2}$ and Warren Kaplan ${ }^{3}$

${ }^{1}$ QIMR Berghofer Medical Research Institute, Brisbane, Australia, ${ }^{2}$ Australian Genomics Health Alliance, Melbourne, Australia and ${ }^{3}$ Kinghorn Centre for Clinical Genomics, Garvan Institute of Medical Research, Sydney, Australia

Background: Precision medicine programs rely on genomic sequencing of disease cohorts. However, effective use of genomic data is challenging due to the large computational resources, data storage requirements, and the millions of variants present per participant. Aim: To design a platform that would enable easy interrogation of genome cohorts of any size, support streamlined, ethical data access and suit the needs of diverse users including clinicians, bench scientists and bioinformaticians. Methods: A scalable genotype-phenotype database ('Variant Atlas') was built, as a controlled-access, webbased interface, for exploring a cohort's genomic and phenotypic data with interactive queries and visualizations. The platform combines participants' genomic data, and links to clinical phenotypes. The platform enables researchers to explore genomic characteristics at a cohort-level, query specific genes or variants of interest, or discover variants in patient subgroups with particular clinical features. Results: Variant Atlas launched in August and is making whole genome and exome data available for queries, from rare disease and cancer cohorts of the Australian Genomics Health Alliance. Aggregated data queries are available to all researchers and clinicians via registered access. Patient-level clinical data queries are available via restricted access to the cohort teams and clinicians. Variant Atlas currently has over 70 registered users and will complete integration for 12 rare disease and cancer cohorts. Conclusion: Variant Atlas (variantatlas.org.au) has powerful capabilities for housing and facilitating analyses of diverse Australian Genomics cohorts and supports a range of data sharing use cases.

\section{International Expanded Carrier Screening Experience}

Nicole Schonrock ${ }^{1}$, Dana Neitzel ${ }^{1}$, Julia Wilkinson ${ }^{1}$, Asia Mitchell ${ }^{1}$, Nicole Faulkner ${ }^{1}$ and Swaroop Aradhya ${ }^{1}$

${ }^{1}$ Invitae, San Francisco, USA

Background: Gene panels for carrier screening are rapidly expanding in size and clinical use. Professional guidelines vary among countries in which genes should be included on panels and in the recommended testing strategy for couples. The aim of this study was to assess our carrier screening experience including clinicians worldwide. Methods: Screening was performed for individual patients for up to 301 genes. Genes could be ordered in pre-curated panels (3, 46, or 288 genes), as a single test or as customized panels. Thirteen common and/or variable genes were available as an addon to any panel. Ordering patterns and positive rates were assessed. Results: We evaluated 99,112 individuals referred from $>3,700$ clinicians worldwide. Testing was ordered concurrently for both partners for $\sim 60 \%$ of samples. The largest pre-curated panel was ordered most frequently across all clinician types and made up $57 \%$ of all orders. The overall positive rate was $51 \%$, with a carrier couple rate of $15 \%$ (including variable and mild disorders). Of all positives, $\sim 31 \%$ were positive for 1 condition and $\sim 23 \%$ for $\geq 2$ conditions. Conclusions: Amid wide variability in carrier screening utilization, it was evident from this study that many clinicians opt for large carrier screen panels (even those including frequent/variable disorders) and that many clinicians order testing on both members of a couple concurrently. Additional investigation is needed to understand the decisions behind these patterns within and between US and international clinicians and to investigate how these results are used by patients and their providers.

\section{Sponsored Testing Programs: An Emerging Paradigm to Accelerate Diagnosis and Precision Care of Individuals With Rare Genetic Diseases}

Nicole Schonrock ${ }^{1}$, Nandor Roczo ${ }^{1}$, Dianalee McKnight ${ }^{1}$, Britt Johnson ${ }^{1}$, Andrew Wilcock ${ }^{1}$, Rebecca Truty ${ }^{1}$, Gautam Mehta ${ }^{1}$, Daniel Anderson ${ }^{1}$ and Swaroop Aradhya ${ }^{1}$

${ }^{1}$ Invitae, San Francisco, USA

Patients suffering from rare hereditary diseases have benefited from the recent surge in development of targeted therapies. There are numerous challenges with diagnosing and identifying individuals eligible for therapies, including cost of and access to genetic testing, delayed time to diagnosis, and delayed access to treatment. 
Effective partnerships have been formed among clinicians, genetic testing laboratories, and biopharmaceutical companies to help patients receive therapies sooner. Biopharma companies sponsor genetic testing for individuals meeting eligibility criteria, helping to identify those with specific molecular diagnoses for which therapies are available. This study aimed to evaluate the clinical utility and effectiveness of sponsored programs in a large clinical cohort. From December 2016 to May 2020, 60,215 unrelated probands and 1311 family members obtained no-cost genetic testing through 17 sponsored testing programs across diverse clinical areas. Overall, 11,492 unrelated individuals received a molecular diagnosis or likely molecular diagnosis, $68 \%$ of which were in genes that were not of primary interest to the sponsor. The highest diagnostic yields were for tests related to ophthalmology (40\%), inherited metabolic disorders (31\%), and cardiology (18\%). Of those receiving diagnoses, 376 received them after reflex testing to a larger panel. Among the 1311 family members who underwent targeted variant testing, 383 received molecular diagnoses. These results demonstrate that sponsored testing programs provide an effective approach for eliminating barriers to genetic testing, identifying individuals who are eligible for therapies, identifying differential diagnoses, and facilitating testing for relevant family members.

\section{Increasing Diagnostic Yield for Patients With Primary Immunodeficiency}

Elvira Zilliacus ${ }^{1}$, Anni Niskakoski², P Kokkonen ${ }^{2}$, Inka Saarinen ${ }^{2}$, Johanna Sistonen ${ }^{2}$, Heidi Junnila², Annakarin Kere ${ }^{2}$, Margarita Andreevskaya², Mikko Muona², Janica Djupsjobacka², Lotta Koskinen ${ }^{2}$, Hatice Duzkale ${ }^{2}$, Samuel Myllykangas², Juha Koskenvuo ${ }^{2}$ and T-P Alastalo ${ }^{2}$

${ }^{1}$ Blueprint Genetics, Sydney, Australia and ${ }^{2}$ Blueprint Genetics, Helsinki, Finland

Background: Finding the genetic diagnosis for patients with suspicion of primary immunodeficiency (PID) is becoming increasingly important in the management of primary immunodeficiency and estimating the risk for family members. Simultaneous sequence and copy number variant analysis combined with customized analysis for difficult to sequence regions in specific genes such as NCF1 improves the diagnostic yield in patients suspected for PID. Methods: Approximately 1700 patients suspected of PID and referred for genetic testing at Blueprint Genetics between 2018-19 were retrospectively reviewed. Patients were tested with Blueprint Genetics next generation sequencing Immunology Panels. Results: Diagnostic yield including all immunology related panels was $14.9 \%$ (253/1698). The diagnostic yield was highly variable between the different panels; diagnostic yield for primary immunodeficiency panel was $13.3 \%$ (138/1036), whereas for severe combined immunodeficiency panel it was $46.7 \%$ (7/15). The diagnostic yield for chronic granulomatous disease panel is high $(10 / 21,48 \%)$ although analysis of the NCF1 gene included in this panel is complicated by two highly homologous pseudogenes NCF1P and NCF1C. 296 diagnostic sequence variants were reported, of which 234 (79\%) were unique, and only 7 diagnostic variants were reported over 3 times. Copy number variants, including deletions and duplications, were reported for 19 patients from 17 different genes. Conclusion: We constantly increase the diagnostic yield for PIDs by improving the sequencing technology, updating the panels with new genes discovered related to PID, and finding diagnoses from difficult to sequence regions and regions with high homology.

\section{Characteristics and Frequency of Copy Number Variants Across Medical Specialties}

Elvira Zilliacus ${ }^{1}$, Kim Gall $^{1}$, Inka Saarinen ${ }^{1}$, Julie Hathaway ${ }^{1}$, Juha Koskenvuo ${ }^{1}$ and T-P Alastalo ${ }^{1}$

${ }^{1}$ Blueprint Genetics, Helsinki, Finland

Background: As next-generation sequencing (NGS) has become more sensitive to detecting copy number variants (CNVs), their prevalence across medical specialties is becoming clearer. are an important cause of hereditary disease. However, an NGS assay's ability to detect various sized CNVs is highly dependent upon the testing platform and bioinformatics pipeline. Methods: A retrospective review of 10,688 deidentified genetic test results, performed on a whole-exome sequencing platform for a variety of clinical indications, was undertaken to assess the proportion, frequency and characteristics of diagnostic CNVs identified on a whole-exome sequencing platform in patients referred for clinical genetic testing. Results: Diagnostic CNVs were identified in $3.8 \%$ of all tests (401/ $10,688)$ and made up $9.8 \%$ of all diagnostic variants $(401 / 4,093)$. Most (91.5\%) diagnostic CNVs were deletions. CNVs ranging from 1-exon deletions/duplications to multiple-gene deletions/duplications were identified across almost all medical categories. Importantly, $24.2 \%$ of CNVs were $\leq 2$ exons in size. Diagnostic CNVs made up the highest proportion of all diagnostic findings in whole exome tests (45.3\%), pulmonology (33.3\%), and earnose-throat (20.1\%). Diagnostic CNVs made up the smallest proportion of all diagnostic findings in the ophthalmology (6.9\%), endocrinology (6.9\%), and cardiology (6.0\%) categories. Conclusions: Diagnostic CNVs made up approximately $10 \%$ of all diagnostic variants identified on this whole-exome sequencing platform. Our results suggest that small CNVs may account for a significant proportion of disease-causing CNVs and demonstrate the ability of this comprehensive genetic testing approach to detect CNVs $\leq 2$ exons in size, which may improve diagnostic yield.

\section{Jumping From Class 3-to-5: Impact of Functional RNA Diagnostics in Danon Disease With Gonadal Mosaic LAMP2 Splicing Variant}

Ari Horton ${ }^{1,2,3,4}$, Adam Bournazos ${ }^{5,6}$, Fiona Cunningham ${ }^{1}$, Matthew Regan ${ }^{1,3}$, Sarah Hope ${ }^{2,3,4}$, Ivan Macciocca ${ }^{7}$, Emma Krzesinski ${ }^{1,3}$, Sandra T. Cooper ${ }^{5,6,8}$ and Matthew F. Hunter ${ }^{1,3}$

${ }^{1}$ Monash Genetics, Monash Health, Melbourne, Australia, ${ }^{2}$ Monash Heart, Monash Health, Melbourne, Australia, ${ }^{3}$ Department of Paediatrics, Monash University, Melbourne, Australia, ${ }^{4}$ Monash Cardiovascular Research Centre, Melbourne, Australia, ${ }^{5}$ Kids Neuroscience Centre, Children's Hospital Westmead, Sydney, Australia, ${ }^{6}$ Faculty of Medicine and Health, University of Sydney, Sydney, Australia, ${ }^{7}$ Victorian Clinical Genetics Services, Murdoch Children's Research Institute, Melbourne, Australia and ${ }^{8}$ Children's Medical Research Institute, Sydney, Australia

LAMP2 variants cause Danon disease, an X-Linked dominant lysosomal disorder (OMIM \#300257) characterized by hypertrophic cardiomyopathy (HCM), rhythm disturbance, mild intellectual disability and skeletal myopathy. ACMG classification guidelines struggle with splice variants of uncertain significance. RNA splicing studies are new in cardiac genomics but can be critical in confirming pathogenicity. Studies demonstrating cost-effectiveness will further support its introduction clinically. Case Report: A 6-month-old boy was found incidentally to have severe concentric HCM with 
preserved systolic function following review for patent ductus arteriosus. Initial investigations to determine the aetiology were nondiagnostic. By 12 months he had developed subtle skeletal myopathy with raised $\mathrm{CK}(1152 \mathrm{H})$ and developmental delay. Results: Genomic testing identified a LAMP2 splicing-variant of uncertain significance (VUS) (ChrX GRCh37: g.119576451T>A, NM_013995.2: c. $928+3 \mathrm{~A}>\mathrm{T}$ ), according to ACMG guidelines. The variant was absent from a large population database (gnoMAD v2.1) and had not been previously reported. It segregated with disease in an affected older sibling. The mother was negative for the LAMP2 c.928+3A>T variant, suggesting gonadal mosaicism. Functional testing of LAMP2 pre-mRNA splicing using blood from both siblings established that c.928+3A > T variant induced exon-7 skipping (r.865_928del) with no evidence of residual normal splicing. Exon 7 skipping induces a frameshift (p.Lys289Phefs*26) with clear, deleterious consequences for the encoded protein, enabling re-classification to class 5 (pathogenic). Conclusion: RNA diagnostics are increasingly used clinically to clarify pathogenicity. Increasing availability of adjunct supportive studies will lead to improved diagnostic rates and patient outcomes from genomic testing.

\section{METABOLIC AND DIET DISEASE MANAGEMENT}

\section{Biotin-Thiamine-Responsive Basal Ganglia Disease: Case Report and Follow-Up of a Patient With Poor Compliance}

Sharmila Kiss ${ }^{1}$ and Heidi Peters ${ }^{1}$

${ }^{1}$ The Royal Children's Hospital, Melbourne, Melbourne, Australia

Background: Biotin-thiamine-responsive basal ganglia disease is a rare treatable autosomal recessive metabolic disorder caused by mutations in the SLC19A3 gene. It is characterized by subacute encephalopathy with confusion, convulsions, dysarthria, and dystonia. The disorder is completely reversible if treated early with biotin and thiamine, and can progress to quadriparesis and death if left untreated. Results: We report a case of an 11-year, 3-month-old girl who was diagnosed with biotin-thiamine-responsive basal ganglia disease at 6 years of age through cascade screening. Her sibling had presented with an acute neurological event at 5 months of age. The patient was asymptomatic but was treated with biotin and thiamine, and the family instructed to continue medications lifelong. When she was 11 years old, her supplements were stopped. After 4 weeks the patient developed vertical diplopia and headaches. She was admitted and commenced on high dose thiamine and biotin. Brain magnetic resonance imaging was normal at the time of admission. Ophthalmology review showed cranial nerve IV palsy. Further follow up showed improvement in her ophthalmology examination. The family was further educated about the importance of strict compliance. Conclusion: Patients with biotin-thiamine-responsive basal ganglia disease should remain on lifelong treatment with thiamine and biotin.

\section{Emergency Management of High Phenylalanine Levels in an Unplanned Pregnancy Using Enteral Feeding in an Outpatient Setting: A Case Report}

Kate Lefebure ${ }^{1}$, Claire Rutledge ${ }^{1}$, Anne-Marie Desai ${ }^{1}$, Kaye Quick ${ }^{1}$, Christine Fischer ${ }^{1}$, Julie Panetta ${ }^{1}$, Tim Fazio ${ }^{1,2}$ and Geradus de Jong,

${ }^{1}$ Metabolic Disease Unit, The Royal Melbourne Hospital - Melbourne Health, Melbourne, Australia and ${ }^{2}$ The University of Melbourne, Melbourne Medical School, Department of Medicine and Radiology, Royal Melbourne Hospital, Melbourne, Australia
Background: High maternal blood phenylalanine (phe) levels are known to be teratogenic to the developing foetus, with early phe control correlated with improved fetal outcome. In cases where a pregnancy is unplanned to a mother with PKU, an emergency management plan is required to lower blood phe levels. To date there are no documented cases of administration of an emergency protocol using nasogastric tube (NGT) feeding in an outpatient setting. Research Question: Can outpatient NGT feeding be used to stabilize blood phe levels as emergency management of an unplanned PKU pregnancy? Methods: Dry blood spot phe levels and dietary management were retrieved from a single patient's medical record. Costing data was obtained from the clinical costing unit. Results: Timely outpatient insertion of a NGT facilitated the implementation of proteinfree, calorie-rich emergency protocol, resulting in rapid reduction of plasma phe from 1160 umol/L to $<250 \mathrm{umol} / \mathrm{L}$ in six days. NGT displacement requiring reinsertion, was one minor complication recorded. A prior five-day admission for NGT insertion and emergency phe stabilization cost approximately AUD $\$ 5700$, compared to an AUD $\$ 660$ in an outpatient setting. A reduction in indirect costs was noted with the patient and her partner able to continue to work and care for their child, avoiding additional child care costs. Nonfinancial benefits include minimal separation from first child and husband, reduced anxiety, limited exposure to infection, improved sleep and improved compliance. Discussion/Conclusion: In carefully selected patients, NGT feeding in the outpatient setting should be considered to optimize patient compliance, comfort and reduce overall healthcare costs.

\section{Clinical Turnaround After Givosiran Treatment for Refractory Acute Intermittent Porphyria}

Timothy Fazio ${ }^{1,2}$, Julie Panetta ${ }^{1}$, Christine Fischer ${ }^{1}$, Kaye Quick ${ }^{1}$ and Gerard de Jong ${ }^{1,2}$

${ }^{1}$ Statewide Adult Metabolic Diseases Unit, Royal Melbourne Hospital, Melbourne, Australia and ${ }^{2}$ The University of Melbourne, Melbourne Medical School, Department of Medicine and Radiology, Royal Melbourne Hospital, Melbourne, Australia

We report on a 40-year-old woman admitted for severe abdominal pain on the background of acute intermittent porphyria refractory to regular medical treatment. Diagnosed aged 28, symptoms initially responded to glucose therapy. The increasing frequency of attacks soon necessitated increasing doses of haemarginate for symptom control; goserelin did not reverse this trend. Despite weekly prophylactic haemarginate infusions, treatment progressed to frequent emergency admissions for multi-day courses of haemarginate, eventually culminating in 513 inpatient days over 91 hospital admissions. Because of her extremely poor quality of life, chronic dependency on opioids for pain control, superior vena cava obstruction secondary to haemarginate infusions, critically limited venous access, and increasing iron overload consequential to haemarginate infusions, she was referred for consideration of liver transplantation. During this 47 -day admission, the patient was treated with repeated 4-5 day courses of haemarginate. The urine porphobilinogen/creatinine ratio peaked at $51.2 \mathrm{umol} / \mathrm{mmol}$ and fell to $6.7 \mathrm{umol} / \mathrm{mmol}$, with rebound symptoms and biochemical marker elevations within days of treatment. At this point, givosiran treatment became available under an extended access program and was commenced at $2.5 \mathrm{mg} / \mathrm{kg}$ monthly. In the five months on givosiran, her quality of life has improved remarkably; symptoms are reduced, she has come off all analgesics, not required hospital admissions, not required further haemarginate infusions and is 
no longer under consideration for transplantation. Conclusion: Givosiran has the potential to significantly improve the quality of life in patients with acute intermittent porphyria refractory to current medical therapies, reduce hospital admissions, and reduce dependence on analgesics.

\section{MOLECULAR GENETICS}

\section{Heterozygous Loss of WBP11 Function Causes Multiple Congenital Defects in Humans and Mice}

Ella Martin ${ }^{1}$, Annabelle Enriquez ${ }^{1,2}$, Duncan Sparrow ${ }^{1,2,3}$, David Humphreys ${ }^{1,2}$, Aideen Mclnerney-Leo ${ }^{4}$, Paul Leo ${ }^{5}$, Emma Duncan ${ }^{5}$, Kavitha lyer ${ }^{1}$, Joelene Greasby ${ }^{1}$, Ip Eddie, Eleni Giannoulatou ${ }^{1,2}$, Delicia Sheng ${ }^{1}$, Elizabeth Wohler ${ }^{6}$,

Clémantine Dimartino ${ }^{7}$, Jeanne Amiel ${ }^{7}$, Yline Capri ${ }^{8}$, Daphné Lehalle ${ }^{9}$, Adi Mory ${ }^{10}$, Yael Wilnai ${ }^{10}$, Yael Lebenthal ${ }^{11}$, Ali Gharavi ${ }^{12}$, Grażyna Krzemień ${ }^{13}$

Monika Miklaszewska ${ }^{14}$, Robert Steiner ${ }^{15}$, Cathy Raggio ${ }^{16}$, Robert Blank ${ }^{17}$, Hagit Baris Feldman ${ }^{10}$, Hila Milo Rasouly ${ }^{12}$, Nara Sobreira ${ }^{6}$, Rebekah Jobling ${ }^{18}$,

Christopher Gordon 7 , Philip Giampietro ${ }^{19}$, Sally Dunwoodie ${ }^{1,2}$ and Gavin Chapman ${ }^{1,2}$

${ }^{1}$ Victor Chang Cardiac Research Institute, Sydney, Australia, ${ }^{2}$ UNSW, Sydney, Australia, ${ }^{3}$ University of Oxford, Oxford, UK, ${ }^{4}$ The University of Queensland Diamantina Institute, Brisbane, Australia, ${ }^{5}$ Institute of Health and Biomedical Innovation, Queensland University of Technology, Brisbane, Australia, ${ }^{6} \mathrm{Johns}$ Hopkins University, Baltimore, USA, ${ }^{7}$ Paris Descartes-Sorbonne Paris Cité Université, Institut Imagine, Paris, France, ${ }^{8} \mathrm{Hôpital}$ Robert Debré, Assistance Publique Hôpitaux de Paris, Paris, France, ${ }^{9}$ Centre Hospitalier Intercommunal Créteil, Créteil, France, ${ }^{10}$ Tel Aviv Sourasky Medical Center, Tel Aviv, Israel, ${ }^{11}$ Sackler Faculty of Medicine, Tel Aviv University, Tel Aviv, Israel, ${ }^{12}$ Columbia University, New York, USA, ${ }^{13}$ Warsaw Medical University, Warsaw, Poland, ${ }^{14}$ Jagiellonian University of Cracow, Kraków, Poland, ${ }^{15}$ University of Wisconsin School of Medicine and Public Health, Madison, USA, ${ }^{16} \mathrm{Hospital}$ for Special Surgery, Pediatrics Orthopedic Surgery, New York, USA, ${ }^{17}$ Medical College of Wisconsin, Milwaukee, USA, ${ }^{18}$ The Hospital for Sick Children, Toronto, Canada and ${ }^{19}$ Robert Wood Johnson School of Medicine, New Brunswick, USA

Congenital malformations affect 3-6\% of live human births and are associated with higher rates of fetal demise. In $80 \%$ of cases, the underlying etiology is unknown. We hypothesized that there are uncharacterized genetic causes of malformations such as vertebral malformation (VM) and congenital heart disease. In order to identify additional genetic causes of VMs we exome-sequenced DNA from 45 trios and families with VM. Rare predicted pathogenic variants that segregated with disease were selected. In two unrelated patients with Klippel-Feil Syndrome, novel heterozygous stop gain variants in the WBP11 gene were identified. WBP11 encodes an evolutionarily conserved activator of splicing, which has not been previously linked to human disease. Ten additional patients from five families were identified via GeneMatcher, four families with heterozygous truncating WBP11 variants and one with a predicted pathogenic missense variant in WBP11. Although patient phenotypes varied, common malformations included cervical vertebral fusions, oesophageal atresia, kidney and heart defects. We generated a mouse model of WBP11 heterozygosity by CRISPR-Cas9 targeting. Mice heterozygous for a Wbp11 null allele were not found in the expected Mendelian ratio with many dying either late in gestation, postnatally or as adults. Defects of the axial skeleton, oesophagus and kidneys were found in Wbp11 heterozygous mice, similar to affected individuals, confirming WBP11 mutation as a cause of multiple congenital anomalies in humans and mice.

\section{Advancing the Diagnosis of Mitochondrial Diseases with Trio Genome Sequencing}

Rocio Rius $^{1,2}$, Lisa G. Riley ${ }^{3,4}$, Mark J. Cowley ${ }^{5,6}$, Velimir Gayevskiy ${ }^{5}$, Andre E. Minoche ${ }^{5}$, Clare Puttick ${ }^{5}$, Alison G. Compton ${ }^{1,2}$, Minal J. Menezes ${ }^{3,4}$, Kaustuv Bhattacharya ${ }^{4,7}$, David Coman ${ }^{8,9,10}$, Carolyn Ellaway $y^{4,11,12}$, Himanshu Goe ${ }^{13}$, Ian E. Alexander ${ }^{4,11}$, Louisa Adams ${ }^{4,11,12}$, Maina Kava ${ }^{14,15,16}$, Jacqui Robinson ${ }^{17}$, Carolyn M. Sue ${ }^{5,18}$, Shanti Balasubramaniam ${ }^{3,4}$, Nicole J. Van Bergen ${ }^{1,2}$, Guy Helman ${ }^{1}$, Cas Simons ${ }^{1}$, Neal Bennett ${ }^{19}$, Ken Nakamura ${ }^{19}$, Daniella H. Hock ${ }^{20}$, David A. Stroud ${ }^{20}$, Luke E. Formosa ${ }^{21}$, Michael T. Ryan ${ }^{21}$, Zafer Yueksel ${ }^{22}$, Beryl B. Cummings ${ }^{23}$, Hakon Hakonarson ${ }^{24}$, David R. Thorburn ${ }^{1,2}$ and John Christodoulou ${ }^{1,2}$

${ }^{1}$ Murdoch Children's Research Institute, Melbourne, Australia, ${ }^{2}$ Department of Paediatrics, University of Melbourne, Melbourne, Australia, ${ }^{3}$ Rare Diseases Functional Genomics, The Children's Hospital at Westmead, Sydney, Australia, ${ }^{4}$ Discipline of Child \& Adolescent Health, University of Sydney, Sydney, Australia, ${ }^{5}$ Kinghorn Centre for Clinical Genomics, Garvan Institute of Medical Research, Sydney, Australia, ${ }^{6}$ Children's Cancer Institute \& School of Women's and Children's Health, University of New South Wales, Sydney, Australia, ${ }^{7}$ Genetic Metabolic Disorders Service, The Children's Hospital at Westmead, Sydney, Australia, ${ }^{8}$ Department of Metabolic Medicine, Queensland Children's Hospital, Brisbane, Australia, ${ }^{9} \mathrm{~S} c h o o l$ of Clinical Medicine, University of Queensland, Brisbane, Australia, ${ }^{10}$ School of Medicine, Griffith University, Gold Coast, Australia, ${ }^{11}$ Genetic Metabolic Disorders Service, The Children's Hospital at Westmead, Sydney, Australia, ${ }^{12}$ Discipline of Genetic Medicine, Sydney Medical School, University of Sydney, Sydney, Australia, ${ }^{13}$ Hunter Genetics, Newcastle, Australia, ${ }^{14}$ Metabolic Unit, Department of Rheumatology and Metabolic Medicine, Perth Children's Hospital, Perth, Australia,

${ }^{15}$ Department of Neurology, Princess Margaret Hospital for Children/Perth Children's Hospital, Perth, Australia, ${ }^{16}$ School of Paediatrics and Child Health, University of Western Australia, Perth, Australia, ${ }^{17}$ Department of Clinical Genetics, Sydney Children's Hospital Randwick, Sydney, Australia, ${ }^{18}$ Department of Neurogenetics, Kolling Institute of Medical Research, University of Sydney and Royal North Shore Hospital, Sydney, Australia, ${ }^{19} \mathrm{Gladstone}$ Institute of Neurological Disease, San Francisco, USA,

${ }^{20}$ Department of Biochemistry and Molecular Biology, Bio21 Molecular Science and Biotechnology Institute, University of Melbourne, Melbourne, Australia, ${ }^{21}$ Department of Biochemistry and Molecular Biology, Monash Biomedicine Discovery Institute, Monash University, Melbourne, Australia, ${ }^{22}$ Bioscientia Center for Human Genetics, Ingelheim, Germany, ${ }^{23}$ Broad Institute of Harvard and Massachusetts Institute of Technology, Cambridge, USA and ${ }^{24}$ Center for Applied Genomics, Children's Hospital of Philadelphia, Philadelphia, USA

Background: The molecular diagnosis of patients with childhood onset mitochondrial disease can be challenging and may require invasive diagnostic techniques. Mitochondrial diseases can have any mode of inheritance; the most common pattern in children is autosomal recessive. Genome sequencing (GS) has the advantage of effectively analyzing both mitochondrial and nuclear genes, including coding and non-coding regions. Sequencing family trios enables phasing of variants which is particularly advantageous when an autosomal recessive pattern of inheritance is suspected. Methods: We performed genome sequencing of DNA extracted from blood of 40 pediatric patients with suspected mitochondrial disease and their parents. The sequencing data were interrogated to evaluate the diagnostic utility of trio GS, and where needed, further functional studies were undertaken. Results: To date, $58 \%$ of the patients in the cohort have reached a molecular diagnosis. Pathogenic variants were identified in 22 different genes, $83 \%$ encoded in the nuclear genome, and $17 \%$ in the mtDNA. Cases from this cohort that highlight the utility of trio GS include the identification of variants in known and novel disease genes, a deep intronic variant, and an apparently synonymous variant that creates a splicing defect. Interrogation of trio 
GS data also allowed analysis of mtDNA inheritance, showing an absence of biparental mitochondrial DNA transmission. Conclusion: Trio GS was useful in identifying variants in both nuclear and mitochondrial genes. Variant prioritization was facilitated by simultaneous segregation. The diagnostic utility was increased by combining trio GS with other molecular techniques such as RNA and cDNA studies.

\section{Loeys-Dietz Syndrome Type 2: A Rare Case}

Prashant Kumar Verma ${ }^{1}$, Henuka Verma ${ }^{1}$, Nikhil Rajvanshi ${ }^{1}$, Vyas Kumar Rathaur ${ }^{1}$, Raksha Ranjan ${ }^{1}$, Niladri Sekhar Bhunia ${ }^{1}$ and Swathi Chacham ${ }^{1}$

${ }^{1}$ All India Institute of Medical Sciences, Rishikesh, India

Hemifacial microsomia is defined as an asymmetrical congenital malformation of tissues developed from first and second branchial arches with or without multisystem involvement. Alternatively recognized as a Goldenhar syndrome or oculoauriculovertebral spectrum (OAVS); etiologically is a heterogeneous group of disorder showing dominant trends inheritable form. Loeys-Dietz syndrome is characterized by aortic root enlargement or type A dissection with or without other vascular malformations and facial midline defects. Molecular testing is essential for establishing the diagnosis because of the overlapping features of other connective tissue disorders. We are presenting a case with coinciding craniofacial features of craniofacial microsomia and Loeys-Dietz syndrome. He has a unilateral hypoplastic half face, asymmetrical ear malformations, multiple preauricular tags with epibulbar dermoid (Goldenhar syndrome); on further evaluation, he was found to have positive Brighton criteria with arterial tortuosity. Molecular testing confirmed heterozygous mutation in the TGFBR2 $(+)$ gene on intron 7 confirming the diagnosis of Loeys-Dietz syndrome type 2 . The patient is under follow-up and doing well.

\section{Improving the Yield of Trio Whole Exome Sequencing by Considering Genes with Incomplete Penetrance and Variable Expressivity}

Shuxiang Goh ${ }^{1}$, Samantha Sundercombe ${ }^{2}$, Janice Fletcher ${ }^{3}$ and Michael Buckley ${ }^{2}$

${ }^{1}$ Liverpool Hospital, Sydney, Australia, ${ }^{2}$ SEALS, Sydney, Australia and

${ }^{3}$ SA Pathology, Adelaide, Australia

Recent years have seen an improvement in the yield of trio whole exome sequencing (WES). However, a substantial portion still have negative results. One reason for a negative result is that the pathogenic variant is present in the sequencing data, but has been discarded because it has been mistaken as a benign familial variant. Method: An extensive literature review involving Pubmed and/or OMIM searches for nearly 5000 genes in the known Mendeliome for evidence of incomplete penetrance or variable expressivity. Results: Excluding genes that are inherited in a standard autosomal recessive manner, 520 other genes with published evidence of an inheritance mechanism such that an unaffected parent can pass a pathogenic variant to an affected child were identified. This list of genes forms our RedPen Database. Although the genes in this list are mostly included due to evidence for incomplete penetrance and variable expressivity, some exhibit rarer forms of inheritance, including imprinting and digenic inheritance. In addition, there are about two dozen rarer mechanisms of inheritance where a pathogenic variant can be passed from an apparently unaffected parent to an affected child. Analyzing variants in these genes after a negative trio WES, has led to identification of pathogenic or likely pathogenic variants in probands, that were inherited from their apparently unaffected parent. Conclusion: Analysis of our RedPen genes in the context of a negative exome has the potential for a modest increase in yield for trio WES.

\section{Autosomal Dominant Retinal Dystrophy due to C.1430A $>$ G in Retinoid Isomerohydrolase (RPE65)}

Rachael Heath Jeffery ${ }^{1}$, Juanita Pappalardo ${ }^{1}$, Jennifer Thompson ${ }^{2}$, Enid Chelva ${ }^{2}$, Quang Pham ${ }^{1}$, Ian Constable ${ }^{1}$, Terri McLaren ${ }^{2}$, Tina Lamey ${ }^{2}$, John De Roach ${ }^{2}$ and Fred Chen ${ }^{1}$

${ }^{1}$ Lions Eye Institute, Perth, Australia and ${ }^{2}$ Australian Inherited Retinal Disease Registry and DNA Bank, Department of Medical Technology and Physics, Sir Charles Gairdner Hospital, Perth, Australia

Background: The first TGA approved in vivo gene therapy in Australia is Luxturna (Novartis) which aims to restore retinoid isomerohydrolase (RPE65, OMIM: \#180069) function. Although recessive mutations in RPE65 are rare, the dominant RPE65-associated retinal dystrophy due to the c.1430A $>\mathrm{G}$ (Asp477Gly) variant is gaining recognition as a more common manifestation of RPE65-related retinal disease. Aim: We report novel features on multimodal retinal imaging and the natural history of a family possessing this variant in combination with a BEST1 variant. Methods: Members of a family with RPE65.1430A $>$ G were examined clinically to ascertain phenotype and underwent genetic testing. Multimodal ophthalmic imaging included microperimetry, widefield colour fundus photography, quantitative autofluorescence (qAF), spectral domain optical coherence tomography and electrophysiology. Results: The RPE65 mutation was confirmed as the causative variant and an additional likely pathogenic mutation in BEST1, c.37C $>\mathrm{T}$, was also identified. Vision loss in the proband was attributed to foveal atrophy while choroidal neovascularization and vitello-eruptive lesions led to impaired vision in one affected son. Microperimetry was able to demonstrate disease progression. Peripheral subretinal white deposits were seen in three patients and may represent genetic defects of the retinoid cycle. The mean qAF values in the proband (age 79) and the middle son (age 60) were comparable to a 20-year-old also indicating impaired retinoid recycling and reduced lipofuscin. Conclusions: The coexistence of the pathogenic BEST1 c.37C > T variant may modify clinical features observed in RPE65-associated retinal dystrophy. A lifetime of impaired retinoid isomerohydrolase function due to the Asp477Gly leads to reduced lipofuscin in the retina.

\section{Genetic Diagnosis of Acute Myeloid Leukemia by Next Generation Sequencing (NGS)}

Siew Ling (Grace) Mok ${ }^{1}$

${ }^{1}$ Royal College of Pathologists of Australasia Quality Assurance Program, Sydney, Australia

Next-generation sequencing (NGS) has redefined the genetic landscape of acute myeloid leukemia (AML), providing new molecular markers for diagnostic and prognostic classifications. To evaluate the proficiencies of laboratories utilizing Next Generation Sequencing (NGS) to detect acute myeloid leukemia (AML) in cancer-related genes, an external quality assessment (EQA) was implemented by the Royal College of Pathologists of Australasia (RCPAQAP) in 2018. In 2018, a panel of samples that comprised of 24 genes was identified as harbouring DNA variants, with 15 of these gene variants being detected by three or more laboratories (>38\%). In 2019, nine laboratories enrolled using patient derived samples and multiple target genes and regions were tested. Each 
laboratory received two purified DNA samples for analysis. Laboratory proficiency was assessed using consensus data. The results were judged to be either concordant; discordant or not assessed. Six laboratories (67\%) achieved full concordance in both samples for all consensus variants in the sample. Of the 10 gene variants, 8 genes comprised of detection of rate between $86 \%-100 \%$. Only the ZRSR2 gene gave a detection rate of $17 \%$ among the participating laboratories. There was a significant improvement in the rate of detection in 2019 compared to 2018. This data identifies the laboratories are performing at high level for detection of DNA variants in Acute myeloid leukemia. As part of our EQA of laboratories performing targeted NGS, detailed analyses of the results were provided so that all participants became aware of the performance of various workflows and the methodologies utilized.

\section{MAGEL2 Imprinting Variants}

Louisa Sanchez ${ }^{1}$, Evelyn Douglas ${ }^{1}$, Jessica Burdett ${ }^{1}$, Shannon Le Blanc ${ }^{2}$, Nicholas Smith ${ }^{2}$, Andrew Dubowsky ${ }^{1}$, Kathie Friend ${ }^{1}$ and Sui $\mathrm{Yu}^{1}$

${ }^{1}$ SA Pathology, Adelaide, South Australia and 'Women's and Children's Hospital, Adelaide, South Australia

Pathogenic MAGEL2 variants result in the phenotypes of ChitayatHall (CHS), Schaaf-Yang (SYS) and Prader-Willi (PWS) syndromes. Schaaf-Yang Syndrome is characterized by neonatal hypotonia, developmental delay, intellectual disability, feeding problems in infancy, joint contractures and autism spectrum disorder, sharing clinical overlap with Prader-Willi Syndrome and Chitayat-Hall syndrome. SYS has been associated with truncating variants in MAGEL2, a maternally imprinted gene. A neuromuscular gene panel analysis was requested on a two week old male infant, requiring respiratory support, with hypotonia, bilateral talipes and central apneas. No variants explaining the patient's phenotype were initially identified by this analysis, but subsequent reanalysis of the exome revealed a truncating variant in the MAGEL2 gene. Parental studies confirmed this variant to be paternally inherited, consistent with the inheritance pattern of Schaaf-Yang Syndrome (imprinting). Following this finding, the MAGEL2 gene was added to our standard neuromuscular panel. To date, only one additional variant has been identified in MAGEL2, an in-frame deletion of 30 nucleotides. Parental studies indicated this variant to be maternally inherited, so it is considered unlikely to be disease causing.

\section{Mosaicism in Next-Generation Sequencing Analysis - A Tale of Caution}

Evelyn Douglas ${ }^{1}$, Louisa Sanchez ${ }^{1}$, David Lawrence ${ }^{2}$, Julien Soubrier ${ }^{1}$, Song Gao ${ }^{1}$, Lesley McGregor ${ }^{3}$, Christopher Barnett ${ }^{3}$, Karin Kassahn ${ }^{1}$, Sui Yu ${ }^{1}$ and Kathie Friend ${ }^{1}$

${ }^{1}$ Genetics and Molecular Pathology, SA Pathology, Adelaide, Australia, ${ }^{2}$ ACRF Cancer Genomics Facility, Adelaide, Australia and ${ }^{3}$ Paediatric and

Reproductive Genetics, SA Clinical Genetics Service, Adelaide, Australia

The occurrence of mosaicism, the presence of two or more populations of cells with distinct genotypes in an individual, needs consideration when analyzing next-generation sequencing data. It has been reported that $0.5-8.3 \%$ of apparently de novo pathogenic variants in diseases with autosomal dominant inheritance have a parent who is a low level mosaic (variant allele frequency (VAF) of 2-29\%) for that variant. This has implications for future pregnancies (recurrence risk). Automated trio analysis pipelines using an autosomal dominant de novo analysis may discard these variants depending on their parameters. A separate phenotype analysis comparing VAF in the apparently unaffected parents is one way of highlighting these inherited 'de novo' variants. Using this approach, we have detected a mosaic TGFBR2 variant (VAF of 20\%) in a clinically unaffected individual with multiple children with Marfan syndrome and identified a heterozygous HECW2 variant in a patient with intellectual disability and severe autistic features. His clinically unaffected father was mosaic for this change (VAF of 30\%).Additionally, if somatic mosacisim is suspected in cases where constitutional pathogenic variants are undetected, then analysis should be undertaken on the appropriate tissue type. Next generatioin sequencing analysis of an appropriate skin biopsy tissue in a child with a clinical diagnosis of Klippel-Trenaunay-Weber syndrome revealed a variant in the PIK3CA gene (VAF 12.7\%). The variant may not be present at a detectable level in a different tissue such as whole blood. A discussion of our mosaic findings will be presented.

\section{Reproductive Carrier Screening in an Australian Private Pathology Setting}

Elise Francis ${ }^{1}$, Chloe La Spina ${ }^{1}$, Terri Sivertsen ${ }^{1}$ and Melanie Galea ${ }^{1}$

${ }^{1}$ Douglass Hanly Moir Pathology, Australia

The Royal Australian and New Zealand College of Obstetricians and Gynaecologists (RANZCOG) and Human Genetics Society of Australasia (HGSA) now recommend that information regarding reproductive carrier screening for common monogenic genetic conditions be available to all women planning pregnancy or in their first trimester. We are a private clinical pathology laboratory, servicing an Australia-wide pathology network. Here we present a retrospective audit of 3 years of offering a 3-gene reproductive carrier screen in this private pathology setting. The conditions covered by this screen are cystic fibrosis (CF), fragile X syndrome (FXS) and spinal muscular atrophy (SMA). This is an optional test, available to general practitioners and specialist clinicians. Therefore, this study aimed to identify the current trends of reproductive carrier screening in Australia and to examine CF, SMA and FXS carrier frequency in the examined cohort. The demographics examined include patient gender, age, and pregnancy status and referrer trends. Furthermore, the proportion of patients with a known family history of one of these conditions was investigated. This study highlights the importance and relevance of reproductive carrier screening in all couples. It is evident that earlier screening allows high risk couples to become informed and in turn make valuable reproduction decisions.

\section{Novel Variant Investigation in Patient-Derived Retinal Organoids to Increase Genetic Diagnosis in the Retinal Dystrophies}

Fidelle Chahine Karam ${ }^{1}$, To Ha Loi ${ }^{1}$, Alan Ma ${ }^{1,2,4}$, Benjamin Nash ${ }^{1,3,4}$, Bruce Bennetts ${ }^{3,4}$, Anai Gonzalez-Cordero ${ }^{5}$ and Robyn Jamieson ${ }^{1,2,4,6}$

${ }^{1}$ Eye Genetics Research Unit, Children's Medical Research Institute, University of Sydney, Sydney, Australia, ${ }^{2}$ Department of Clinical Genetics, Western Sydney Genetics Program, The Children's Hospital at Westmead, Sydney Children's Hospitals Network, Sydney, Australia, ${ }^{3}$ Sydney Genome Diagnostics, Western Sydney Genetics Program, The Children's Hospital at Westmead, Sydney Children's Hospitals Network, Sydney, Australia, ${ }^{4}$ Specialty of Genomic Medicine, Faculty of Medicine and Health, University of Sydney, Sydney, Australia, ${ }^{5}$ Stem Cell Medicine Group, Children's Medical Research Institute, University of Sydney, Sydney, Australia and 'Save Sight Institute, University of Sydney, Sydney, Australia

Background: There is marked genetic heterogeneity in the retinal dystrophies, with additional novel variants detected during 
diagnostic testing. Classifying pathogenicity of novel variants provides genetic information and allows access to clinical trials and new therapies. We use patient-derived induced pluripotent stem cells (iPSCs), differentiated to retinal pigment epithelium (iPSC-RPE) and retinal organoids (iPSC-RO), to determine pathogenicity and as a platform to test novel therapies. Hypothesis: iPSC-RPE and iPSC-RO can be used to investigate pathogenicity of a novel intronic RPGR variant in an X-linked family. Methods: Patient fibroblasts were used to create iPSC clonal cell lines, which were differentiated to retinal cells. In this study, an RPGR intron 11 variant was reported diagnostically as a variant of uncertain significance. cDNA sequencing was used to investigate RNA splicing. RPGR expression studies used qRTPCR and immunofluorescence in iPSC-RPE and ROs. RPGR protein interactions and downstream functions were explored through co-immunofluorescence in iPSC-ROs. Results: The novel RPGR variant demonstrated aberrant splicing. Gene expression of RPGR in patient iPSC-RPE and iPSC-ROs was decreased. RPGR protein immunofluorescence in patient primary cilia revealed decreased expression and mislocalization. Loss of RPGR protein complexes and cytoskeletal dysregulation in patient-derived iPSC-ROs was demonstrated. Conclusion: Our approach using iPSC-RPE and ROs revealed the novel intronic RPGR variant resulted in abnormal splicing, decreased expression, abnormal cilia and impaired RPGR function. This facilitated variant classification to likely pathogenic, provided genetic information and eligibility for future clinical trials for affected family members, indicating the value of this approach in the retinal dystrophies.

\section{An Incidental Finding from Genetic Investigations for Global Developmental Delay}

Chloe La Spina ${ }^{1}$, Dallas Grasby ${ }^{1}$, Elise Francis ${ }^{1}$, Terri Sivertsen ${ }^{1}$, Eric Lee ${ }^{1,2}$ and Melanie Galea ${ }^{1}$

${ }^{1}$ Douglass Hanly Moir Pathology, Sydney, Australia and ${ }^{2}$ Sullivan Nicolaides Pathology, Australia

Incidental findings are defined as abnormal results that are identified unexpectedly though genetic analysis, which were unrelated to the initial testing purpose. Here, we present a case study that began with the investigation of a child with global developmental delay (GDD), and through multiple molecular testing techniques led to an incidental finding. As recommended by the American College of Medical Genetics and Genomics, first tier testing for GDD included the investigation of fragile $\mathrm{X}$ syndrome and chromosomal microarray analysis. Microarray detected a novel 14q13.1 duplication, initially classified as a variant of uncertain significance, and an unexpected finding of a 16p13.3 deletion, containing the HBA gene cluster. To further interrogate the pathogenicity and inheritance pattern of this result, parental microarray testing was conducted. In addition, given the limited resolution of microarray at the HBA gene cluster, follow-up testing was carried out using targeted multiplex ligation-dependant probe amplification (MLPA) for the trio, to define the extent and zygosity of the deletion. The 14q13.1 duplication was inherited from the proband's asymptomatic mother, which provided evidence in favour of a likely benign classification. The MLPA results were consistent with a paternally inherited heterozygous -SEA deletion. In summary, the cause of GDD in this proband remains unknown and further testing, such as exome analysis by massively parallel sequencing, may be considered. Investigations involving the HBA gene cluster were able to characterize the -SEA deletion in the proband and his father. This incidental finding could be used to guide future reproductive decisions.

\section{Functional Genomics Capacity Within Diagnostic Laboratories}

Tessa Mattiske ${ }^{1,2}$, Nigel Laing ${ }^{6,7}$, Jozef Gecz ${ }^{9,10,11}$, Bruce Bennett ${ }^{18}$, Kristi Jones ${ }^{12}$, Robert Bryson Richardson ${ }^{8}$, Kelly Smith ${ }^{13}$, Ian Smyth ${ }^{15}$, Patrick Tam ${ }^{16,17}$, Zornitza Stark ${ }^{3,14}$, Sally Dunwoodie ${ }^{4,5}$ and Andrew Sinclair ${ }^{1,2,3}$

${ }^{1}$ Australian Genomics, Australia, ${ }^{2}$ Murdoch Children's Research

Institute, Melbourne, Australia, ${ }^{3}$ University of Melbourne, Melbourne, Australia, ${ }^{4}$ Victor Chang Cardiac Research Institute, Sydney, Australia, ${ }^{5}$ University of New South Wales, Sydney, Australia, ${ }^{6}$ Centre for Medical Research, The University of Western Australia, Perth, Australia, ${ }^{7}$ Harry Perkins Institute for Medical Research, Perth, Australia, ${ }^{8}$ School of Biological Sciences, Monash University, Melbourne, Australia, ${ }^{9}$ Adelaide Medical School and the Robinson Research Institute, The University of Adelaide, Adelaide, Australia, ${ }^{10}$ Women and Kids, South Australian Health and Medical Research Institute, Adelaide, Australia, ${ }^{11}$ Genetics and Molecular Pathology, SA Pathology, Adelaide, Australia, ${ }^{12}$ Kids Neuroscience, The Children's Hospital at Westmead, Sydney, Australia, ${ }^{13}$ Department of Physiology, University of Melbourne, Melbourne, Australia, ${ }^{14}$ Victorian Clinical Genetics Services, Murdoch Children's Research Institute, Melbourne, Australia, ${ }^{15}$ Department of Anatomy and Developmental Biology, Development and Stem Cells Program, Monash Biomedicine Discovery Institute, Monash University, Melbourne, Australia, ${ }^{16}$ Embryology Unit, Children's Medical Research Institute, University of Sydney, Sydney, Australia, ${ }^{17}$ School of Medical Sciences, Faculty of Medicine and Health, University of Sydney, Sydney, Australia and ${ }^{18}$ Department of Molecular Genetics, The Children's Hospital at Westmead, Sydney, Australia

Functional genomics ( $\mathrm{FxGx})$ is the testing of a gene at the DNA, RNA and/or protein level to study the effect of variant/s on normal function. It is considered to provide strong evidence relating to the clinical significance of an individual variant. The Australian Functional Genomics Network (AGFN) surveyed Australian accredited genetic testing laboratories to determine the pathways in which they are seeking out FxGx testing for variants detected in patients and how FxGx evidence is being incorporated into variant classifications. Responses to date have been collected from 10 laboratories. All indicated FxGx data would be helpful to clarify pathogenicity of variants. However, $30 \%$ do not follow up with further investigations. $70 \%$ of laboratories do follow up with mRNA assays and $60 \%$ with other FxGx testing. Not knowing who to contact (25\%), cost (25\%), and time (17\%) are the top barriers to accessing FxGx testing. As demand grows for FxGx testing, $80 \%$ of laboratories stated they would prefer accessing tests through specialized research laboratories with some form of accreditation in additional to $67 \%$ laboratories indicating to wanting to develop in-house tests. All laboratories reported determining the quality of data was the biggest hurdle when incorporating FxGx data in classification scoring. These results highlight the need to recognize FxGx testing as an essential diagnostic testing procedure, the need to develop standard FxGx reporting guidelines and dedicated funding for FxGx testing. The AFGN continue to build a registry of research laboratories performing FxGx testing to bridge the gap between diagnostic teams and researchers. 


\section{Neurogenetics: Variation in THOC2 MRNA Export Factor Affects TREX Complex Protein Stability and Causes Variable Neurodevelopmental Disturbance}

Raman Sharma ${ }^{1}$, Elizabeth Palmer ${ }^{2,3}$, Alison Gardner ${ }^{1}$, Renee Carroll ${ }^{1}$, Danielle Mazurkiewicz ${ }^{1}$, Rudrarup Bhattacharjee ${ }^{1}$, Luis Perez-Jurado ${ }^{4,5}$, Melanie Leffler ${ }^{2}$, Michael Field ${ }^{2}$ and Jozef Gecz ${ }^{1}$

${ }^{1}$ Adelaide Medical School, The University of Adelaide and The Robinson Research Institute, Adelaide, Australia, ${ }^{2}$ GOLD Service, Hunter Genetics, Newcastle, Australia, ${ }^{3}$ School of Women's and Children's Health, UNSW Sydney, Adelaide, Australia, ${ }^{4}$ South Australian Clinical Genetics Service, SA Pathology, Adelaide, Australia and ${ }^{5}$ Hospital del Mar Research Institute, Network Research Centre for Rare Diseases and Universitat Pompeu Fabra, Barcelona, Spain

Highly conserved TREX (Transcription-Export)-mediated mRNA export is emerging as a key pathway in neuronal development and differentiation. TREX subunit variants cause neurodevelopmental disorders (NDDs) by interfering with mRNA export from the cell nucleus to the cytoplasm. We have so far identified twenty missense, three splicing-defective (e.g., Exon35:c.4450-2A>G) and one deletion THOC2 (e.g., $\triangle \mathrm{Ex} 37-38$ ) variant. Our data from ex vivo missense variant testing and patient-derived cell lines showed 9 of the 14 missense variants result in reduced protein stability (e.g., p.Leu438Pro), and all of splicing-defective and deletion variants lead to loss of small regions of C-terminal THOC2 RNA binding domain. A number of other TREX subunits (e.g., THOC1, THOC4, THOC5, THOC7, THOC6) have also been associated with NDDs (unpublished data). Interestingly, reduced stability of THOC2 variant proteins also affects stability of other NDD-associated THOC subunits. Along with the core intellectual disability (ID) phenotype, additional features include persistent hypotonia, pre- and postnatal growth restriction, brain malformations, epilepsy and other congenital anomalies. This demonstrates that the phenotypic spectrum is much broader than non-syndromic ID. While elucidating precisely how TREX subunit variants cause NDDs continues to be a challenge, the current evidence suggests that alterations to the canonical mRNA export pathway, otherwise essential for cellular life, can be compatible with life but lead to a range of developmental disabilities, neurodegeneration and cancer in humans.

\section{Severe Early Infantile Epileptic Encephalopathy Caused by a KCNT1 Pathogenic Variant Inherited From a Mildly Affected Parent: A Cautionary Tale}

\footnotetext{
Sarah Heron ${ }^{1}$, Sebastian Lunke ${ }^{2,3,4}$, Alison Gardner ${ }^{1}$, Sophie Bouffler,5, Clair Pridmore ${ }^{6}$, Christopher Barnett ${ }^{6}$, Zornitza Stark ${ }^{2,4,5}$ and Jozef Gécz ${ }^{1,7}$

${ }^{1}$ University of Adelaide, Adelaide, Australia, ${ }^{2}$ Victorian Clinical Genetics Services, Melbourne, Australia, ${ }^{3}$ Murdoch Children's Research Institute, Melbourne, Australia, ${ }^{4}$ The University of Melbourne, Melbourne, Australia, ${ }^{5}$ Australian Genomics, Melbourne, Australia, ${ }^{6}$ Women's and Children's Hospital, Adelaide, Australia and ${ }^{7}$ South Australian Health and Medical Research Institute, Adelaide, Australia
}

Background: Heterozygous pathogenic variants in KCNT1 most frequently cause epilepsy of infancy with migrating focal seizures (EIMFS) or a severe form of nocturnal frontal lobe epilepsy (NFLE). Patients with EIMFS generally have de novo variants while NFLE cases can be de novo or familial. Both phenotypes occasionally occur within the same family. Unaffected individuals carrying pathogenic KCNT1 variants are rare, and most have variants associated with the milder NFLE phenotype. Research Question: We identified an apparently heterozygous mother of a patient with early infantile epileptic encephalopathy caused by a KCNT1 pathogenic variant, c.862G $>$ A; p.Gly288Ser. The variant was excluded during the original trio analysis due to it being inherited. Further assessment showed that it had been reported in 19 unrelated patients with epileptic encephalopathies or severe NFLE. We sought to determine whether the mild maternal phenotype could result from somatic mosaicism. Methods: Maternal DNA extracted from blood, hair root, buccal swab and saliva was analyzed for the variant using digital droplet PCR. Heterozygous patient DNA mixed with control DNA at varying ratios was analyzed to confirm accurate quantitation of mosaicism. Results: Unexpectedly, there was no evidence of mosaicism in the mother. Examination of medical records showed that she had borderline intellectual disability and had had a single febrile seizure. Conclusions: An established, recurrent pathogenic KCNT1 variant leading to EIMFS was detected constitutively in a mildly affected individual. This has not been observed previously and suggests that extreme variability in clinical expressivity, even of known pathogenic variants, occurs in KCNT1-related disorders.

\section{Assessing Neurocognitive Function in a Naturally Occurring Murine Model of Mucopolysaccharidosis Type IIIA}

Kleopatra Pericleous $^{1,2}$, Chantelle McIntyre ${ }^{1,2}$ and Maria Fuller ${ }^{1,2}$

${ }^{1}$ The University of Adelaide, Adelaide, Australia and ${ }^{2}$ SA Pathology, Adelaide, Australia

Mucopolysaccharidosis type IIIA (MPS IIIA) is an autosomal recessive lysosomal storage disorder that manifests a progressive neurological phenotype characterized by autistic-like behaviors and a loss of acquired skills. Disease onset is typically in early childhood and there is no clinically approved treatment for the relentless neurological decline. A naturally occurring MPS IIIA mouse model is being extensively utilized to investigate potential treatments but a major challenge is the ability to accurately and effectively assess whether tested treatments improve neurological function. To address this, the neuropsychiatric and neurocognitive performance of the MPS IIIA mouse model was assessed by subjecting the mice to a battery of rodent tests at different stages of disease progression. Sample size ranged from 7-11 mice per genotype per group. The water cross-maze showed that learning and memory was impaired in MPS IIIA mice at 6 and 8 months of age. Hypo-activity was observed in the open field at 6 and 9 months of age in MPS IIIA mice and gait assessment revealed impaired locomotion at 9 months. Reduced motivation and well-being was observed at 9 months through burrowing assessment and at 6 and 10 months in nest building assessments in the IIIA mice. Finally, seed opening test implied diminished fine motor skills in the MPS IIIA mice at 10 months of age. Mice tested at 3 months were comparable to the wild-type counterparts in all assessments. This study provides insight into the efficacy and reliability of behavioral testing in the MPS IIIA mouse model.

\section{Comprehensive Exonic Sequencing of Hemiplegic Migraine Related Genes in a Cohort of Probands Identifies Known and Potential Pathogenic Variants}

Heidi Sutherland ${ }^{1}$, Neven Maksemous ${ }^{1}$, Cassie Albury ${ }^{1}$, Omar Ibrahim $^{1}$, Robert Smith ${ }^{1}$, Rod Lea ${ }^{1}$, Larisa Haupt ${ }^{1}$, Bronwyn Jenkins ${ }^{2}$, Benjamin Tsang ${ }^{3}$ and Lyn Griffiths ${ }^{1}$

${ }^{1}$ Queensland University of Technology, Brisbane, Australia, ${ }^{2}$ The Epping Clinic, Sydney, Australia and ${ }^{3}$ Department of Neurology, Sunshine Coast University Hospital, Birtinya, Australia 
Background: Hemiplegic migraine (HM) is a rare migraine disorder with aura subtype including temporary weakness and visual, sensory and/or speech symptoms. To date three main genes - CACNA1A, $A T P 1 A 2$, and SCN1A - have been found to cause HM. These encode ion channels or transporters, important for regulating neuronal ion balance and synaptic transmission, leading to HM being described as a channelopathy. However, $<20 \%$ of HM cases referred for genetic testing have mutations in these genes and other genes with roles in ion and solute transport, and neurotransmission have also been implicated in some HM cases. Aim: To investigate pathogenic variants in other genes that have been previously linked to $\mathrm{HM}$, or overlapping conditions, in HM cases who remain without a molecular diagnosis. Methods: We performed whole exome sequencing for 187 suspected HM probands referred for genetic testing, but found to be negative for CACNA1A, ATP1A2 and SCN1A mutations, and applied targeted analysis of whole exome sequencing data for rare missense or potential protein-altering variants in the $P R R T 2$, PNKD, SLC1A3, SLC2A1, SLC4A4, ATP1A3 and ATP1A4 genes. Results: Known mutations and some potentially pathogenic variants were identified in each of these genes in specific cases, for up to $10 \%$ of the cohort. Discussion/Conclusion: Screening of these additional HM-related genes in HM patients referred for genetic testing improves molecular diagnosis for the disorder. However, twothirds were found not to have candidate mutations in any of the previously reported HM genes, suggesting that additional genetic factors contributing to the disorder are yet to be identified.

\section{Mitochondrial Variation From Whole Exome Sequencing Identifies New Candidate Mutations in Cerebrovascular Disorders}

Paul Dunn ${ }^{1}$, Neven Maksemous ${ }^{1}$, Robert Smith ${ }^{1}$, Heidi Sutherland ${ }^{1}$, Larisa Haupt ${ }^{1}$ and Lyn Griffiths ${ }^{1}$

${ }^{1}$ Queensland University of Technology (QUT), Centre for Genomics and Personalised Health, Genomics Research Centre, School of Biomedical Sciences, Institute of Health and Biomedical Innovation, Brisbane, Australia

Background: Cerebral autosomal dominant arteriopathy with subcortical infarcts and leucoencephalopthy (CADASIL) is a cerebral small vessel disease and the most common monogenic form of inherited stroke and vascular dementia. A common differential diagnosis in CADASIL patients is related to mitochondrial encephalopathy with lactic acidosis and stroke-like episodes (MELAS) or mitochondrial encephalopathy with ragged red fibers (MERFF). The aim of this project was to identify mutations affecting the mitochondria within mitochondrial DNA (mtDNA) and nuclear encoded mitochondrial proteins (NEMPs). Methods: Whole Exome Sequencing was performed using $n=50$ samples sent for CADASIL diagnosis using the AmpliSeq Exome RDYTM library. Extraction of the mitochondrial sequence was completed by re-aligning the bam files to the Mitochondrial Cambridge reference sequence and extracting the NEMPs from the annotated vcf files. Analysis of potential pathogenic variants was completed using in silico pathogenicity prediction tools and population databases where candidate mutations were identified if $<2$ tools predicted a benign/tolerated reading with a MAF of $<0.001$. Results: We confirmed the missense mutations previously identified in MELAS genes (MTND5 $\neg \mathrm{m} .13468 \mathrm{C}>\mathrm{A}$ and POLG NM_001126131.2:c.2209G $>$ C). A further three variants (m.6160C $>$ T, m.6716A $>$ G and m.15058C $>$ T) were identified across 4 samples (DGR322, DGR343, DGR020 and DGR350 respectively) as candidate pathogenic mutations in the extracted mitochondrial genome. Conclusion: Analysis of NEMP and mtDNA variants extracted from whole exome sequencing data can aid in diagnosing the cause of inherited cerebrovascular diseases.

\section{Biallelic Loss-of-Function NRROS Variants Impairing Active TGF-B1 Delivery Cause a Severe Infantile Onset Neurodegenerative Condition With Intracranial Calcification}

Xiaomin Dong ${ }^{1,2}$, Natalie Tan ${ }^{1,2,3}$, Katherine Howell ${ }^{1,2,4}$, Sabina Barresi ${ }^{5}$,

Jeremy Freeman ${ }^{1,4}$, Davide Vecchio ${ }^{5}$, Maria Piccione ${ }^{6}$, Francesca Clementina Radio ${ }^{5}$ Daniel Calame ${ }^{7,8}$, Shan Zong ${ }^{1}$, Stefanie Eggers ${ }^{1,3}$, Ingrid Scheffer ${ }^{1,2,4,9}$, Tiong Y. Tan ${ }^{1,2,3}$, Nicole J. Van Bergen ${ }^{1,2}$, Marco Tartaglia ${ }^{5}$, John Christodoulou ${ }^{1,2,3}$ and Susan M. White Wh $^{1,2,3}$

${ }^{1}$ Victorian Clinical Genetics Services, Melbourne, Australia, ${ }^{2}$ Murdoch Children's Research Institute, Melbourne, Australia, ${ }^{3}$ Department of Paediatrics, University of Melbourne, Melbourne, Australia, ${ }^{4}$ Department of Neurology, Royal Children's Hospital, Melbourne, Australia, ${ }^{5}$ Genetics and Rare Diseases Research Division, Ospedale Pediatrico Bambino Gesù, Italy, ${ }^{6}$ Department of Science for Health Promotion and Mother and Child Care, Università degli Studi di Palermo, Italy, ${ }^{7}$ Baylor College of Medicine, Houston, USA, ${ }^{8}$ Texas Children's Hospital, Houston, USA and ${ }^{9}$ Department of Medicine, University of Melbourne, Austin Health, Melbourne, Australia

Background: NRROS is a leucine-rich repeat-containing protein that associates with latent TGF- $\beta 1$ and anchors it on the cell surface, a requirement for activation of TGF- $\beta 1$ in macrophages and microglia. We report six individuals from four families with biallelic variants in NRROS. All affected individuals had neurodegenerative disease with refractory epilepsy, developmental regression, and reduced white matter volume with delayed myelination. Intracranial calcification was detected in all who had head CT scans (3/6). Three individuals died between two to four years of age. These features are consistent with those observed in the Nrros knockout mouse and overlap with those seen in the human condition TGF- $\beta 1$ deficiency. The diseasecausing NRROS variants involve two significant functional NRROS domains, and result in aberrant NRROS proteins with impaired ability to anchor latent TGF- $\beta 1$ on the cell surface. Methods: Confocal microscopy in HEK293T cells was used to investigate the localization of wild-type and mutant NRROS proteins. Flow cytometry was performed to analyze the influence of mutant NRROS proteins in anchoring latent TGF- $\beta 1$ to the cell surface. Results: Wild-type and mutant NRROS proteins were found to co-localize with latent TGF- $\beta 1$ intracellularly. However, flow cytometry showed that the mutant NRROS proteins failed to anchor latent TGF- $\beta 1$ on the cell surface as compared to wild-type NRROS. Moreover, wild-type NRROS rescued the defect of the disease-associated mutants in presenting latent TGF- $\beta 1$ to the cell surface. Conclusion: Our findings suggest that loss of NRROS function causes a severe childhood-onset neurodegenerative condition with features suggestive of a disordered response to inflammation.

\section{Cochlear Nerve Deficiency in SOX11-Related Coffin Siris Syndrome}

Salam Mohammadsaeed Alburaiky

Genetics Health Services New Zealand, Auckland, New Zealand

Background: The phenotypic spectrum of SOX11-related Coffin Siris syndrome (CSS) is expanding with reports of new associations. SOX11 is implicated in neurogenesis and inner ear development. Cochlear nerve deficiency, absence or hypoplasia, is commonly associated with cochlear canal stenosis but can be present in CHARGE syndrome, a condition that affects inner ear development. Case 
Presentation: We present two cases of children with de novo SOX11 likely pathogenic variants and CSS features including global developmental delay, growth deficiency and bilateral hypoplastic nails. Both had unilateral sensorineural hearing loss due to cochlear nerve deficiency confirmed on MRI imaging. Discussion: SOX11 is implicated in sensory neuronal survival and maturation. It is also highly expressed in the developing inner ear. Ablation of SOX11 in animal models resulted in reduction in sensory neurons survival and decreased axonal growth and hearing loss with grossly normal inner ears structures. This further supports a new association between SOX11 related CSS and cochlear nerve deficiency. Conclusion: We propose cochlear nerve deficiency as a new phenotypic feature of SOX11-related CSS. We recommend all patients with SOX11-related CSS to have audiology screening. MRI imaging may be indicated checking for cochlear nerve deficiency.

\section{PRENATAL DIAGNOSIS}

\section{Prenatal Diagnosis of Sex Chromosome Aneuploidy Before and After the Introduction of Noninvasive Prenatal Testing: A Population-Based Study in Victoria}

Lulu Loughry ${ }^{1,2}$, Lisa Hui ${ }^{1,2}$ and Jane Halliday ${ }^{1,2}$

${ }^{1}$ The University of Melbourne, Melbourne, Australia and ${ }^{2}$ Murdoch Children's Research Institute, Melbourne, Australia

Background: Noninvasive prenatal testing (NIPT) became available in Australia in 2012. Screening for sex chromosome aneuploidy (SCA) through NIPT has increased prenatal testing for these conditions in Victoria. Aim: To analyze the frequency of SCA detection through prenatal diagnosis before and after introduction of NIPT. Methods: All prenatal diagnostic tests performed in Victoria at $<25$ weeks gestation from 2010-19 were analyzed. The annual numbers of total and individual SCAs were used to estimate the proportion of these among all prenatal diagnostic tests and all Victorian births (not including 2019 birth data). Chi-squared trend analysis was used, with $p<.05$ considered significant. Results: During the 10 -year study period, 23,937 prenatal diagnostic tests were performed, including 391 confirmed SCAs. The most common SCA was $45 \mathrm{XO}(n=178)$, followed by $47 \mathrm{XXY}(n=113), 47 \mathrm{XXX}(n=60)$ and $47 X Y Y(n=37)$. The annual number of total SCAs as a proportion of total tests increased significantly between $2010-19\left(\chi^{2}\right.$ trend $=107.8, p<.0001)$. Since 2017, 47XXY replaced $45 \mathrm{XO}$ as the most common SCA detected on prenatal diagnosis, with almost all cases in 2019 detected by NIPT (96\%). The annual numbers of total SCAs as a proportion of annual births from 2010-18 did not show a significant increase, however a significant increase was seen for 47XXY $\left(\chi^{2}\right.$ trend $=32.5, p<.0001)$. Conclusion: The introduction of NIPT has seen a significant increase in the number of SCAs being detected via prenatal diagnosis. SCAs which would have previously gone undetected on ultrasound, such as 47XXY, are now ascertained via NIPT. This has significant implications for health services.

\section{Clinical Performance of the VCGS Percept ${ }^{\circledR}$ Cell-Free DNA Prenatal Screening Test for Aneuploidy in a General Risk Population in Australia}

Katrina Scarff ${ }^{1,2}$, Nicola Flowers ${ }^{1,2}$, Clare Love $^{1,2}$, Alison Archibald ${ }^{1,2,3}$, Olivia Giouzeppos ${ }^{1,2}$, Clare Hunt ${ }^{1,2}$, Larissa Ng ${ }^{1,2}$, Monika Janinski ${ }^{1,2}$, Martin Delatycki ${ }^{1,2}$ and Mark Pertile ${ }^{1,2,3}$

${ }^{1}$ Victorian Clinical Genetics Services, Melbourne, Australia, ${ }^{2}$ Murdoch Children's Research Institute, Melbourne, Australia and ${ }^{3}$ Department of Paediatrics, University of Melbourne, Melbourne, Australia
Background: Professional bodies recommend reporting on the performance of cfDNA screening tests, which are now routinely utilized by women with high and low-risk pregnancies. Aim: To evaluate the clinical performance of percept ${ }^{\otimes}$ cell-free DNA (cfDNA) screening for fetal aneuploidy in a general risk obstetric population in Australia and New Zealand. Method: This was a retrospective analysis of a general obstetric cohort, tested prospectively at VCGS by percept $^{\circledast}$ cfDNA prenatal screening. Whole genome sequencing of cfDNA in the maternal circulation was performed to detect fetal aneuploidy. The first 38,289 pregnancies tested were reviewed, as were other tests performed in these pregnancies. Results: Cytogenetic and other pregnancy outcomes were obtained for $91.3 \%(609 / 667)$ of high-risk singleton cases. The positive predictive values (PPVs) were $98.5 \%$ for T21, 92.5\% for T18 and $55.2 \%$ for T13. PPVs for sex chromosome aneuploidies ranged from 30-95.2\%. There were 111 cases $(0.3 \%)$ at increased-risk for a rare autosomal trisomy, $44 \%$ of which were associated with missed miscarriage. Analysis of other cytogenetic tests of the pregnancy/resulting child identified chromosome conditions not detected by cfDNA screening, including triploidy and copy number variants. 93.7\% (564/602) of women having cfDNA screening after a high-risk maternal serum screening (MSS) result received a low-risk cfDNA result, and avoided invasive diagnostic testing. Conclusions: cfDNA screening was highly accurate for detecting fetal aneuploidy in this population. Other tests performed on these pregnancies identified chromosome abnormalities not detected by cfDNA screening. Contingent cfDNA screening after high-risk MSS significantly reduced the number of invasive diagnostic procedures required.

\section{The Expanding Scope of Noninvasive Prenatal Testing (NIPT) in Victoria: A Population-Based Study of Prenatal Diagnosis After NIPT}

Lisa Hui ${ }^{1,2}$, Lulu Loughry ${ }^{1}$ and Jane Halliday ${ }^{1,3}$

${ }^{1}$ Murdoch Children's Research Institute, Melbourne, Australia, ${ }^{2}$ Department of Obstetrics and Gynaecology, University of Melbourne, Melbourne, Australia and ${ }^{3}$ Department of Paediatrics, University of Melbourne, Melbourne, Australia

Background: Over the past 5 years, noninvasive prenatal testing for fetal chromosome conditions has expanded to include conditions beyond the three common autosomal trisomies. Aim: To analyze fetal chromosome abnormalities ascertained via NIPT and to estimate the positive predictive value (PPV) of NIPT for the different conditions. Methods: A population-based analysis of all prenatal diagnostic testing $<25$ weeks gestation performed in Victoria in 2019, including data on maternal age, indication for testing, type of procedure, gestational age, and test results. The total count of chromosome abnormalities detected after NIPT were analyzed and the PPV was calculated for each NIPT indication. Results: A total of 1613 women underwent prenatal diagnosis $<25$ weeks overall, of which $419(26.0 \%)$ had testing after receiving an increased chance NIPT result $(n=406)$ or 'no call' result $(n=13)$. The most common conditions suspected on NIPT were trisomy 21,18 or $13(n=224)$ and sex chromosome conditions $(n=82)$. Other NIPT indications included suspected microdeletion syndrome $(n=20)$, rare autosomal aneuploidy $(n=39)$, segmental aneuploidy $(n=21)$ and triploidy $(n=2)$. Twenty-seven unexpected abnormalities were found after an increased chance NIPT result. The PPVs were highest for trisomy 21 (90\%) Klinefelter syndrome (79\%) and trisomy 18 (76\%). The PPV for microdeletion syndromes was 5\%. Suspected rare autosomal trisomies were confirmed in mosaic form in $15 \%$. Conclusion: The range of conditions for which prenatal diagnosis 
is performed after NIPT continues to evolve in Victoria, producing highly variable PPVs and revealing unexpected abnormalities. This raises significant challenges for the provision of maternity care.

\section{The Importance of Ultrasound Prior to Cell-Free DNA Screening for Fetal Chromosomal Abnormalities}

Imogen Brown ${ }^{1,3}$, Melody Menezes ${ }^{1,2}$, Shavi Fernando ${ }^{3,5}$, Fabricio da Silva Costa ${ }^{3,4}$, Jayshree Ramkrishna ${ }^{1}$, Simon Meagher ${ }^{1,2}$ and Daniel Rolnik ${ }^{3,5}$

${ }^{1}$ Monash Ultrasound for Women, Monash IVF Group, Melbourne, Australia, ${ }^{2}$ The University of Melbourne, Melbourne, Australia, ${ }^{3}$ Monash University, Melbourne, Australia, ${ }^{4}$ University of Sao Paulo, Ribeirao Preto, Brazil and ${ }^{5}$ Monash Health, Melbourne, Australia

Background: Cell-free DNA (cfDNA) screening is increasingly becoming utilized by pregnant women in Australasia. Many international bodies recommend that women have an ultrasound performed prior to cfDNA screening however, there is a lack of evidence to support this practice in the general obstetric population. This study aims to determine the incidence of ultrasound findings that have the potential to change clinical management on the day of blood-sampling for cfDNA screening. Methods: A retrospective study was conducted at a tertiary provider of obstetric and gynecological ultrasound in Melbourne, Australia. Individual patient files were reviewed and results were collated for maternal characteristics, pre-cfDNA ultrasound reports, results and test characteristics of both cfDNA screening and diagnostic testing, and genetic counseling notes. The primary outcome was a potential change in patient management due to findings detected on the pre-cfDNA ultrasound. Results: Of 6250 pre-cfDNA ultrasounds, 6207 were included in analysis. Of these, 598 (9.6\%) pregnancies had a finding on precfDNA ultrasound that had the potential to change management. The reasons for this potential change in management were: detection of gestational age below 10 weeks $(245,3.9 \%)$, miscarriage (175, $2.8 \%)$, demised twin $(43,0.7 \%)$, fetal edema $(115,1.9 \%)$ and major structural abnormalities (20,0.3\%). These findings were more common in patients of advanced maternal age and in spontaneous conceptions. Conclusions: An ultrasound prior to cfDNA screening has the potential to change clinical management in almost one in 10 women. The proportion is higher in older age groups and lower in IVF-conceived pregnancies.

\section{Population-Based Analysis of Atypical Chromosomal Abnormalities According to Nuchal Translucency Measurement: An Individual Record Linkage Study of 81,244 Women}

Cecilia Pynaker $^{1}$, Lisa Hui ${ }^{1,2,3,21}$, Anthea Lindquist ${ }^{1,2}$, Alice Poulton ${ }^{1}$, Eliza Kluckow ${ }^{1,3}$, Briohny Hutchinson ${ }^{2}$, Leonard Bonacquisto ${ }^{4}$, Fiona Norris ${ }^{4}$, Mark D. Pertile $e^{4,5}$ Lucy Gugasyan ${ }^{6}$, James Harraway ${ }^{7}$, Amanda Howden ${ }^{8}$, Richard McCoy ${ }^{9}$, Fabricio Da Silva Costa ${ }^{10,11}$, Melody Menezes ${ }^{5,12}$, Ricardo Palma-Dias ${ }^{3,13,14}$, Debbie Nisbet ${ }^{1,14,15}$, Nicole Martin ${ }^{16}$, Michael Bethune ${ }^{1,18}$, Zeffie Poulakis ${ }^{5,19,20}$ and Jane Halliday ${ }^{1,5}$
${ }^{1}$ Reproductive Epidemiology group, Murdoch Children's Research Institute, Melbourne, Australia, ${ }^{2}$ Department of Obstetrics, Mercy Hospital for Women, Melbourne, Australia, ${ }^{3}$ Department of Obstetrics and Gynaecology, University of Melbourne, Melbourne, Australia, ${ }^{4}$ Victorian Clinical Genetics Services, Murdoch Children's Research Institute, Melbourne, Australia, ${ }^{5}$ Department of Paediatrics, University of Melbourne, Melbourne, Australia, ${ }^{6}$ Cytogenetics, Monash Pathology, Monash Medical Centre, Melbourne, Australia, ${ }^{7}$ Sullivan Nicolaides Pathology, Brisbane, Australia, ${ }^{8}$ Cytogenetics, Melbourne Pathology, Melbourne, Australia, ${ }^{9}$ Department of Molecular Genetics, Australian Clinical Labs, Melbourne, Australia, ${ }^{10}$ Department of Gynecology and Obstetrics, Ribeirão Preto Medical School, University of São Paulo, Ribeirão Preto, Brazil, ${ }^{11}$ Department of Obstetrics and Gynaecology, Monash University, Melbourne, Australia, ${ }^{12}$ Monash Ultrasound for Women, Melbourne, Australia, ${ }^{13}$ Women's Ultrasound Melbourne, Melbourne, Australia, ${ }^{14}$ Ultrasound Services, Royal Women's Hospital, Melbourne, Australia, ${ }^{15}$ Department of Medicine and Radiology, University of Melbourne, Melbourne, Australia, ${ }^{16}$ Virtus Diagnostics and Pathology Services, Brisbane, Australia, ${ }^{17}$ Specialist Women's Ultrasound, Melbourne, Australia, ${ }^{18}$ Department of Radiology, University of Melbourne, Melbourne, Australia, ${ }^{19}$ Victorian Infant Hearing Screening Program, Centre for Community Child Health, Royal Children's Hospital, Melbourne, Australia, ${ }^{20}$ Prevention Innovation Group, Population Health, Murdoch Children's Research Institute, Melbourne, Australia and ${ }^{21}$ The Northern Hospital, Melbourne, Australia

Background: In the era of noninvasive prenatal testing (NIPT) the appropriate nuchal translucency (NT) threshold for offering diagnostic testing with chromosomal microarray (CMA) identification remains debated. Aim: To perform a population-based individual record-linkage study of women undergoing combined first trimester screening (CFTS) and prenatal or postnatal diagnosis in order to analyze the frequency and type of chromosome abnormalities according to NT measurement. Methods: Retrospective study of women resident in Victoria, Australia, who underwent CFTS during 2015-16. CFTS results were matched to prenatal diagnostic procedures and postnatal cytogenetic results from products of conception and infants up to 12 months of age, using probabilistic record linkage. An atypical chromosome abnormality was defined as any major chromosome abnormality, other than whole chromosome aneuploidy involving chromosomes $21,18,13, \mathrm{X}$ and Y. Results: Of the 81,244 singleton pregnancies undergoing CFTS, 534 (0.66\%) had an NT 3.0-3.4 mm, and 491 (0.60\%) had an $\mathrm{NT}>3.5 \mathrm{~mm}$. Of the 1779 pregnancies having diagnostic testing, $89.6 \%$ were performed by CMA and 93 atypical chromosome abnormalities were detected, two in fetuses with NT 3.0-3.4 mm. The frequency of an atypical chromosome abnormality with NT $>3.5 \mathrm{~mm}$ was $4.07 \%(95 \% \mathrm{CI}[2.51,6.22])$, with $3.0-3.4 \mathrm{~mm}$, was $0.37 \%(95 \%$ CI $[0.05,1.35])$ and with $<3.0 \mathrm{~mm}$ was $0.09 \%(95 \% \mathrm{CI}[0.07,0.11])$. Conclusion: As expected, the risk of an atypical chromosome abnormality is highest in fetuses with enlarged NT $>3.5 \mathrm{~mm}$. For fetuses with NT $3.0-3.4 \mathrm{~mm}$, however, the $0.37 \%$ risk of an atypical chromosome abnormality ( 1 in 270) is within the traditional range for offering diagnostic testing. 